\title{
CDO, HAME Copulas and an R Package 'CDO'
}

\author{
Master Thesis submitted
}

to

PROF. DR. OSTAP OKHRIN

PROF. DR. WOLFGANG KARL HÄRDLE

Institute for Statistics and Econometrics

Ladislaus von Bortkiewicz Chair of Statistics

C.A.S.E.- Center for Applied Statistics and Econometrics

\section{Humboldt-Universität zu Berlin}
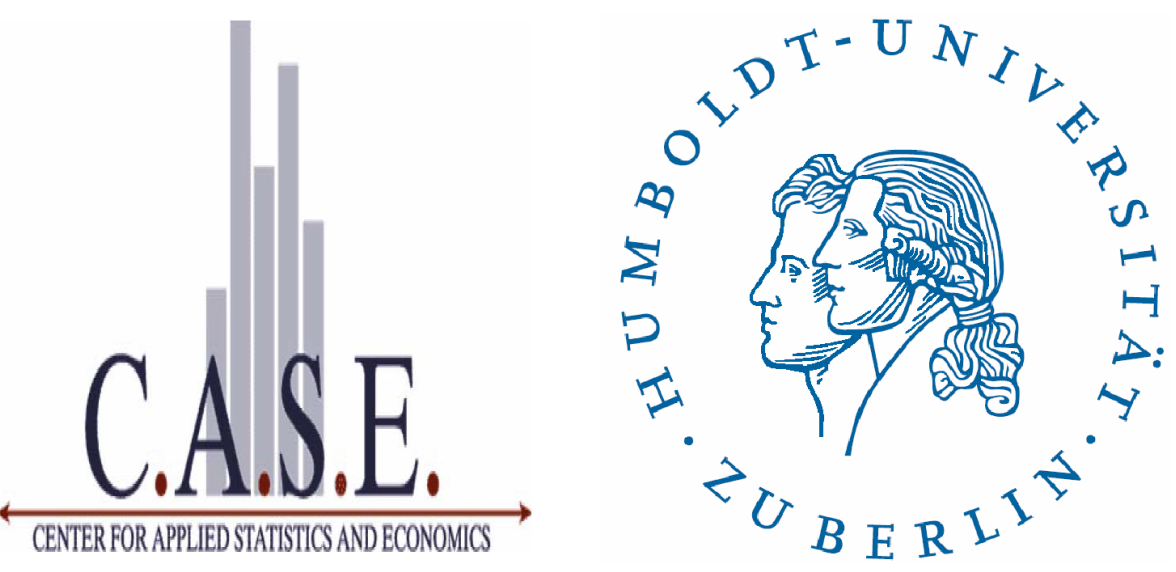

by

\section{Yafei Xu}

(543292)

in partial fulfillment of the requirements

for the degree of

Master of Science in Statistics

Berlin, March 31, 2014 


\section{Acknowledgements}

Foremost, I would like to express my deepest appreciation to my master thesis supervisor PROF. DR. OSTAP OKHRIN, who introduced me to the copulae world and its application in the pricing of the credit derivative, CDO. I thank for his continuous support and help to my study and research, for his supervision, motivation and immense enthusiasm. His introduction and guidance helped me throughout the whole process of my writing of this thesis. Without his encouragement and detailed supervision this thesis would not have been possible.

Also, I would like to thank PROF. DR. WOLFGANG KARL HÄRDLE for his important comments and criticism. He gave me not only suggestions in study but also in life. Especially for his great passion investing in organizing the Sportabzeichen at Berlin Degewo-Stadion, this activity has thoroughly educated me and let me remember that training is everything. After 2 weeks' training in $3 \mathrm{~km}$ running program, my performance has reached 14'14" from no record at first time.

Actually I have accomplished almost all the lectures held by this two professors. Their professionalism, knowledge, vision and dynamic in scientific research always attract more and more students to indulge themselves in the world of statistics. I was just one of them. I treasure the study in the institute for statistics and econometrics as one of the most valuable experiences in my life. At this moment I sincerely wish the both professors all the best and wish the institute for statistics and econometrics a more brilliant future.

Last but not the least, I would like to thank my family, my father Mr. GUIXI XU and mother Mrs. WENHUA ZHANG, whose weekly phone call from Lianyungang to Berlin always brings me spiritual support and always makes me feel the full warm love from the family. Without their selfless engagement and constant support, the accomplishment of the study would have been much more difficult. Dear father and mother, I love you!

Yafei Xu. 


\title{
HUMBOLDT-UNIVERSITÄT ZU BERLIN
}

\author{
Abstract \\ School of Business and Economics \\ Master of Science in Statistics \\ CDO, HAME Copulas and an R Package 'CDO' \\ by Yafei XU
}

\begin{abstract}
After David X. Li's two papers, Li [1] and Li [2], proposing to employ Gaussian copula to the pricing of a collateralized debt obligation (CDO), the copula-based approaches for CDO pricing has been already widely utilized in the finance sector.

In this master thesis we propose a mixed copula approach, i.e. linear combined copula, in pricing the credit default swap (CDS) index tranches (or single tranche CDO (STCDO) termed in Andersen [3]) with up to 3 parameters. Parameters in mixed copula models are calibrated with the market data under numerical optimizations and for the mixed copula models we choose six different component copulas from two families, the elliptical family and the Archimedean family. The mixed copula approach introduced in this master thesis can be utilized to reproduce the spreads of the iTraxx Europe index tranches, i.e. the CDS index based tranches.
\end{abstract}

Three main contributions have been accomplished in this master thesis. Firstly, we introduce mixed copulas to the CDS index tranches pricing as the mixed copula can model the dependence structure of the default times by simultaneously considering the heterogeneity of dependence between sectors and the asymmetrical tail-dependence features. Secondly, we compared the performance of 43 different copula-based models in CDS index tranches pricing, including 3 hierarchical Archimedean copulas, 5 Archimedean copulas, 21 mixed copulas and 14 elliptical copulas, which can be abbreviated with the every first letter to form the caption HAME copulas. Thirdly, we contribute an R package 'CDO', which has contained overall 43 copula models in Table 5.1 of this thesis.

Keywords: Credit Risk, CDO, Copula, Mixed Copula Model, R Package.

JEL Classification: C15, C87, G12, G21. 


\section{Contents}

Acknowledgements

$\begin{array}{lll}\text { Abstract } & \text { ii }\end{array}$

$\begin{array}{ll}\text { Contents } & \text { iii }\end{array}$

List of Figures $\quad$ v

List of Tables vii

Abbreviations ix

1 Introduction 1

2 HAME Copulas $\quad \mathbf{8}$

2.1 Basic Elements of Copula Theory . . . . . . . . . . . . . . . . . . . . . . . . . . . 9

2.2 Elliptical Copula . . . . . . . . . . . . . . . . . . . . . . . . . . . . . . 15

2.3 Archimedean Copula . . . . . . . . . . . . . . . . . . . . . . . . . . . . . . . . . . . . . . . . .

2.4 Hierarchical Archimedean Copula . . . . . . . . . . . . . . . . . . 23

2.5 Mixed Copula . . . . . . . . . . . . . . . . . . . . . . . 24

3 CDO $\quad 27$

3.1 CDS and Basket Default Swap . . . . . . . . . . . . . . . . . 27

3.2 Synthetic CDO and Cash CDO . . . . . . . . . . . . . . . 29

3.3 CDS Index Tranche . . . . . . . . . . . . . . . . . . . . 31

4 CDO Pricing 33

4.1 Mechanism of CDO Pricing . . . . . . . . . . . . . . 33

4.2 Modeling of Defaults . . . . . . . . . . . . . . 36

5 An Empirical Study $4 \mathbf{4 3}$

5.1 Data and Copula Models . . . . . . . . . . . . . . . . . . 43

5.2 Parameter Calibration . . . . . . . . . . . . . . . 56

5.3 Empirical Results and Analysis . . . . . . . . . . . . . . . . . 57

6 An R Package 'CDO'

6.1 Introduction of the Package 'CDO' . . . . . . . . . . . . . . . 71

6.2 Installation and Usage Examples . . . . . . . . . . . . . . . . . 72 
7 Conclusion

Bibliography 78

Declaration of Authorship 82 


\section{List of Figures}

1.1 Issuance of CDOs. (a) Yearly issuance of CDOs with the data from the Securities Industry and Financial Markets Association. (b) Quarterly issuance of CDOs with the data from the Securities Industry and Financial Markets Association. Q MARyearlyIssuance.R Q MARquarterlyIssuance.R ....................... 5

2.110000 Monte Carlo simulations for 3-dimensional Gaussian copula with an exchangeable correlation matrix. QMARgss.R . . . . . . . . . 16

2.2 The first row is for 2-dimensional scatter plots, whose points are drawn from copulas. The second row is for 2-dimensional contour plots of copula densities. The third row is for 2-dimensional copula density plots. From left to right, the first column is for the Gaussian copula with $\rho_{\mathcal{P}}=0.7$. The second column is for the Student- $t$ copula with $\rho_{\mathcal{P}}=0.7$ and the degree of freedom equal to 3 . And the third column is for the Frank copula with $\rho_{\mathcal{K}}=0.7$. QMARpdfgstfrank.R . . . . . . . . . . . 19

2.3 The first row is for 2-dimensional scatter plots, whose points are drawn from copulas. The second row is for 2-dimensional contour plots of copula densities. The third row is for 2-dimensional copula density plots. From left to right, the first column is for the Clayton copula with $\rho_{\mathcal{P}}=0.7$. The second column is for the Gumbel copula with $\rho_{\mathcal{P}}=0.7$. And the third column is for the Joe copula with $\rho_{\mathcal{K}}=0.7$. QMARpdfclaytongumbeljoe.R

2.4 Tree structures of two hierarchical Archimedean copulas, where the upper tree shows the fully hierarchical Archimedean copula and the lower tree shows the partially hierarchical Archimedean copula under 5-dimensional copula context. . . . . . . . . . . . . . . . 25

3.1 The structure of a credit default swap (CDS), which provides the creditor a single name protection from default. . . . . . . . . . . 28

3.2 The structure of a cash collateralized debt obligation, whose main characteristic is the ownership of the reference assets in the underlying pool.

3.3 The structure of a synthetic collateralized debt obligation, whose main characteristic is the selling of CDS contracts and no ownership of the reference assets. . . . . . . . . . . . . . . 30

3.4 The structure of CDS index tranches, whose main feature is selling the insurance-like policy for protection of the basket references. . . . . . . . 31 
4.1 Implied correlation $\rho_{\mathcal{P}, q}, q \in\{1, \ldots, 5\}$ for 5 tranches of iTraxx Europe Series 8 on 2007-11-02 under the Gaussian copula. QMARcorrsmile.R . 40

4.2 A Correlation Matrix owns 6 Sectors, where $\rho_{s i}, i \in\{1, \ldots, 6\}$ is the correlations of the $i$ th sector and $\rho_{7}$ is used to specify the dependence between 7 sectors. . . . . . . . . . . . . . . . . 40

4.3 Copula density plots for 2-dimensional copulas with single parameters. From $(a)-(f)$ : (a) Gaussian copula, (b) Student- $t$ copula, (c) Frank copula, $(d)$ Clayton copula, (e) Gumbel copula, $(f)$ Joe copula. For elliptical copulas the $\rho_{\mathcal{P}}=0.7$ and for Archimedean copulas the $\rho_{\mathcal{K}}=0.7$.

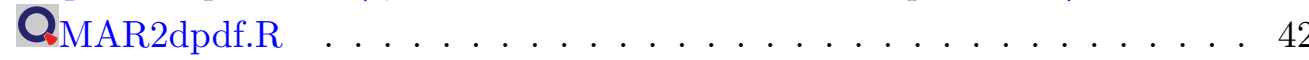

5.1 Tranche spreads at 12 pricing dates of Markit iTraxx Europe Index Series 8. (a) Tranche spreads for four tranches $(q=2,3,4,5)$ of Markit iTraxx Europe Index Series 8 from 20071023 to 20080701 by the Bloomberg Terminal. (b) Tranche spreads for equity tranche of Markit iTraxx Europe Index Series 8 from 20071023 to 20080701 by the Bloomberg Terminal.

Q MARtranchedata1.R Q MARtranchedata2.R . . . . . . . . . . . . 44

5.2 The structure of the correlation matrix $(a) \rho_{g s 1}$ is utilized in Model 3 and Model 38. And the structure of the correlation matrix $(b) \rho_{g s 2}$ is utilized in Model 4 and Model 39. . . . . . . . . . . . . . . . . . . . 48

5.3 The structure of the correlation matrix $(a) \rho_{g s 3}$ is utilized in Model 5 and Model 40. And the structure of the correlation matrix $(b) \rho_{g s 4}$ is utilized in Model 6 and Model 41. . . . . . . . . . . . . . . . . . . . . . 49

5.4 The structure of the correlation matrix $(a) \rho_{g s 5}$ is utilized in Model 7 and Model 42. And the structure of the correlation matrix $(b) \rho_{g s 6}$ is utilized in Model 8 and Model 43. . . . . . . . . . . . . . . . 50

5.5 Tree structure plots for Model 13 in (a), Model 14 in (b), Model 15 in (c) and Model 16 in $(d) \ldots \ldots \ldots \ldots . \ldots \ldots . \ldots \ldots$

5.6 Comparison of models based on the relative difference measures (RDM) in Formula (5.25). (a) The best 3 models and the worst 3 models together in one graph based on the mean of 12 RDMs. (b) The RDM comparison of 43 models under 12 computation dates. QMARbest3worst3.R QMaRrdmcomparison.R . . . . . . . . . . . . . . 60

5.7 Comparison of models based on the relative difference measures (RDM) in Formula (5.25). (a) The best 5 models based on the mean of 12 RDMs. (b) The worst 5 models based on the mean of 12 RDMs. QMARbest5.R

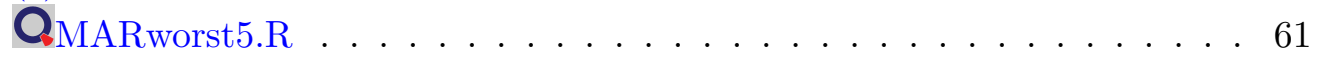

5.8 3-dimensional illustrations of the measure RDMs of 43 models at 12 computation dates. QMaRrdm3d.R QMARrdm3drotate.R . . . . . . . . 62 


\section{List of Tables}

1.1 Literatures of different copula models employed for construction of defaults dependence structure. . . . . . . . . . . . . . 3

1.2 Weights distribution of different sectors taking the iTraxx Europe Series 20 as an example, c.f. Markit [28]. . . . . . . . . . . . . . .

2.1 Five Archimedean copula functions including the Ali-Mikhail-Haq copula, the Frank copula, the Clayton copula, the Gumbel copula and the Joe copula, generator functions and parameters. . . . . . . . . . . . . . 21

3.1 Spreads of tranches of the iTraxx Europe Series 8 on 2007-10-23 with maturities of 5 years and 6 years sourced from the Bloomberg Terminal.

5.1 Abbreviations: gs: Gaussian, $t$ : Student- $t, f$ : Frank, $c$ : Clayton, $g$ : Gumbel, $j$ : Joe, gsi, $i \in\{1, \ldots, 6\}$ : Gaussian with the correlation matrix $\rho_{g s i}, i \in\{1, \ldots, 6\}, t j, j \in\{38, \ldots, 43\}$ : Student- $t$ with the same correlation matrix structure as $\rho_{g s i}, i \in\{1, \ldots, 6\}, n g$ : hierarchical Archimedean copula with the Gumbel generator function. Hofert (2008), Choros-Tomczyk, Härdle, Okhrin (2013). . . . . . . . . . . . . . . . 56

5.2 Measure $R D M$ after the calibration for copula models from Model 1 to Model 22, $M=10^{4}$. . . . . . . . . . . . . . . . 63

5.3 Measure $R D M$ after the calibration for copula models from Model 23 to Model 43, $M=10^{4}$. . . . . . . . . . . . . . . . . 64

5.4 Calibrated parameters for Model 1 to Model 12 under the optimization with Formula (5.25) and the Monte Carlo simulation $M=10^{4}$. . . . . 65

5.5 Calibrated parameters for Model 13 to Model 20 under the optimization with Formula (5.25) and the Monte Carlo simulation $M=10^{4}$. . . . . . 66

5.6 Calibrated parameters for Model 21 to Model 27 under the optimization with Formula (5.25) and the Monte Carlo simulation $M=10^{4}$. . . . . 67

5.7 Calibrated parameters for Model 28 to Model 34 under the optimization with Formula (5.25) and the Monte Carlo simulation $M=10^{4}$. . . . . 68

5.8 Calibrated parameters for Model 35 to Model 43 under the optimization with Formula (5.25) and the Monte Carlo simulation $M=10^{4}$. . . . . 69

5.9 The ranking of 43 models under the mean RDM. Abbreviations: gs: Gaussian, $t$ : Student- $t, f$ : Frank, $c$ : Clayton, $g$ : Gumbel, $j:$ Joe, gsi, $i \in$ $\{1, \ldots, 6\}$ : Gaussian with the correlation matrix $\rho_{g s i}, i \in\{1, \ldots, 6\}$, $t j, j \in\{38, \ldots, 43\}$ : Student- $t$ with the same correlation matrix structure as $\rho_{g s i}, i \in\{1, \ldots, 6\}, n g$ : hierarchical Archimedean copula with the Gumbel generator function. . . . . . . . . . . . . . . . . . . . . 70 
6.1 List of employed copula models. Abbreviations: gs: Gaussian, $t$ : Student$t, f$ : Frank, $c$ : Clayton, $g$ : Gumbel, $j$ : Joe, gsi, $i \in\{1, \ldots, 6\}$ : Gaussian with the correlation matrix $\rho_{g s i}, i \in\{1, \ldots, 6\}, t j, j \in\{38, \ldots, 43\}$ : Student- $t$ using the correlation matrix structure $\rho_{g s i}, i \in\{1, \ldots, 6\}, n g$ : hierarchical Archimedean copula with the Gumbel generator function. . . 72 


\title{
Abbreviations
}

\author{
ABS Asset-Backed Security \\ AC Archimedean Copula \\ CBO Collateralized Bond Obligation \\ CDF Cumulative Distribution Function \\ CDO Collateralized Debt Obligation \\ CDS Credit Default Swap \\ CDX Credit Default IndeX \\ CLO Collateralized Loan Obligation \\ HAC Hierarchical Archimedean Copula \\ HAME Hierarchical Archimedean Copula \\ Archimedean Copula \\ Mixed Copula \\ Elliptical Copula \\ LGD Loss Given Default \\ NAC Nested Archimedean Copula \\ PDF Probability Density Function \\ RDM Relative Difference Measure \\ SPV Special Purpose Vehicle \\ STCDO Single Tranche Collateralized Debt Obligation
}




\section{Chapter 1}

\section{Introduction}

In recent years, the financial innovation has accelerated significantly with the introduction of a lot of new contracts and standard products. In credit derivative market new vehicles including credit default swap (CDS), basket default swap, credit default swap index (CDX), collateralized debt obligation (CDO), and credit default swap index tranches (CDS index tranches) have attracted more and more attention. Especially for the product $\mathrm{CDO}$, from one perspective, it provides credit investors a new opportunity to diversify their credit portfolio's risk, as a CDO can provide creditors not only a single name protection, which can be fulfilled by a CDS contract, but also a multi-name protection for the credit portfolios by employing a slicing technique termed as "tranche" under a large pool of debtors. The CDO contract is different from the basket default swap, which is only based on the $n$-th default of the underlying assets pool, while a tranche in a CDO focuses on a total loss given default (LGD) of the underlying pool with a settled interval between an attachment point and a detachment point. From another perspective, the complex mechanism of the CDO contract brings investors problems in the accurate pricing, which includes three main questions: modeling the dependence of default times, i.e. a joint cumulative distribution function (CDF) of default times, modeling the marginal distribution function of a single name default time, which is equivalent to model a survival function of a single default time, and modeling of the loss given default (LGD).

In the studies of the CDO pricing there can be basically diverged into two directions. The first direction is the factor copula model, which is originated from two papers by 
Vasicek [4] and Vasicek [5], which propose to use a latent variable represented as a sum of two factors including a common factor or market factor and an idiosyncratic factor, whose idea can be traced from Merton [6]. The main point of this model is to construct an unconditional CDF of the overall portfolio loss instead of direct modeling of the CDF of multi-name default times, with which later the portfolio loss expectation can be incorporated for the tranche pricing. In the literatures this model is often called the one-factor Gaussian copula model. The second direction is the Monte Carlo based copula model proposed partly by $\mathrm{Li}$ [1] and the whole theory has been published in $\mathrm{Li}$ [2]. Li's approach focuses on modeling the multi-name default times with a high dimensional exchangeable Gaussian copula combined with a transform of the single-name survival function. Compared with Vasicek [4] and Vasicek [5], Li [2] model construct the defaults dependence structure directly on the default times. In Frey and McNeil [7] both models are concluded together as the latent variable model, in which the latent variable in Vasicek [4] and Vasicek [5] is the asset value and in $\mathrm{Li}$ [2] is the default time. As both models choose a high dimensional exchangeable Gaussian copula, therefore drawbacks in the Gaussian copula exist also in both models including lacking the heterogeneity of dependence between sectors, the asymmetrical tail-dependence, with which the exchangeable Gaussian copula based pricing is not accurate compared with market spreads of CDO tranches.

As the existence of the problems of the drawbacks of the Gaussian copula model referred above, many new methods have been proposed for specifying the defaults dependence structure by choosing new copulas which possess partly or whole features such as the heterogeneity of dependence in sectors and the asymmetrical tail-dependence. In choosing new copulas direction literatures are abundant, such as the multi-parameter Gaussian copula model in Hofert and Scherer [8], Hofert [9] and Choros-Tomczyk et al. [10], the Student- $t$ copula model in Lindskog and McNeil [11], Embrechts et al. [12], Frey and McNeil [7], Andersen et al. [13], Greenberg et al. [14], Mashal et al. [15], Demarta and McNeil [16] and Schloegl and O'Kane [17], the Clayton copula model in Schoenbucher and Schubert [18], Lindskog and McNeil [11], Schoenbucher [19], Rogge and Schoenbucher [20], , Gregory and Laurent [21], Gregory and Laurent [22] and Friend and Rogge [23], the Marshall Olkin copula model in Lindskog and McNeil [11], Duffie and Singleton [24] and Giesecke [25], the double- $t$ copula model in Hull and White [26], the 


\begin{tabular}{l|l}
\hline \hline Model & Literature \\
\hline Multi-Parameter Gaussian Copula Model & Hofert and Scherer [8], Hofert [9],Choros-Tomczyk et al. [10] \\
Student- $t$ Copula Model & Lindskog and McNeil [11], Embrechts et al. [12] \\
& Frey and McNeil [7], Andersen et al. [13] \\
& Greenberg et al. [14], Mashal et al. [15] \\
& Demarta and McNeil [16], Schloegl and O'Kane [17] \\
& Schoenbucher and Schubert [18], Lindskog and McNeil [11] \\
& Schoenbucher [19], Rogge and Schoenbucher [20] \\
& Gregory and Laurent [21], Gregory and Laurent [22] \\
& Friend and Rogge [23] \\
& Lindskog and McNeil [11], Duffie and Singleton [24] \\
Marshall Olkin Copula Model & Giesecke [25] \\
& Hull and White [26] \\
Double- $t$ Copula Model & Hofert and Scherer [8], Hofert [9], Choros-Tomczyk et al. [10] \\
\hline \hline
\end{tabular}

TABLE 1.1: Literatures of different copula models employed for construction of defaults dependence structure.

hierarchical Archimedean copula model in Hofert and Scherer [8], Hofert [9] and ChorosTomczyk et al. [10]. Following we list a Table 1.1 to give a literature overview of the above referred copula-based models.

In this master thesis we mainly focus on the CDO pricing approach based on the mixed copula model, i.e. the linear combined copula model, which can be seen as a generalization of copula models employed in CDO pricing. Mixed copula can join different copula families together so that the virtues of different copulas can be utilized for default dependence structure construction. A vanilla example is to join a Gaussian copula and a Student- $t$ copula linearly, which can overcome the drawback of an exchangeable Gaussian copula lacking the tail-dependence which appears frequently in financial time series, by increasing the weight of Student- $t$ copula in the new combined model. Another example is to combine two Archimedean copulas linearly together to grant the new copula model with feature of the asymmetrical tail-dependence, especially the right tail-dependence such as the Joe and Gumbel copulas. According to the Monte Carlo simulation the mixed copula method can be conveniently incorporated in the evaluation of a CDO product.

Figure $1.1(a)$ and (b) show individually the yearly issuance of CDOs and the quarterly issuance of CDOs in the world. Obviously we can see from the figure of the yearly issuance of CDOs that after the financial crisis from 2007 the issuance of CDOs has already sharply decreased and the issuance at the end of 2013 is almost the same as it in 2001, but from Childs [27] and Figure 1.1 (b) we see that after the bottom volume 


\begin{tabular}{l|c}
\hline \hline Sector & Weight \\
\hline Autos and Industrials & $24 \%$ \\
Consumers & $24 \%$ \\
Energy & $16 \%$ \\
Financials & $20 \%$ \\
Technology, Media and Telecommunications & $16 \%$ \\
\hline \hline
\end{tabular}

TABLE 1.2: Weights distribution of different sectors taking the iTraxx Europe Series 20 as an example, c.f. Markit [28].

happened in the 3rd quarter in 2009 CDOs' issuance booms again quarterly and reached a peak at the 1st quarter in 2013, which means that although CDOs have caused huge losses for credit investors in financial crisis, yet CDOs are still attractive vehicles for investment, especially for risk management in credit risk hedging, therefore the study of more accurate CDO pricing is worthwhile.

In the empirical study of this master thesis we employ the data set of the iTraxx Europe Index Series 8 managed by the company Markit from the Bloomberg terminal. iTraxx Europe is a CDS index containing 125 names dispersed in 6 diverse sectors including the autos sector, the industrials sector, the consumers sector, the energy sector, the financial sector and the technology, media and telecommunications sector (TMT sector). According to the latest iTraxx Europe Series 20 the weights of the 6 sectors is listed in Table 1.2 (c.f. Markit [28]). The data that we utilized in this master thesis is the iTraxx Europe Series 8 with 6 sectors and overall 125 entities containing 10 names in the autos sector, 20 names in the industrials sector, 30 names in the consumers sector, 20 names in the energy sector, 25 names in the financial sector and 20 names in the TMT sector.

In the CDO pricing part we construct mixed copula based models and in every mixed copula model we incorporate 2 component copulas from 2 families, the elliptical family including the Gaussian copula and the Student- $t$ copula and the Archimedean family including the Frank, the Clayton, the Gumbel and the Joe copula, therefore we input totally 21 mixed copula models, which can overcome drawbacks such as the heterogeneity of dependence between sectors and the asymmetrical tail-dependence existed in the exchangeable Gaussian copula model for the construction of defaults dependence. In the modeling of a mixed copula with 2 component copulas, we calibrate the model up to 3 parameters, i.e. every mixed copula model includes two component copulas. 


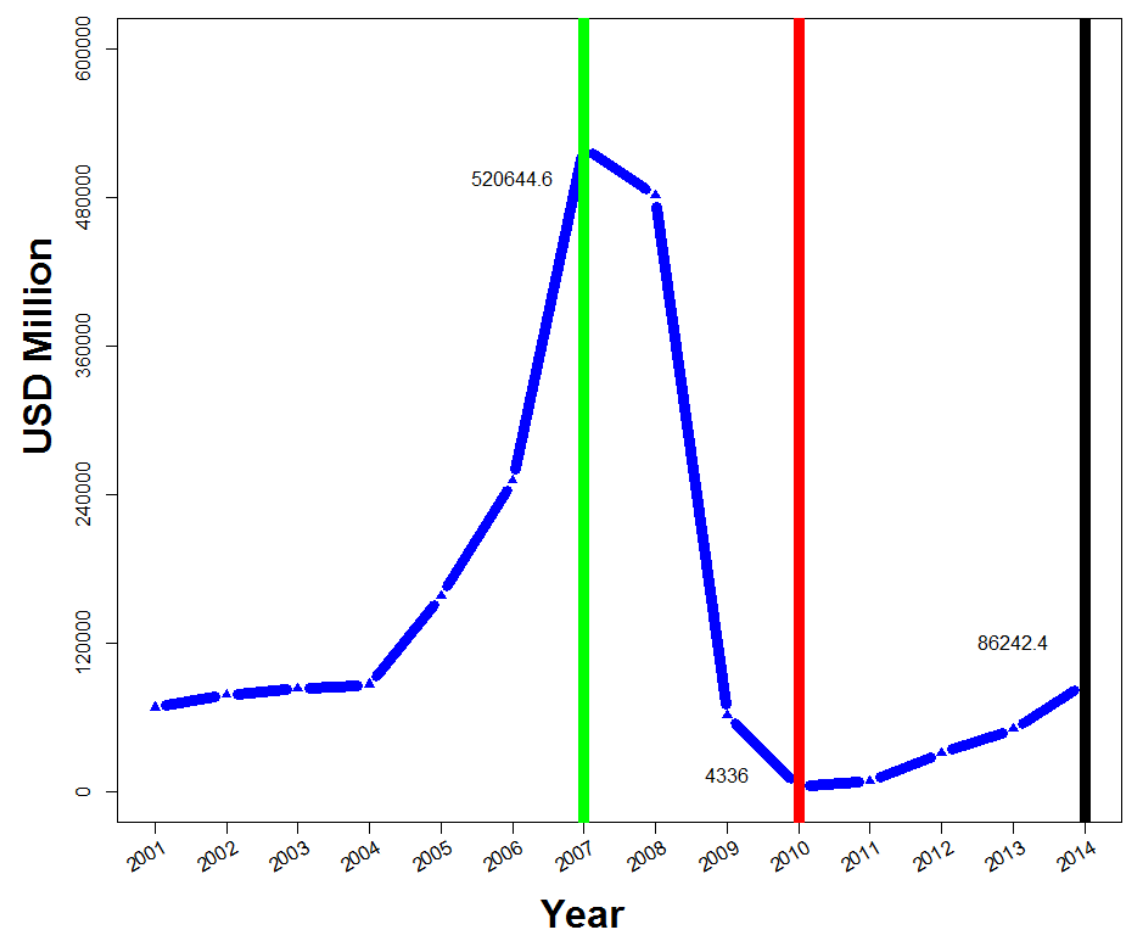

(a)

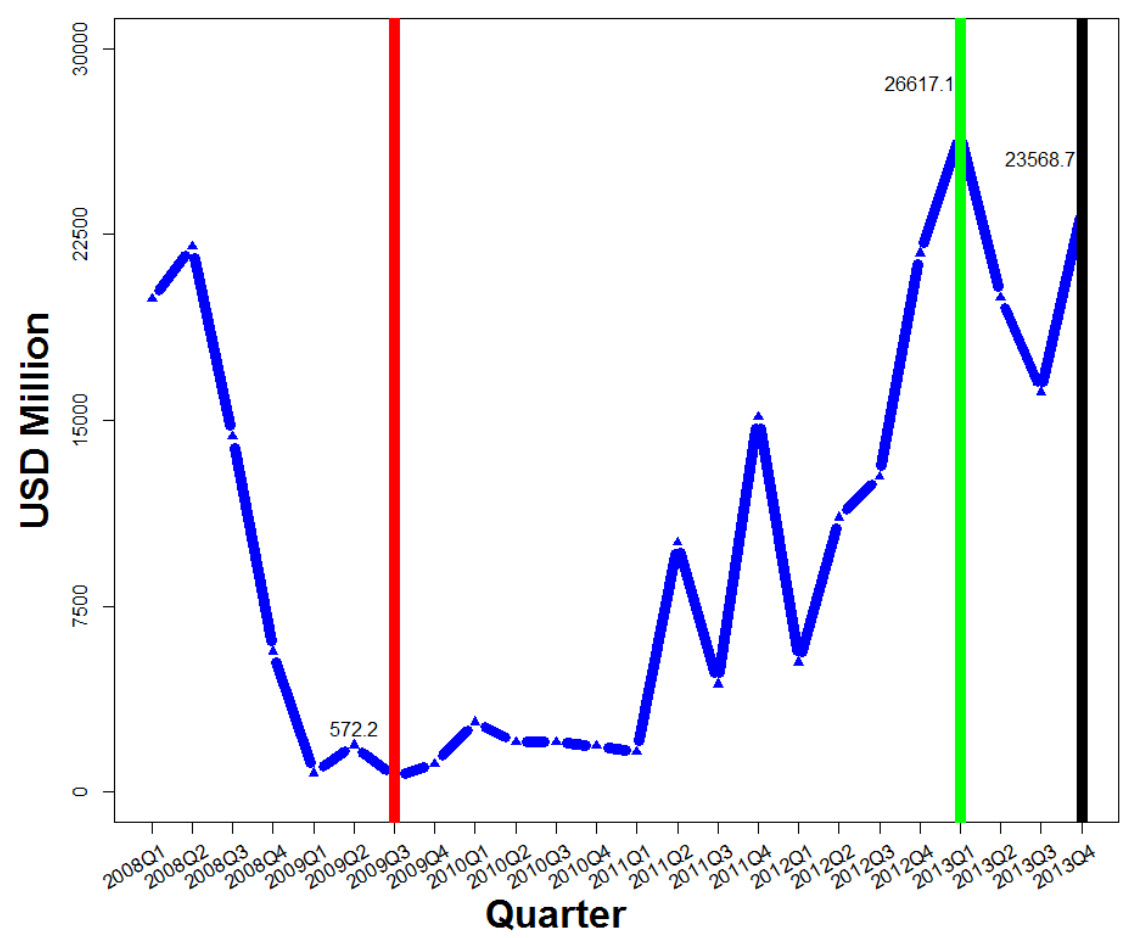

(b)

Figure 1.1: Issuance of CDOs. (a) Yearly issuance of CDOs with the data from the Securities Industry and Financial Markets Association. (b) Quarterly issuance of CDOs with the data from the Securities Industry and Financial Markets Association. Q. MARyearlyIssuance.R O MARquarterlyIssuance.R 
Although in this master thesis we only give the one by one mixed copula model, which let every component copula have a single parameter, yet with more powerful computation capacity this mixed copula model can be generalized to more than 2 component copulas and containing more flexible type of copulas such as hierarchical Archimedean copulas to strengthen the heterogeneity feature in specifying the defaults dependence structure.

The main purpose of this master thesis is to employ the mixed copula models in reproduction of the spreads of CDO tranches. We calibrate the parameters in the mixed copula models with numerical optimizations, whose objective function is a function of the difference obtaining from the mixed copula model based theoretical spreads and the real market spreads. All parameters are computed by minimizing the above referred objective function. In this master thesis we evaluate overall 5 tranches consistent with the Bloomberg quoting convention.

We accomplish in this master thesis in employing 21 mixed copula models into the CDO pricing and we compare also these 21 mixed copula models with the other 22 copula models including 6 single parameter copula models, containing Gaussian, Student- $t$, Frank, Clayton, Gumbel and Joe copulas, and 4 hierarchical archimedean copulas ever appeared in Choros-Tomczyk et al. [10] and Hofert [9] with the Gumbel copula as the generator with up to 3 parameters, 6 multi-parameter Gaussian copula models, and 6 multi-parameter Student- $t$ copula models are also included. Totally 43 models are employed in this master thesis for reproducing the spreads of CDO tranches and after comparison of the performance in pricing we give a ranking of these 43 models based on the relative difference measure and we find the mixed copula models rank in the top place.

This master thesis is structured in four parts. In Chapter 2 we give a introduction of the HAME copulas, i.e. four families of copulas including the elliptical family , the Archimedean family, the hierarchical Archimedean family and the mixed copula family. In Chapter 3 we introduce the CDO and its related products. The theory of CDO pricing is given in Chapter 4. In Chapter 5 we perform the empirical study including the spreads computation and the parameter calibration in CDO pricing and analyze the empirical results. In Chapter 6 we give an introduction to the $\mathrm{R}$ package "CDO", which is a part of this master thesis and can be already downloaded from 
https://sites.google.com/site/cdowithr/. We conclude the master thesis in Chapter 7. After the bibliography of this master thesis we have affiliated a manual of the $R$ package "CDO" for reference. 


\section{Chapter 2}

\section{HAME Copulas}

Copula is a word in Latin as a noun which means a "link" or a "band" used to connect two things together. In statistical sense copula is a function which joints or couples the marginal CDFs to form a multivariate CDF. The earliest literature describing features of such function can be originated to Wassilij Hoeffding in Hoeffding [29], and Abe Sklar used at the first time the word copula in Sklar [30], where he gave the Sklar's theorem. Nowadays the copula function has been utilized widely in geology, engineering, finance, economics and other disciplines. Especially in quantitative risk management, copula applications appear in more and more studies, whose reasons can be concluded in two parts: firstly copula functions can be used for construction of the dependence of default times which let us extend the classical Gaussian world to a new horizon which can integrate more virtues in specifying the financial dynamics, and secondly copulas can separate the dependence construction independently into choosing a joint $\mathrm{CDF}$ and margins, which grants people more flexibility and convenience in modeling and simulation.

This chapter is called the HAME Copulas for our introduction of the hierarchical Archimedean copula, the Archimedean copula, the mixed copula and the elliptical copula, i.e. HAME is the abbreviation of these four copula families. In this chapter we give five sections. In section 1 we give the basics of copulas. Then we introduce three copula families used frequently in finance containing the the elliptical family, the Archimedean family and the hierarchical Archimedean family. We also introduce the mixed family in the last section, which is employed in this master thesis for CDO pricing. As this 
chapter is only a brief overview of copulas, therefore for details of copula theory we refer to Joe [31] and Nelsen [32] and for copula application in finance we refer to Bouye [33].

\subsection{Basic Elements of Copula Theory}

In this section we introduce definitions and related theorem of copula functions. Firstly we give the definition of the copula.

Definition 2.1 (Copula). A $K$-dimensional copula is a function $C:[0,1]^{K} \rightarrow[0,1]$ with the following properties:

1. $C\left(u_{1}, u_{2}, \ldots, u_{K}\right)=0$, if at least one $u_{k}=0, k \in\{1, \ldots, K\}$.

2. $C\left(1, \ldots, 1, u_{k}, 1, \ldots, 1\right)=u_{k}, \forall u_{k} \in[0,1], k \in\{1, \ldots, K\}$.

3. $\forall u=\left(u_{1}, u_{2}, \ldots, u_{K}\right), v=\left(v_{1}, v_{2}, \ldots, v_{K}\right), a=\left(a_{1}, a_{2}, \ldots, a_{K}\right) \in[0,1]^{K}$ with $u_{k} \leq$ $v_{k}, k \in\{1, \ldots, K\}$ such that,

$$
\sum_{a \in \nu} \operatorname{Ind}(a) C(a) \geq 0
$$

where

$$
\begin{gathered}
\nu=\left\{a \in[0,1]^{K} \mid a_{k} \in\left\{v_{k}, u_{k}\right\}, \forall k \in\{1, \ldots, K\}\right\}, \\
\operatorname{Ind}(a)= \begin{cases}1 & \text { if } \sum_{k=1}^{K} d\left(a_{k}\right) \text { is an even number, } \\
-1 & \text { if } \sum_{k=1}^{K} d\left(a_{k}\right) \text { is an odd number, }\end{cases} \\
d\left(a_{k}\right)= \begin{cases}1 & \text { if } a_{k}=u_{k}, k \in\{1, \ldots, K\}, \\
2 & \text { if } a_{k}=v_{k}, k \in\{1, \ldots, K\} .\end{cases}
\end{gathered}
$$

From the above definition we can conclude that the copula is a grounded and $K$ increasing function defined in a hypercube $[0,1]^{K}$ with a range in a unit interval $[0,1]$ which is similar to a $K$-dimensional $\mathrm{CDF}$, meanwhile the copula function also owns margins which are also defined in a unit interval $[0,1]$. 
Copula has a property that under the strictly monotone transformation of a random vector $\left(X_{1}, \ldots, X_{K}\right)^{\top}$ the copula is invariant, which can be given in the following theorem.

Theorem 2.2. Let $C$ be the copula of a continuous random vector $\left(X_{1}, \ldots, X_{K}\right)^{\top} \in \mathbb{R}^{K}$ and $\gamma_{1}, \ldots, \gamma_{K}$ are strictly increasing functions such that $\gamma_{k}: \mathbb{R} \rightarrow \mathbb{R}, \in\{1, \ldots, K\}$, then the vector $\left(\gamma_{1}\left(X_{1}\right), \ldots, \gamma_{K}\left(X_{K}\right)\right)^{\top}$ has the same copula $C$ as the vector $\left(X_{1}, \ldots, X_{K}\right)^{\top}$.

\section{Sklar's Theorem}

A pivotal usage of copula is that it can be used as an instrument to construct a joint CDF of $K$ random variables by choosing independently a copula function and $K$ marginal CDFs, which means coupling margins. This feature can be derived from the Sklar's Theorem as follows.

Theorem 2.3 (Sklar's Theorem). Given a K-dimensional joint distribution function $F$ such that $F\left(x_{1}, \ldots, x_{K}\right)=\mathbb{P}\left(X_{1} \leq x_{1}, \ldots, X_{K} \leq x_{K}\right)$ of a random vector $\left(X_{1}, X_{2}, \ldots\right.$, $\left.X_{K}\right)^{\top}$ with margins $F_{k}(x)=\mathbb{P}\left(X_{k} \leq x\right)$, there exists a $K$-dimensional copula $C$ such that

$$
F\left(x_{1}, \ldots, x_{K}\right)=C\left[F_{1}\left(x_{1}\right), \ldots, F_{K}\left(x_{K}\right)\right] .
$$

The copula $C$ is unique if $F_{k}, k \in\{1,2, \ldots, K\}$, is continuous, otherwise $C$ is uniquely defined on $\prod_{i=k}^{K}$ Range $\left(F_{k}\right)$. Conversely, if all $F_{k}, k \in\{1,2, \ldots, K\}$ are 1-dimensional distribution function and $C$ is a $K$-dimensional copula then the function $F\left(x_{1}, \ldots, x_{K}\right)$ is a $K$-dimensional distribution function.

From the Sklar's theorem we obtain two important formulas,

$$
\begin{aligned}
& F\left(x_{1}, \ldots, x_{K}\right)=C\left(F_{1}\left(x_{1}\right), \ldots, F_{n}\left(x_{K}\right)\right), \\
& C\left(u_{1}, \ldots, u_{K}\right)=F\left(F_{1}^{-1}\left(u_{1}\right), \ldots, F_{K}^{-1}\left(u_{K}\right)\right) .
\end{aligned}
$$

The Formula (2.6) means that a $K$-dimensional joint CDF can be decomposed into two parts, a copula function and $K$ marginal CDFs, which also means we can construct a flexible joint $\mathrm{CDF}$ of $K$ random variables $\left(X_{1}, X_{2}, \ldots, X_{K}\right)^{\top}$ by giving a dependence structure, i.e. a copula function and marginal CDFs. And the Formula (2.7) tells us 
a copula function $C$ can be constructed by a $K$-dimensional joint CDF and inverse marginal CDFs, which can be used conveniently for drawing samples of a random vector $\left(U_{1}, U_{2}, \ldots, U_{K}\right)^{\top}$ under the Monte Carlo simulation, in which we can draw samples of a random vector $\left(X_{1}, X_{2}, \ldots, X_{K}\right)^{\top}$ firstly from the joint $\operatorname{CDF} F\left(x_{1}, \ldots, x_{K}\right)$ and then obtain $\left(U_{1}, U_{2}, \ldots, U_{K}\right)^{\top}$ by a transformation such that $\left(F_{1}\left(X_{1}\right), \ldots, F_{K}\left(X_{K}\right)\right)^{\top}$. We will give examples of simulation later in this chapter.

\section{Copula Density}

As the definition of a copula shown in Theorem 2.3 that a $K$-copula function is similar to a $K$-dimensional joint $\mathrm{CDF}$ with a domain in a $K$-cube and margins defined in a unit interval $[0,1]$, therefore it is reasonable that a copula function also possesses a density function, i.e. a PDF, which can be represented as follows,

$$
c\left(u_{1}, \ldots, u_{K}\right)=\frac{\partial C\left(u_{1}, \ldots, u_{K}\right)}{\partial u_{1}, \ldots, \partial u_{K}}, \text { where } u_{1}, \ldots, u_{K} \in[0,1]
$$

Analogously, we can represent the PDF of $F\left(x_{1}, \ldots, x_{K}\right)$ in the form of a copula density with the Formula (2.9) and the Theorem 2.3 such that,

$$
f\left(x_{1}, \ldots, x_{K}\right)=c\left(F_{1}\left(x_{1}\right), \ldots, F_{K}\left(x_{K}\right)\right) \prod_{k=1}^{K} f_{k}\left(x_{k}\right), \text { where } x_{1}, \ldots, x_{K} \in \mathbb{R} .(2.9)
$$

\section{Fréchet-Hoeffding Bounds}

Next we introduce the Fréchet-Hoeffding bounds of a copula function. Firstly we give some notations for the definition. Let $L^{B}:[0,1]^{K} \rightarrow[0,1], U^{B}:[0,1]^{K} \rightarrow[0,1]$ and be represented as follows,

$$
\begin{aligned}
U^{B}(u) & =\min \left(u_{1}, \ldots, u_{K}\right), \\
L^{B}(u) & =\max \left(u_{1}+\cdots+u_{K}-K+1,0\right) .
\end{aligned}
$$

Then the Fréchet-Hoeffding bounds inequality can be shown as follows, 
Theorem 2.4 (Fréchet-Hoeffding Bounds Inequality). Let $C$ be an arbitrary K-dimensional copula, then for every point $u \in[0,1]^{K}$ such that

$$
L^{B}(u) \leq C(u) \leq U^{B}(u)
$$

\section{Dependence Measures of Copulas}

Firstly, a frequently utilized dependence measure is the Pearson's correlation (or linear correlation) defined as follows,

Definition 2.5 (Pearson's Correlation). Let $\left(X_{1}, X_{2}\right)^{\top} \in \mathbb{R}^{2}$ be random vector with restrictions that $\operatorname{Var}\left(X_{k}\right)<\infty, \operatorname{Var}\left(X_{k}\right) \neq 0, k \in\{1,2\}$, then the Pearson's correlation $\rho_{\mathcal{P}}$ of $X_{1}$ and $X_{2}$ can be represented as follows,

$$
\rho_{\mathcal{P}}\left(X_{1}, X_{2}\right)=\frac{\operatorname{Cov}\left(X_{1}, X_{2}\right)}{\sqrt{\operatorname{Var}\left(X_{1}\right)} \sqrt{\operatorname{Var}\left(X_{2}\right)}},
$$

where $\operatorname{Cov}()$ is the covariance operator and $\operatorname{Var}()$ the variance operator.

The linear correlation is an important dependence measure in statistics for its ease in interpretation and computation, especially in elliptical copula family such as the Gaussian copula and the Student- $t$ copula, however it is restricted for those copulas that are not elliptical and also for its second moment condition restrictions that $\operatorname{Var}(X)<\infty$ and $\operatorname{Var}(X) \neq 0$ which even can not be utilized for some cases in the elliptical family such as the Student- $t$ CDF with the degree of freedom equal to 2 having an infinite second moment.

As there exist drawbacks in linear correlation which is not suitable for the dependence measure of non-elliptical copulas then two new measures are introduced, the Kendall's tau $\rho_{\mathcal{T}}$ and the Spearman's rho $\rho_{\mathcal{S}}$.

Definition 2.6 (Kendeall's tau). Let $\left(X_{1}, Y_{1}\right)^{\top},\left(X_{2}, Y_{2}\right)^{\top} \in \mathbb{R}^{2}$ be independent random vectors, then the Kendeall's tau of $X_{1}$ and $Y_{1}$ can be represented as follows,

$$
\rho_{\mathcal{T}}\left(X_{1}, Y_{1}\right)=\mathbb{P}\left[\left(X_{1}-X_{2}\right)\left(Y_{1}-Y_{2}\right)>0\right]-\mathbb{P}\left[\left(X_{1}-X_{2}\right)\left(Y_{1}-Y_{2}\right)<0\right] .
$$

The essence of the Kendall's tau is the difference between the probability of the concordance $\left\{\left(X_{1}-X_{2}\right)\left(Y_{1}-Y_{2}\right)>0\right\}$ and the dis-concordance $\left\{\left(X_{1}-X_{2}\right)\left(Y_{1}-Y_{2}\right)<0\right\}$. 
Theorem 2.7. Let $C$ be an arbitrary copula of a continuous random vector $\left(X_{1}, Y_{1}\right)^{\top} \in$ $\mathbb{R}^{2}$, then the formula for computation of the Kendall's tau is as follows

$$
\rho_{\mathcal{T}}\left(X_{1}, Y_{1}\right)=4 \iint_{[0,1]^{2}} C\left(u_{1}, u_{2}\right) \mathrm{d} C\left(u_{1}, u_{2}\right)-1
$$

Here we can see that the Kendall's tau is a function of the expectation of a bivariate random vector $\left(U_{1}, U_{2}\right)^{\top}$ under a joint $\operatorname{CDF} C\left(u_{1}, u_{2}\right)$ such that $\rho_{\mathcal{T}}\left(X_{1}, Y_{1}\right)=$ $4 \mathbb{E}\left(C\left(U_{1}, U_{2}\right)\right)-1$.

Definition 2.8 (Spearman's rho). Let $\left(X_{1}, Y_{1}\right)^{\top},\left(X_{2}, Y_{2}\right)^{\top}$ and $\left(X_{3}, Y_{3}\right)^{\top} \in \mathbb{R}^{2}$ be independent random vectors, then the Spearman's rho of $X_{1}$ and $Y_{1}$ can be represented as follows,

$$
\rho_{\mathcal{S}}\left(X_{1}, Y_{1}\right)=3\left(\mathbb{P}\left[\left(X_{1}-X_{2}\right)\left(Y_{1}-Y_{3}\right)>0\right]-\mathbb{P}\left[\left(X_{1}-X_{2}\right)\left(Y_{1}-Y_{3}\right)<0\right]\right) .
$$

Theorem 2.9. Let $C$ be an arbitrary copula of a continuous random vector $\left(X_{1}, X_{2}\right)^{\top} \in$ $\mathbb{R}^{2}$, then the formula for computation of the Kendall's tau is as follows

$$
\rho_{\mathcal{S}}\left(X_{1}, Y_{1}\right)=12 \iint_{[0,1]^{2}} C\left(u_{1}, u_{2}\right) \mathrm{d} u_{1} \mathrm{~d} u_{2}-3
$$

We give the three dependence measures together as follows,

$$
\begin{aligned}
\rho_{\mathcal{P}}\left(X_{1}, Y_{1}\right) & =\frac{\operatorname{Cov}\left(X_{1}, X_{2}\right)}{\sqrt{\operatorname{Var}\left(X_{1}\right)} \sqrt{\operatorname{Var}\left(X_{2}\right)}}, \\
\rho_{\mathcal{S}}\left(X_{1}, Y_{1}\right) & =3\left(\mathbb{P}\left[\left(X_{1}-X_{2}\right)\left(Y_{1}-Y_{3}\right)>0\right]-\mathbb{P}\left[\left(X_{1}-X_{2}\right)\left(Y_{1}-Y_{3}\right)<0\right]\right), \\
\rho_{\mathcal{K}}\left(X_{1}, Y_{1}\right) & =\mathbb{P}\left[\left(X_{1}-X_{2}\right)\left(Y_{1}-Y_{2}\right)>0\right]-\mathbb{P}\left[\left(X_{1}-X_{2}\right)\left(Y_{1}-Y_{2}\right)<0\right] .
\end{aligned}
$$

\section{Tail Dependence}

The tail dependent behavior is not rare in the financial time series and for the measuring of the tail dependence we give the definitions for the upper tail dependence and the lower tail dependence.

Definition 2.10 (Upper Tail Dependence). Let $\left(X_{1}, X_{2}\right)^{\top} \in \mathbb{R}^{2}$ be a continuous random vector and $X_{1} \sim F_{1}\left(x_{1}\right), X_{2} \sim F_{2}\left(x_{2}\right)$, if $\lambda_{\text {upper }} \in(0,1]$ then the coefficient of the upper 
tail dependence of $\left(X_{1}, X_{2}\right)^{\top}$ can be represented as follows,

$$
\lambda_{\text {upper }}=\lim _{u \rightarrow 1} \mathbb{P}\left[X_{2}>F_{2}^{-1}(u) \mid X_{1}>F_{1}^{-1}(u)\right]
$$

As $\mathbb{P}\left[X_{2}>F_{2}^{-1}(u) \mid X_{1}>F_{1}^{-1}(u)\right]$ in Formula (2.18) is equivalent to the formulation such that

$$
\frac{1-\mathbb{P}\left[X_{1} \leq F_{1}^{-1}(u)\right]-\mathbb{P}\left[X_{2} \leq F_{2}^{-1}(u)\right]+\mathbb{P}\left[X_{2} \leq F_{2}^{-1}(u), X_{1} \leq F_{1}^{-1}(u)\right]}{1-\mathbb{P}\left[X_{1} \leq F_{1}^{-1}(u)\right]}
$$

therefore we can present the following formula,

$$
\lambda_{\text {upper }}=\lim _{u \rightarrow 1} \frac{1-u-u+C(u, u)}{1-u} .
$$

Hence if $\left(X_{1}, X_{2}\right)^{\top}$ are upper tail independent then $\lambda_{\text {upper }}=0$, otherwise $\lambda_{\text {upper }} \in(0,1]$. Analogously, we can define the lower tail dependence as follows,

Definition 2.11 (Lower Tail Dependence). Let $\left(X_{1}, X_{2}\right)^{\top} \in \mathbb{R}^{2}$ be a continuous random vector and $X_{1} \sim F_{1}\left(x_{1}\right), X_{2} \sim F_{2}\left(x_{2}\right)$, then the coefficient of the upper tail dependence of $\left(X_{1}, X_{2}\right)^{\top}$ can be represented as follows,

$$
\lambda_{\text {lower }}=\lim _{u \rightarrow 0} \mathbb{P}\left[X_{2} \leq F_{2}^{-1}(u) \mid X_{1} \leq F_{1}^{-1}(u)\right]
$$

As $\mathbb{P}\left[X_{2} \leq F_{2}^{-1}(u) \mid X_{1} \leq F_{1}^{-1}(u)\right]$ in Formula (2.21) is equivalent to the formulation such that

$$
\frac{\mathbb{P}\left[X_{2} \leq F_{2}^{-1}(u), X_{1} \leq F_{1}^{-1}(u)\right]}{\mathbb{P}\left[X_{1} \leq F_{1}^{-1}(u)\right]},
$$

therefore we can give the following formula,

$$
\lambda_{\text {upper }}=\lim _{u \rightarrow 1} \frac{C(u, u)}{u} .
$$

\section{Monte Carlo Simulation in Copula}

For investigation of the dependence of a multivariate copula, the Monte Carlo simulation is helpful. Let $F: \mathbb{R}^{K} \rightarrow \mathbb{R}$ be the CDF of a random vector $\left(X_{1}, \ldots, X_{K}\right)^{\top} \in \mathbb{R}^{K}$, $F_{k}, k \in\{1, \ldots, K\}$ be the margins and $C$ be the corresponding $K$-dimensional copula, 
then the algorithm of the Monte Carlo simulation for the copula can be described as follows,

\section{Algorithm}

1. Generate a sample of $K$ uniform distributed random variables $\left(t_{m, 1}, \ldots, t_{m, K}\right)^{\top}, m \in$ $\{1, \ldots, M\}$ from $\left(T_{1}, \ldots, T_{K}\right)^{\top}$, where $T_{k} \sim$ Uniform $[0,1], k \in\{1, \ldots, n\}$.

2. Let $C_{k+1 \mid k}^{-1}(\cdot \mid \cdot), k \in\{1, \ldots, K\}$ be an inverse of a conditional copula function then let $u_{m, 1}=t_{m, 1}$ then we obtain

$$
u_{m, 2}=C_{2 \mid 1}^{-1}\left(t_{m, 2} \mid u_{m, 1}\right)
$$

Use the same routine we obtain

$$
u_{m, k}=C_{k \mid k-1, \ldots, 1}^{-1}\left(t_{m, k} \mid t_{m, k-1}, \ldots, t_{m, 1}\right), k \in\{2, \ldots, K\}
$$

Then we obtain a vector $\left(u_{m, 1}, \ldots, u_{m, K}\right)^{\top}$.

3. Then we have $\left(x_{m, 1}, \ldots, x_{m, K}\right)^{\top}=\left(F_{1}\left(u_{m, 1}\right)^{-1}, \ldots, F_{n}\left(u_{m, K}\right)^{-1}\right)^{\top}$.

4. Repeat $m \in\{1, \ldots, M\}$ to get $m$ rounds Monte Carlo simulations for the random vector $\left(X_{1}, \ldots, X_{K}\right)^{\top} \sim F$.

As an example of Monte Carlo simulation we show the random sampling by an exchangeable Gaussian copula with 6 different Pearson correlations as the parameters in Figure 2.1 .

\subsection{Elliptical Copula}

The elliptical family of copulas is widely used in finance studies. For instance in the study of CDO pricing papers by $\mathrm{Li}[1]$ and $\mathrm{Li}$ [2] a $K$-dimensional Gaussian copula is integrated in the modeling of dependence of default times, where the Gaussian copula is a member in the elliptical family of copulas, which bears the drawback of lacking the tail-dependence, which can be overcome by another member of the elliptical family copula, the Student- $t$ copula. In this section we give a brief view of the elliptical family of copulas.

Definition 2.12 (Elliptical Distribution). A $K$-dimensional random vector $X=\left(X_{1}\right.$, $\left.\ldots, X_{K}\right)^{\top}$ is said elliptically distributed such that $X \sim \varepsilon_{K}(\Delta, \Sigma)$, where $\Delta \in \mathbb{R}^{K}$ and 


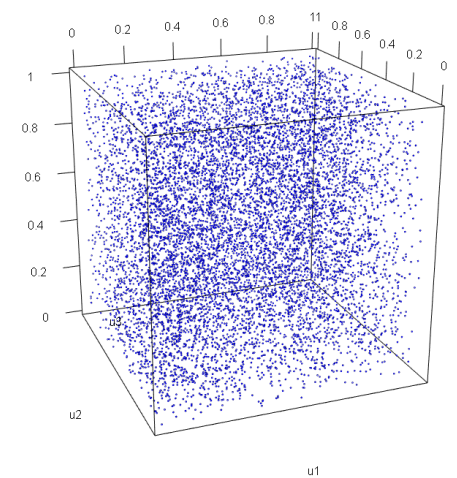

(a) $\rho=0.1$

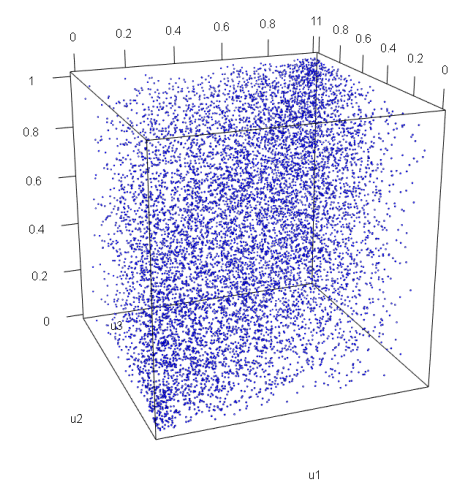

(c) $\rho=0.4$

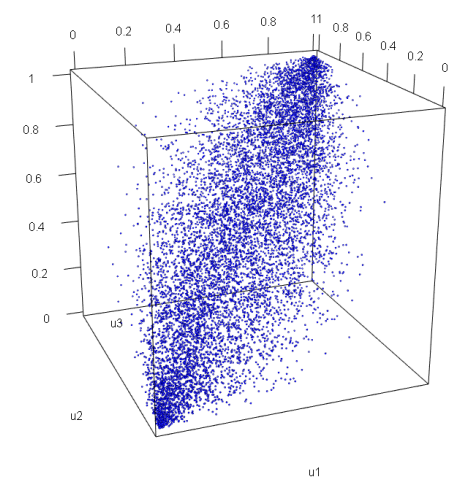

(e) $\rho=0.8$

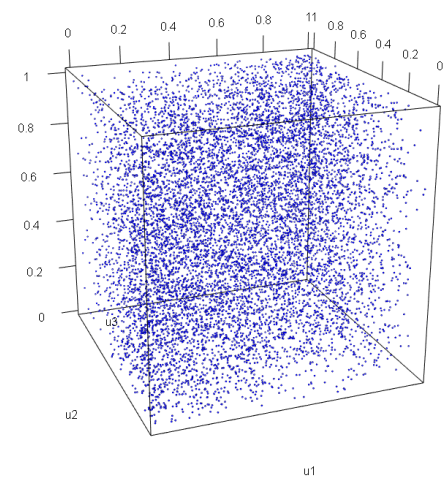

(b) $\rho=0.2$

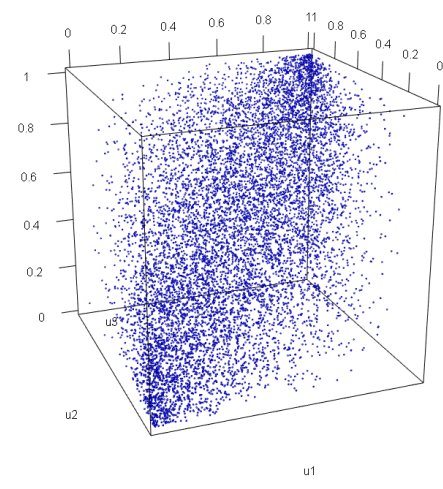

(d) $\rho=0.6$

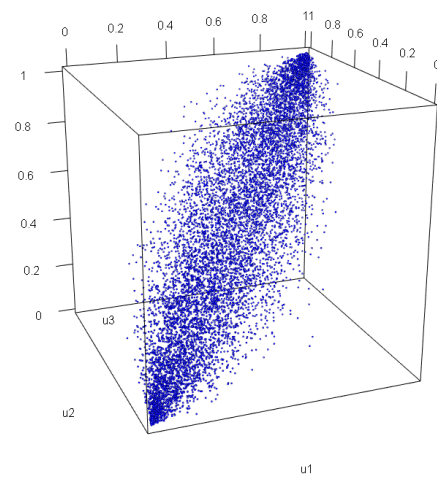

(f) $\rho=0.9$

Figure 2.1: 10000 Monte Carlo simulations for 3-dimensional Gaussian copula with an exchangeable correlation matrix.

Qmargss.R 
$\Sigma$ is a $(K \times K)$ positive definite symmetric matrix, if the PDF of $X$ can be represented as the following form such that

$$
f(x)=D_{K}|\Sigma|^{-\frac{1}{2}} \Lambda\left((x-\Delta)^{T} \Sigma^{-1}(x-\Delta)\right)
$$

where $x=\left(x_{1}, \ldots, x_{K}\right)^{\top}, \Lambda: \mathbb{R} \rightarrow \mathbb{R}$ and $D_{K}$ is a constant factor.

\section{Gaussian Copula}

The Gaussian copula can be constructed by an employment of the Sklar's Theorem 2.3 by embedding all inverses of the marginal CDFs in a $K$-dimensional CDF such as the following definition.

Definition 2.13 (Gaussian Copula). For a $K$-dimensional uniform vector $u=\left(u_{1}, \ldots\right.$, $\left.u_{K}\right)^{T} \in I^{K}=[0,1]^{K}$, the Gaussian copula can be represented as follows,

$$
C_{g s}(u ; \rho)=\Phi_{K}\left(\Phi^{-1}\left(u_{1}\right), \ldots, \Phi^{-1}\left(u_{K}\right) ; \rho\right)
$$

where $\rho$ is a $(K \times K)$ correlation matrix, $\Phi_{K}()$ is a $K$-dimensional standard normal distribution function and $\Phi()$ is a 1-dimensional standard normal distribution function.

For the simulation of the Gaussian copula under the 2 dimension case we refer to Figure $2.2(a)$ and for its density and density contour we refer to Figure $2.2(g)$ and $(d)$.

\section{Student- $t$ Copula}

Analogous to the Gaussian copula, the Student- $t$ copula can also be constructed by an employment of the Sklar's Theorem 2.3 by embedding all inverses of the marginal CDFs in an $K$-dimensional CDF.

Definition 2.14 (Student- $t$ Copula). Let $\nu \in(1,+\infty)$ the degree of freedom and $\rho=$ $\left(1-\frac{2}{\nu}\right) \operatorname{var}(X)$ the $(K \times K)$ dispersion matrix, $X=\left(X_{1}, \ldots, X_{K}\right)^{\top} \in \mathbb{R}^{K}$, then the $K$-dimensional Student- $t$ distribution $t_{K}(x)$ can be represented such that

$$
t_{K}(x)=\frac{\Gamma\left(\frac{\nu+K}{2}\right)}{\frac{\nu}{2} \sqrt{(\Pi \nu)^{K}|\rho|}}\left(1+\frac{(x-\Delta)^{T} \rho^{-1}(x-\Delta)}{\nu}\right)^{-\frac{\nu+K}{2}}
$$


where $\Gamma(y)=\int_{0}^{+\infty} x^{y} e^{-x} \frac{\mathrm{d} x}{x}, \Delta=\mathbb{E}(X)$ and $x=\left(x_{1}, \ldots, x_{K}\right)$, then the Student- $t$ Copula can be represented as follows,

$$
C_{t}(u ; \nu, \Delta, \rho)=t_{K}\left(t^{-1}\left(u_{1} ; \nu\right), \ldots, t^{-1}\left(u_{K} ; \nu\right) ; \nu, \Delta, \rho\right)
$$

where $t_{K}()$ is an $K$-dimensional Student- $t$ distribution function and $t^{-1}()$ is an inverse of a 1-dimensional Student- $t$ distribution function.

For the simulation of the Student- $t$ copula under the 2 dimension case we refer to Figure $2.2(b)$ and for its density and density contour we refer to Figure $2.2(h)$ and $(e)$.

\subsection{Archimedean Copula}

A frequently studied topic in finance is the crisis contagion which investigates the joint behavior between different markets, for instance in $\mathrm{Hu}$ [34], which shows that the dependence between four market indices including SP 500, FTSE, Nikkei and Hang Seng is not symmetric and can be interpreted as a left tail dependence which is the very feature of the Clayton copula belonging to a member in the Archimedean family of copulas. Next we introduce the Archimedean family of copulas and its members.

Definition 2.15 (Archimedean Copula). Let $\varphi:[0,1] \rightarrow[0,+\infty)$ is the generator function, which satisfies the following three properties,

1. $\varphi(1)=0$,

2. $\varphi(+\infty)=1$,

3. $\varphi:[0,1] \rightarrow[0,+\infty)$ is a decreasing function.

Then the representation of the Archimedean copula can be given as follows,

$$
C\left(u_{1}, \ldots, u_{n} ; \theta\right)= \begin{cases}\varphi^{-1}\left(\varphi\left(u_{1} ; \theta\right)+\cdots+\varphi\left(u_{K} ; \theta\right) ; \theta\right) & \text { if } \sum_{k=1}^{K} \varphi\left(u_{k} ; \theta\right) \leq \varphi(0 ; \theta) \\ 0 & \text { else. }\end{cases}
$$

For illustration of the members of Archimedean copula family we show four 2-dimensional examples of the Frank copula, the Clayton copula, the Gumbel copula and the Joe copula. Their random samples, density contours and densities are respective given in Figure 2.2 and Figure 2.3. 


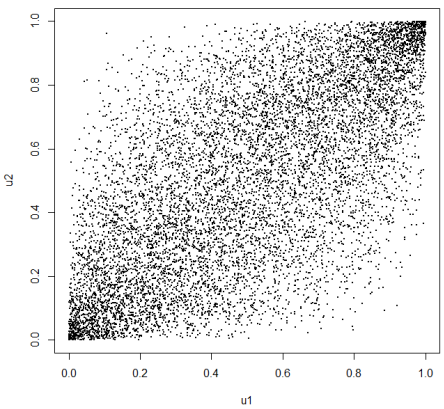

(a)

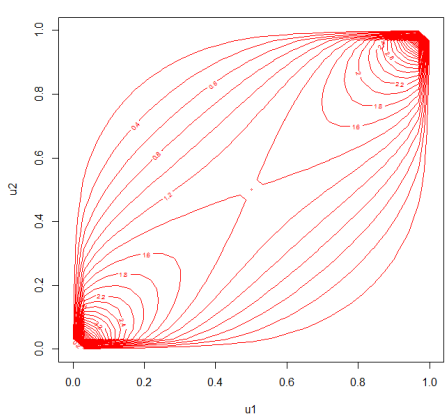

(d)

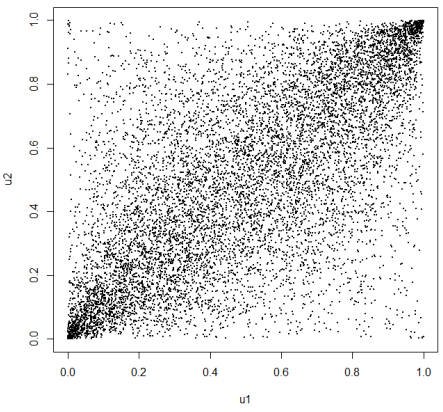

(b)

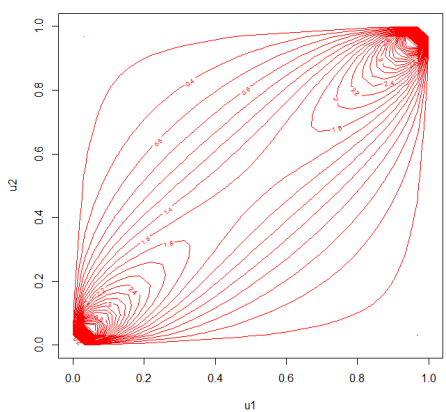

(e)

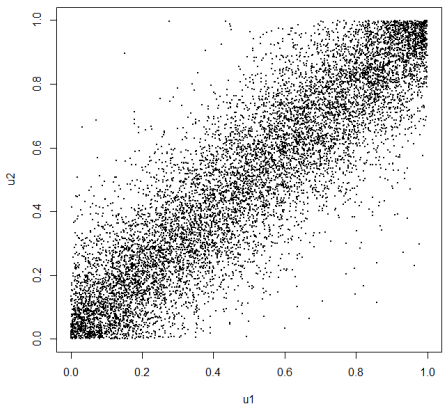

(c)

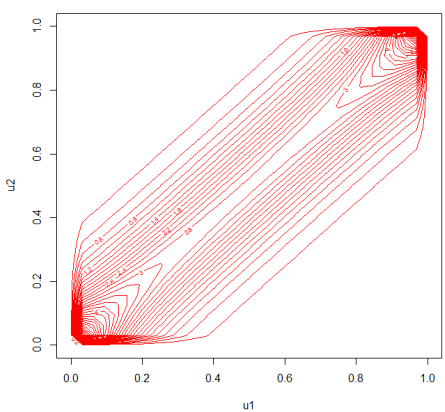

(f)

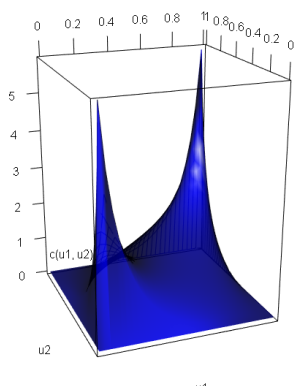

(g)

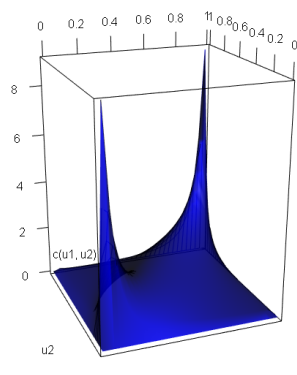

(h)

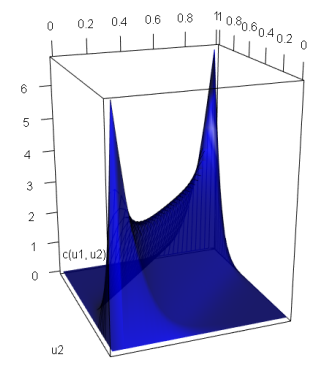

(i)

FiguRE 2.2: The first row is for 2-dimensional scatter plots, whose points are drawn from copulas. The second row is for 2-dimensional contour plots of copula densities. The third row is for 2-dimensional copula density plots. From left to right, the first column is for the Gaussian copula with $\rho_{\mathcal{P}}=0.7$. The second column is for the Student$t$ copula with $\rho_{\mathcal{P}}=0.7$ and the degree of freedom equal to 3 . And the third column is for the Frank copula with $\rho_{\mathcal{K}}=0.7$.

QMARpdfgstfrank.R 


\section{Ali-Mikhail-Haq Copula}

Definition 2.16 (Ali-Mikhail-Haq Copula). Let the generator function $\varphi$ such that

$$
\varphi(y ; \theta)=\ln \left(\frac{1-\theta(1-y)}{y}\right), \theta \in[-1,1]
$$

then by using the Formula (2.30) the Ali-Mikhail-Haq copula can be represented as follows,

$$
C_{a}\left(u_{1}, \ldots, u_{K} ; \theta\right)=\frac{\prod_{k=1}^{K} u_{k}}{1-\theta\left(\prod_{k=1}^{K}\left(1-u_{k}\right)\right)}
$$

\section{Frank Copula}

Definition 2.17 (Frank Copula). Let the generator function $\varphi$ such that

$$
\varphi(y ; \theta)=-\log \left(\frac{\exp (-\theta y)-1}{\exp (-\theta)-1}\right), \theta \in(0,+\infty)
$$

then by using the Formula (2.30) the Frank copula can be represented as follows,

$$
C_{f}\left(u_{1}, \ldots, u_{K} ; \theta\right)=-\frac{1}{\theta} \log \left[1+\frac{\prod_{k=1}^{K}\left(\exp \left(-\theta u_{k}\right)-1\right)}{(\exp (-\theta)-1)^{K-1}}\right]
$$

\section{Clayton Copula}

Definition 2.18 (Clayton Copula). Let the generator function $\varphi$ such that

$$
\varphi(y ; \theta)=\frac{1}{\theta}\left(y^{-\theta}-1\right), \theta \in[-1, \infty) \backslash\{0\},
$$

then by using the Formula (2.30) the Clayton copula can be represented as follows,

$$
C_{c}\left(u_{1}, \ldots, u_{K} ; \theta\right)=\left[\left(\sum_{k=1}^{K} u_{i}^{-\theta}-K+1\right)\right]^{-\frac{1}{\theta}} .
$$




\begin{tabular}{l|l|l|l}
\hline \hline Member & Copula & Generator & Parameter \\
\hline Ali-Mikhail-Haq & $\frac{\prod_{k=1}^{K} u_{k}}{1-\theta\left(\prod_{k=1}^{K}\left(1-u_{k}\right)\right)}$ & $\ln \left(\frac{1-\theta(1-y)}{y}\right)$ & $\theta \in[-1,1]$ \\
Frank & $-\frac{1}{\theta} \log \left[1+\frac{\prod_{k=1}^{K}\left(\exp \left(-\theta u_{k}\right)-1\right)}{(\exp (-\theta)-1)^{K-1}}\right]$ & $-\log \left(\frac{\exp (-\theta y)-1}{\exp (-\theta)-1}\right)$ & $\theta \in(0,+\infty)$ \\
Clayton & {$\left[\left(\sum_{k=1}^{K} u_{k}^{-\theta}-K+1\right)\right]^{-\frac{1}{\theta}}$} & $\frac{1}{\theta}\left(y^{-\theta}-1\right)$ & $\theta \in[-1, \infty) \backslash\{0\}$ \\
Gumbel & $\exp \left[-\sum_{k=1}^{K}\left(-\ln u_{k}\right)^{\theta}\right]^{\frac{1}{\theta}}$ & $(-\ln (y))^{\theta}$ & $\theta \in[1,+\infty)$ \\
Joe & $1-\left[\sum_{k=1}^{K}\left(1-u_{k}\right)^{\theta}-\prod_{k=1}^{K}\left(1-u_{k}\right)^{\theta}\right]^{\frac{1}{\theta}}$ & $-\log \left(1-(1-y)^{\theta}\right)$ & $\theta \in[1,+\infty)$ \\
\hline \hline
\end{tabular}

TABle 2.1: Five Archimedean copula functions including the Ali-Mikhail-Haq copula, the Frank copula, the Clayton copula, the Gumbel copula and the Joe copula, generator functions and parameters.

\section{Gumbel Copula}

Definition 2.19 (Gumbel Copula). Let the generator function $\varphi$ such that

$$
\varphi(y ; \theta)=(-\ln (y))^{\theta}, \theta \in[1,+\infty)
$$

then by using the Formula (2.30) the Gumbel copula can be represented as follows,

$$
C_{g}\left(u_{1}, \ldots, u_{K} ; \theta\right)=\exp \left[-\sum_{k=1}^{K}\left(-\ln u_{i}\right)^{\theta}\right]^{\frac{1}{\theta}}
$$

\section{Joe Copula}

Definition 2.20 (Joe Copula). Let the generator function $\varphi$ such that

$$
\varphi(y ; \theta)=-\log \left(1-(1-y)^{\theta}\right), \theta \in[1,+\infty)
$$

then by using the Formula (2.30) the Joe copula can be represented as follows,

$$
C_{j}\left(u_{1}, \ldots, u_{K} ; \theta\right)=1-\left[\sum_{k=1}^{K}\left(1-u_{k}\right)^{\theta}-\prod_{k=1}^{K}\left(1-u_{k}\right)^{\theta}\right]^{\frac{1}{\theta}}
$$

We conclude this section with the following Table 2.1. 


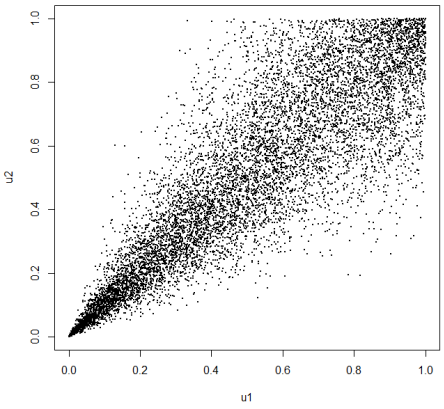

(a)

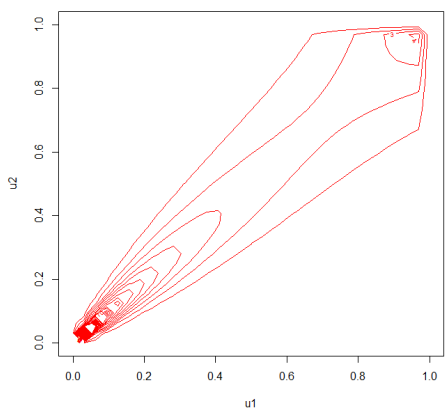

(d)

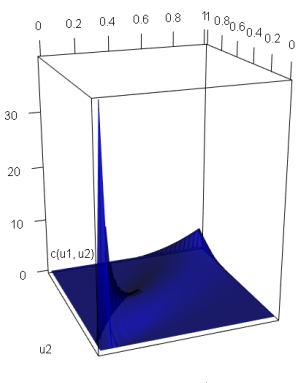

(g)

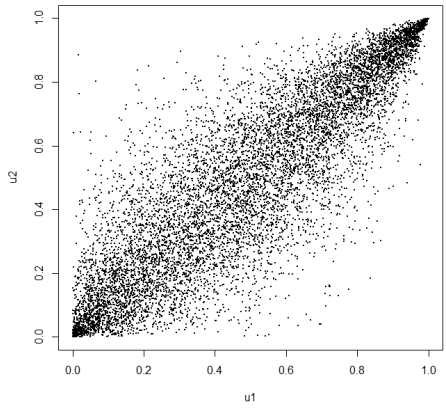

(b)

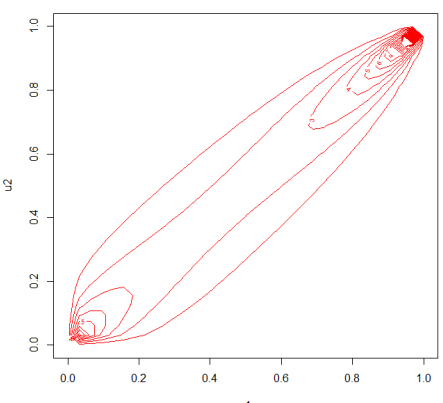

(e)

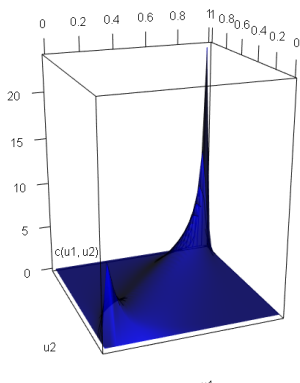

(h)

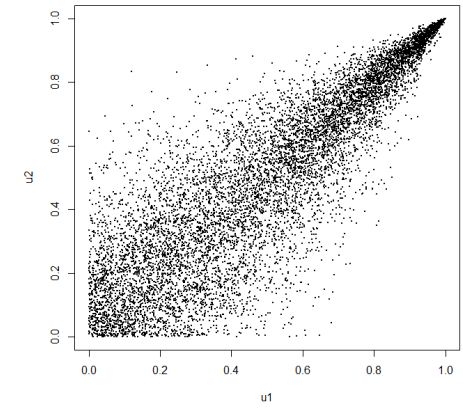

(c)

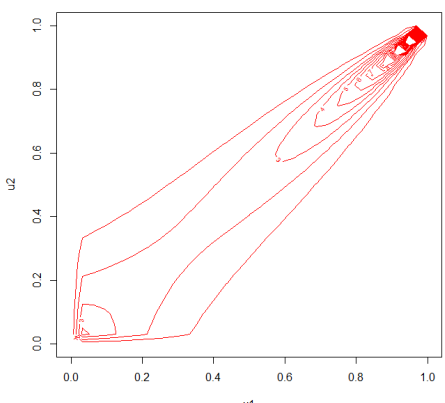

(f)

(i)

FIGURE 2.3: The first row is for 2-dimensional scatter plots, whose points are drawn from copulas. The second row is for 2-dimensional contour plots of copula densities. The third row is for 2-dimensional copula density plots. From left to right, the first column is for the Clayton copula with $\rho_{\mathcal{P}}=0.7$. The second column is for the Gumbel copula with $\rho_{\mathcal{P}}=0.7$. And the third column is for the Joe copula with $\rho_{\mathcal{K}}=0.7$.

OMARpdfclaytongumbeljoe.R 


\subsection{Hierarchical Archimedean Copula}

The above introduced Archimedean copulas (AC) have pros such that the dependence structure can be non-elliptical, for instance the Gumbel copula, the Clayton copula and the Joe copula, and also tail-dependent, which are fit for modeling in finance, for instance in the modeling of equity returns, however ACs also show contras in heterogeneity of dependence which is lead by the exchangeability that there exists only one parameter in exchangeable ACs to control the joint behavior, which is not reasonable in the practice. Hence we introduce here another copula family, the hierarchical Archimedean family (HAC) which is also termed as the nested Archimedean copula (NAC) in Hofert and Scherer [8] and Hofert [9]. For details of the hierarchical Archimedean copula we refer to Okhrin et al. [35].

Definition 2.21 (Hierarchical Archimedean Copula). A $K$-dimensional copula $C$ is defined as the hierarchical Archimedean copula if itself is an Archimedean copula and its arguments can be replaced by other hierarchical Archimedean copulas.

Definition 2.22 (Fully Hierarchical Archimedean Copula).

$$
\begin{aligned}
C\left(u_{1}, \ldots, u_{K}\right)= & \left.\left.C\left[C\left(\ldots C\left(u_{1}, u_{2} ; \varphi_{1}\right), u_{3} ; \varphi_{2}\right), \cdots, u_{K-1} ; \varphi_{K-2}\right)\right), u_{K} ; \varphi_{K-1}\right] \\
= & \varphi_{K-1}\left[\varphi _ { K - 1 } ^ { - 1 } \left(\varphi _ { K - 2 } \left(\ldots\left(\varphi_{2}^{-1}\left(\varphi_{1}\left(\varphi_{1}^{-1}\left(u_{1}\right)+\varphi_{1}^{-1}\left(u_{2}\right)\right)\right)+\varphi_{2}^{-1}\left(u_{3}\right)\right)\right.\right.\right. \\
& \left.\left.\left.+\cdots+\varphi_{K-2}^{-1}\left(u_{K-1}\right)\right)\right)+\varphi_{K-1}^{-1}\left(u_{K}\right)\right] .
\end{aligned}
$$

Definition 2.23 (Partially Hierarchical Archimedean Copula).

$$
\begin{aligned}
C\left(u_{1}, \ldots, u_{K}\right)= & C\left(C\left(u_{11}, \ldots, u_{1 K_{1}} ; \varphi_{1}\right), \ldots, C\left(u_{I 1}, \ldots, u_{I K_{I}} ; \varphi_{I}\right) ; \varphi\right) \\
= & \varphi\left(\varphi^{-1}\left[\varphi_{1}\left(\varphi_{1}^{-1}\left(u_{11}\right)+\cdots+\varphi_{1}^{-1}\left(u_{1 K_{1}}\right)\right)\right]+\cdots\right. \\
& \left.+\varphi^{-1}\left[\varphi_{I}\left(\varphi_{I}^{-1}\left(u_{I 1}\right)+\cdots+\varphi_{I}^{-1}\left(u_{I K_{I}}\right)\right)\right]\right) \\
= & \varphi\left(\sum_{k=1}^{I} \varphi^{-1}\left\{\varphi_{k}\left[\sum_{j=1}^{K_{k}} \varphi_{k}^{-1}\left(u_{k j}\right)\right]\right\}\right),
\end{aligned}
$$

where $u_{k j} \in[0,1], k \in\{1, \ldots, I\}, j \in\left\{1, \ldots, K_{k}\right\}$.

The structure of an HAC can be illustrated with a tree graph. For the above referred fully $\mathrm{HAC}$ and partially $\mathrm{HAC}$ we present a tree graph example under a five-dimensional context showing in Figure $2.4(a)$. In Figure $2.4(b)$ the highest Archimedean copula 
$\left(C_{(12)(345)}\right)$ is often termed as the root copula, and if an Archimedean copula in the tree structure has at least one hierarchical Archimedean copula then it is called the parent copula and the corresponding hierarchical copulas are referred as the child copulas. Therefore in the Figure 2.4 lower graph the $C_{(12)(345)}$ represents the parent copula and the $C_{12}$ and the $C_{345}$ represent the child copulas.

The HAC plays an important role in finance as it can be used to construct the dependence structures taking an account on the groups, taking the lower graph in Figure 2.4 as an example that we assume that $\left(U_{1}, U_{2}\right)^{\top}$ have left tail-dependence and $\left(U_{3}, U_{4}, U_{5}\right)^{\top}$ have right tail-dependence then the $\left(U_{1}, U_{2}\right)^{\top}$ can be modeled by a Clayton copula (c.f. the Formula $(2.36)$ ) and the $\left(U_{3}, U_{4}, U_{5}\right)^{\top}$ can be modeled by a Gumbel copula (c.f. the Formula (2.38)) or a Joe copula (c.f. the Formula (2.40)) and at last the two new copulas can be coupled with $C\left(C\left(u_{1}, u_{2} ; \varphi_{1}\right), C\left(u_{3}, u_{4}, u_{5} ; \varphi_{2}\right) ; \varphi_{3}\right)$, hence the heterogeneity of dependence between different groups is already considered, which can not be accomplished in the context of the exchangeable Archimedean copulas or the elliptical copulas.

\subsection{Mixed Copula}

Next we consider another copula family, the mixed copula, which is a linear combination of copulas. The definition is given as follows.

Definition 2.24 (Mixed Copula). A mixed copula is a linear combination of copulas, which can be defined with the following formula,

$$
C_{m i x}(u ; \theta)=\sum_{d=1}^{f} \lambda_{d} C_{d}\left(u ; \theta_{d}\right)
$$

where $u=\left(u_{1}, \ldots, u_{K}\right), \theta$ the parameter set of the mixed copula $C, \lambda_{d}$ the $d$-th weight and $\sum_{d=1}^{f} \lambda_{d}=1, C_{d}\left(u ; \theta_{d}\right)$ the $d$-th component copula with a parameter set $\theta_{d}, d \in$ $\{1, \ldots, f\}$ the index of the component copula and $f$ the number of component copulas.

It is not difficult to show that the formula defined in (2.43) is also a copula under the copula definition in 2.1. Therefore $C(u ; \theta)$ can be seen as an unknown copula (or a complex copula) composed by known copulas $C_{d}\left(u ; \theta_{d}\right), d \in\{1, \ldots, f\}$, hence the mixed copula $C(u ; \theta)$ should inherit features from its component copulas, which is practical and 


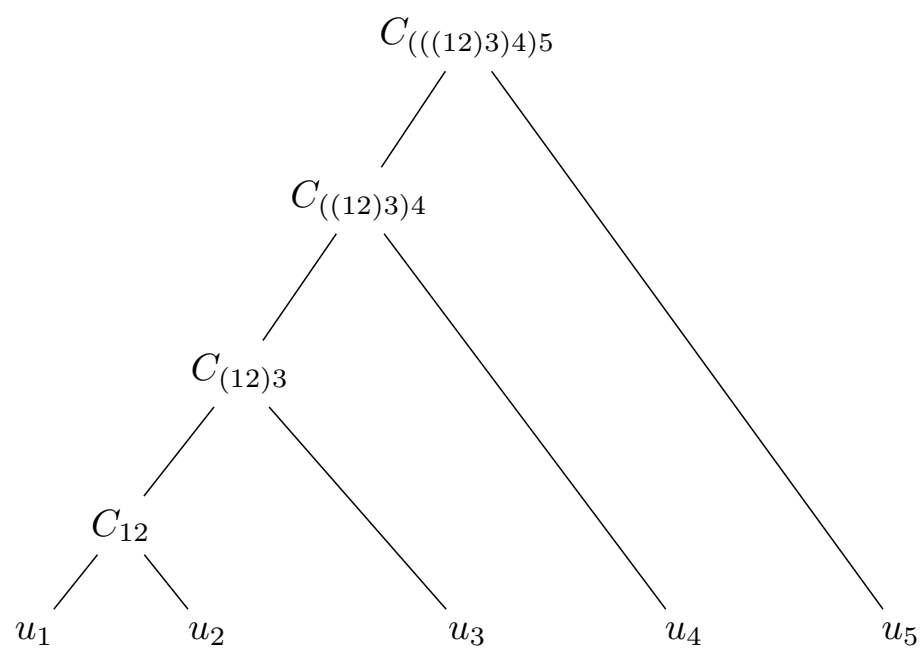

$C\left(u_{1}, u_{2}, u_{3}, u_{4}, u_{5}\right)=C\left(C\left(C\left(C\left(u_{1}, u_{2} ; \varphi_{1}\right), u_{3} ; \varphi_{2}\right), u_{4} ; \varphi_{3}\right), u_{5} ; \varphi_{4}\right)$

(a)

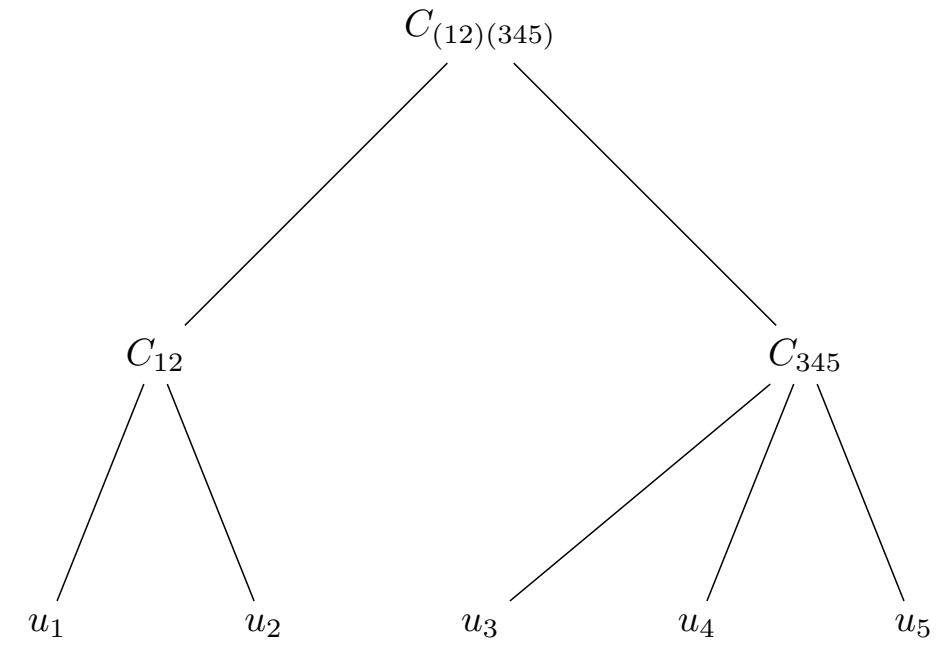

$C\left(u_{1}, u_{2}, u_{3}, u_{4}, u_{5}\right)=C\left(C\left(u_{1}, u_{2} ; \varphi_{1}\right), C\left(u_{3}, u_{4}, u_{5} ; \varphi_{2}\right) ; \varphi_{3}\right)$

(b)

FiguRE 2.4: Tree structures of two hierarchical Archimedean copulas, where the upper tree shows the fully hierarchical Archimedean copula and the lower tree shows the partially hierarchical Archimedean copula under 5-dimensional copula context. 
reasonable in finance for capturing different joint behaviors such as the heterogeneity of dependence and the asymmetrical tail-dependence.

In the aspect of application of the mixed copula, $\mathrm{Hu}$ [34] has studied the mixed copulas and applied a bivariate Gaussian-Gumbel-survival mixed copula in modeling the dependence of stock markets. In this paper four stock market indices including SP, FTSE, Nikkei and Hang Seng have been investigated with the bivariate mixed copula, the Gaussian-Gumbel-survival mixed copula, which shares the left-tail dependence from the Gumble survival copula, and arrived a conclusion that the pairwise markets have high probability to crash together not to boom together which coincides with the left tail-dependence. Another two papers, Wang [36] and Cai et al. [37] also focus on the mixed copulas. Especially for Cai et al. [37], the author has contributed a copula tuning method for copula selection which is based on the penalized likelihood that the parameters in the mixed copula and component choice can be accomplished together similar to LASSO. In empirical part the Monte Carlo simulations and the construction of the financial time series are performed.

Later in this master thesis we employ the mixed copulas with 2 component copulas, which are used to specify the dependence structures of default times of entities in the underlying pool for utilizing the features from the component copulas in the mixed copula model. 


\section{Chapter 3}

\section{CDO}

In the study of the credit derivatives, CDS and CDO are two core instruments, whose main purposes are transferring credit risk between counterparts, i.e. creditors and credit investors. Both instruments can be seen as the credits protection technology, however the both are different in their mechanisms. Simply to say, the CDS is used as an instrument for providing protection for a single name credit while the CDO is designed as a protection technique for a credit portfolio.

In this chapter we introduce firstly the CDS and its related products. In the second section we give an introduction of the CDO, especially the synthetic CDO. Then in the third section we focus on the CDS index tranche.

\subsection{CDS and Basket Default Swap}

\section{Credit Default Swap (CDS)}

In the structure of a CDS contract or a single name CDS contract there exist two counterparts, i.e. a protection seller and a protection buyer. Normally when the a creditor bears credit risk exposure, then in order to hedge this risk the creditor enters into a CDS contract as a protection buyer and need to pay swap premiums according to the regularized dates underwritten in the contract. And correspondingly a protection seller also enters into this CDS contract to provide the protection buyer the default protection that when a credit event of the underlying written in the contract happens 
then the protection seller will deliver the compensation to the protection buyer for making up the loss in the credit event. In a CDS contract credit events and swap

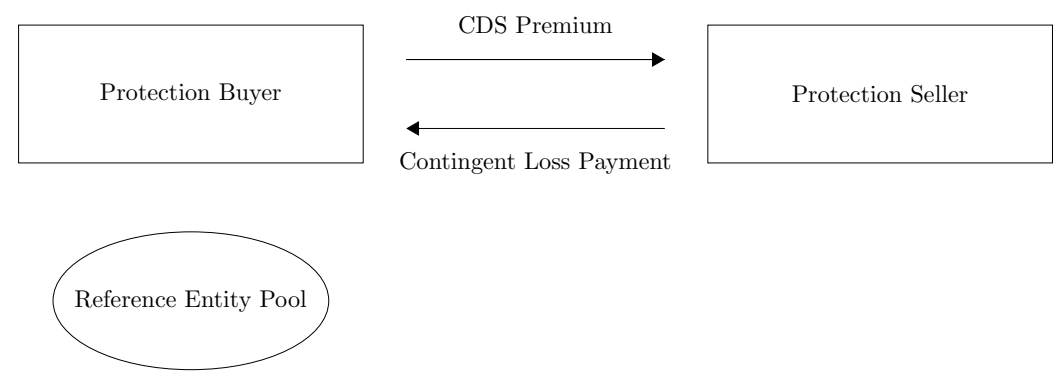

FIGURE 3.1: The structure of a credit default swap (CDS), which provides the creditor a single name protection from default.

premiums are pivotal clauses. Some definitions will be given in a CDS contract for credit events, for instance that based on publicly reported financial indicators a corporate can be defined as the bankruptcy. All these credit events are triggers of contingent premium payments and compensation deliveries. The swap premiums are payed serially according to the payment dates written in the contract, for example quarterly payment, half yearly payment or yearly payment.

When a CDS contract runs then two cases will be considered, i.e. credit events occur or not occur. In the first case the referred entity in the contract survives the maturity then the protection buyer will pay all the protection fee, i.e. the swap premiums according to the payment dates and as no loss happens hence the protection seller will pay no compensation to its counterpart. In the second case the clauses in the contract trigger credit events, then the protection buyer will obtain the compensation from the protection seller for the loss by the referred entity with one of two different methods, the physical settlement, in which the protection buyer delivers the physical entity, for instance credit bonds or loans to the protection seller and obtains from the protection seller the notional principal invested in this referred entity, and the cash settlement, in which the protection buyer obtains the difference between the principal and the market value of the underlying from the protection seller.

\section{Basket Default Swap}

The single name CDS is an innovation for the credit risk hedging, however it has a drawback for that it is suitable only for a single entity not a portfolio. In the context 
of a multi-name credit portfolio people can take account on the basket default swap which can provide multi-name protection. In such product family the $k$ th-to-default swap, $k \in\{1, \ldots, K\}$, are frequently utilized, in which the protection seller will pay its counterpart the compensation of loss by the reference entity only when the $k$ th entity of the underlying pool triggers the credit event. After settlement between the counterparts the contract will be terminated. The main difference between the single-name CDS and the $k$ th-to-default swap is the triggering condition, where the former focuses only on a single underlying while the later on the $k$ th in a credit basket.

\subsection{Synthetic CDO and Cash CDO}

As the above we have already introduced a product for credit portfolio protection, the $k$ th-to-default swap, which can be seen as a product providing an interval protection in $[0, k / K], k \in\{1, \ldots, K\}$, therefore only one attachment point 0 and one detachment point $k / K$ are considered. Here we introduce another product for providing protection for credit portfolio, the collateralized debt obligation (CDO), which can provide protection buyers several attachment points and detachment points by the slicing technology which divides a whole credit portfolio into several tranches, each of which provides a protection range defined by an interval. In the market there are two CDO categories which are widely used, which are called the cash CDO and the synthetic CDO.

The structure of a collateralized debt obligation (CDO) is similar to other asset-backed securities (ABS), however the significant difference between a CDO and other ABS' is that the pool of underlying entities in a CDO can be input with physical debt obligations such as bonds and loans, which are correspondingly termed as the collateralized bond obligation (CBO) and the collateralized loan obligation (CLO), and also the structured financial instruments such as ABS, CDS and CDO, which are individually termed as the $\mathrm{CDO}$ of $\mathrm{ABS}, \mathrm{SCO}$ and $\mathrm{CDO}^{2}$. Usually CDOs are initiated by an originator, which can be financial institutions such as banks and asset management companies. As the reason of avoiding the association of credit risk from the sponsor, a new entity called the special purpose vehicle (SPV) will be constructed for the operation of the CDOs. If the SPV purchases the credit assets from the originator then the issued CDO will be termed as the cash CDO else if the SPV does not own physical debt obligations as assets but owns CDS contracts then the CDO will be named as the synthetic CDO. 


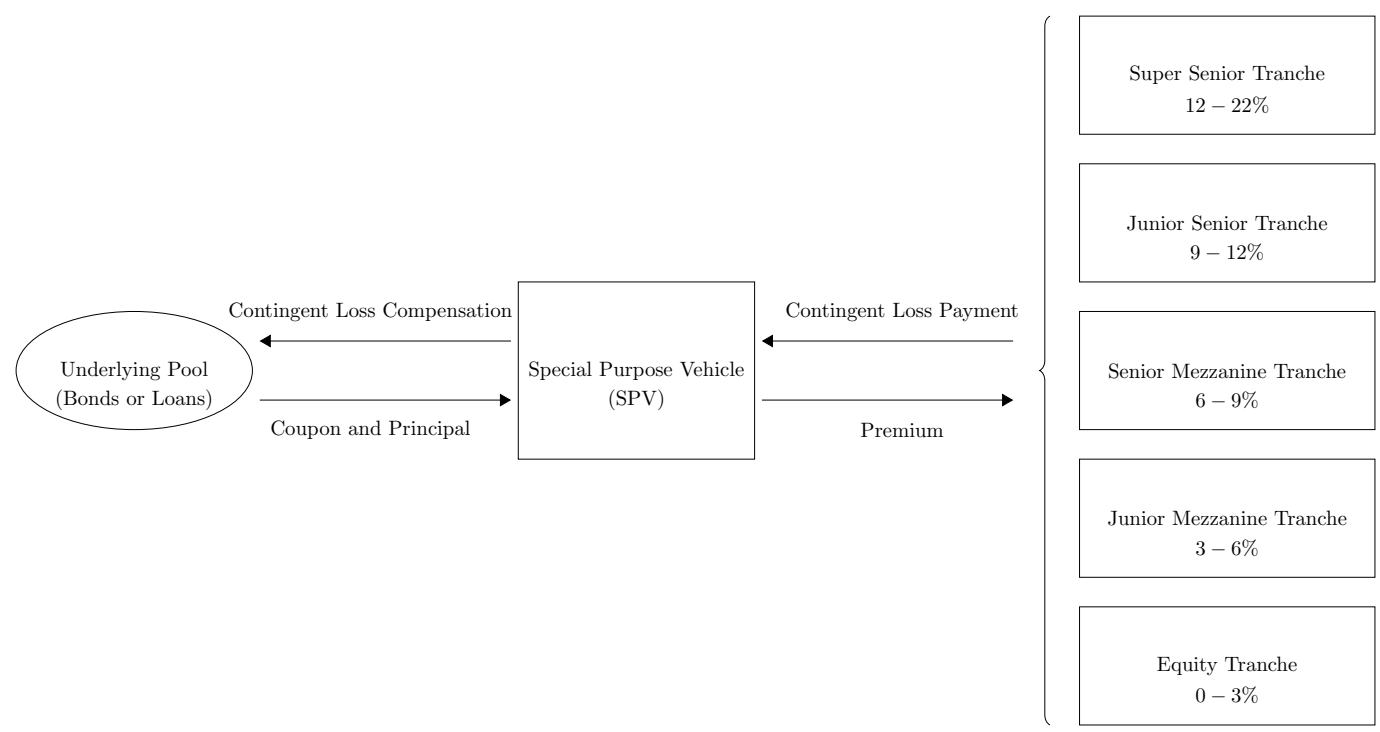

Figure 3.2: The structure of a cash collateralized debt obligation, whose main characteristic is the ownership of the reference assets in the underlying pool.

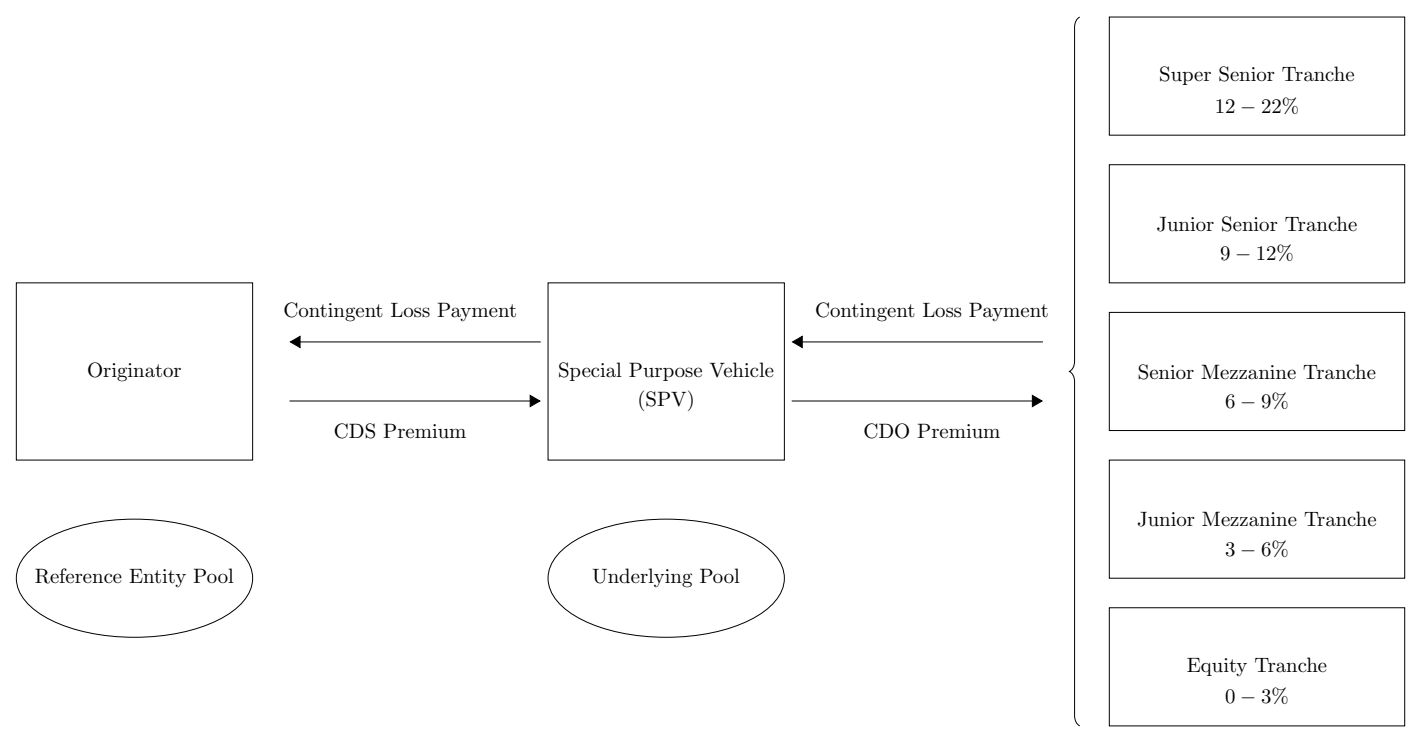

FIGURE 3.3: The structure of a synthetic collateralized debt obligation, whose main characteristic is the selling of CDS contracts and no ownership of the reference assets. 


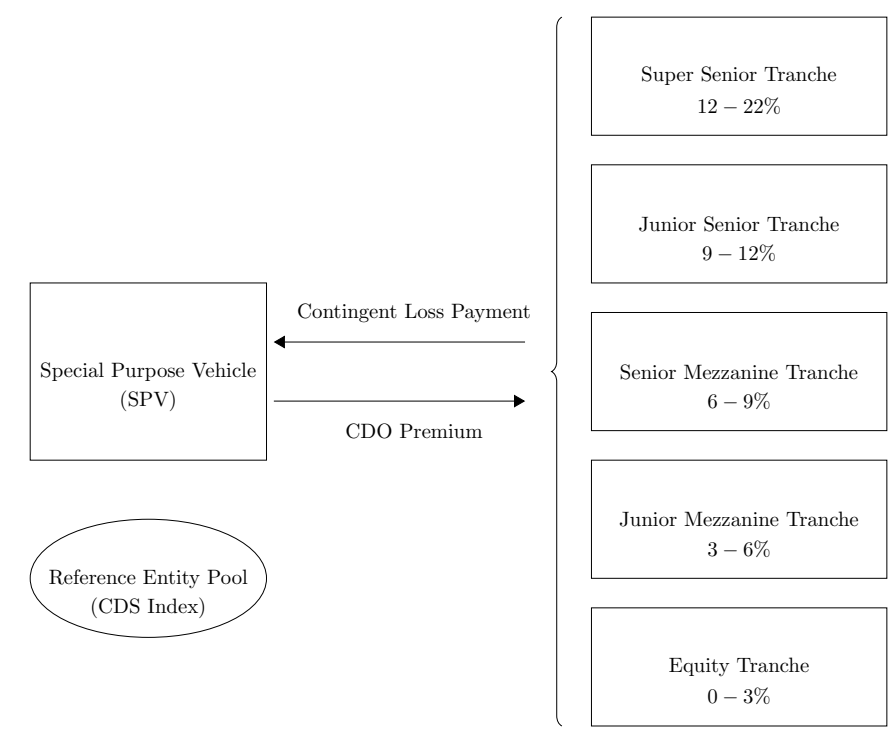

FigurE 3.4: The structure of CDS index tranches, whose main feature is selling the insurance-like policy for protection of the basket references.

In Figure 3.2 and Figure 3.3 we have shown the structure of a cash CDO and a synthetic CDO correspondingly. In Figure 3.2 the right side we see five tranches under an order from the lowest seniority, the Equity Tranche, to the highest, the Super Senior Tranche. Different seniority stands for different risk quantity that the higher the seniority the lower the risk and the risk premium. For example, an investor has bought the second tranche with $(3 \%, 6 \%]$, if there is no credit event occurs in the CDO running period then the investor as a protection seller will obtain all premiums from the protection buyer but if the aggregated loss is $4 \%$ higher than $3 \%$ and lower than $6 \%$ then the investor starts losing money and can only obtain the premium recalculated according to $6 \%-4 \%=2 \%$. But for the holder of tranche with $(12 \%, 22 \%]$ there is no influence as the lower senior tranches' has already absorbed the losses.

\subsection{CDS Index Tranche}

The credit default swap index tranche (CDS index tranche) is similar to the synthetic CDO which provides the protection buyer and seller an insurance policy-like contract. The difference between the synthetic CDO and the CDS index tranche is that the former obtains the credit risk by selling CDS contracts but the CDS index tranche does not sell CDS, which can be seen as a pure insurance policy betting on the occurrence of credit 


\begin{tabular}{c|lllll}
\hline \hline Interval & $0-3 \%$ & $3-6 \%$ & $6-9 \%$ & $9-12 \%$ & $12-22 \%$ \\
\hline spreads 5y & 16.67 & 106.42 & 45.945 & 28 & 17.5 \\
spreads 7y & 26.43 & 166.715 & 80.16 & 45.9 & 29.575 \\
\hline \hline
\end{tabular}

TABLE 3.1: Spreads of tranches of the iTraxx Europe Series 8 on 2007-10-23 with maturities of 5 years and 6 years sourced from the Bloomberg Terminal.

events of the reference entities. However as the cash flow structure of the CDS index tranche is same as the synthetic CDO hence both can be priced with the same routine.

In the market there are several CDS index tranche products, of which two products, the Dow Jones CDX NA IG and the iTraxx Europe, are traded frequently. Both products have five layers which are separated by attachment points and detachment points. We take the the iTraxx Europe Series 8 as an example. In the Table a five year and a seven year CDS index tranches are given. Here we need to note that in the iTraxx Europe there are five tranches traded in the market including the equity tranche $[0,3 \%]$, the junior mezzanine tranche $(3 \%, 6 \%]$, the senior mezzanine tranche $(6 \%, 9 \%]$, the junior senior tranche $(9 \%, 12 \%]$ and the super senior tranche $(12 \%, 22 \%]$. Especially for the equity tranche in Table 3.1, taking the 5 year as an example, the value 16.67 is a percentage which stands for the upfront payment rate and the value 100.42 for the the junior mezzanine tranche $(3 \%, 6 \%$ ] stands for $100.42 / 10000$ which is the premium payment rate for every period. And for the equity tranche the protection buyer will pay not only the upfront payment but also 500 basis points $(=500 / 10000)$ for every year and the other senior tranche pays only premium quarterly. 


\section{Chapter 4}

\section{CDO Pricing}

\subsection{Mechanism of CDO Pricing}

Firstly, we let a credit portfolio contain $K$ reference entities with overall $N$ notional principal and the notional be equally distributed on entities, i.e. every entity shares $1 / K$ of the overall investment. Meanwhile we let the maturity of the CDS index tranches be $T$, the length of the contract, and premiums will be payed at points $t_{j}, j \in\{1, \ldots, J\}$ and we define $t_{0}=0$. In the practice, credit events can occur at any point of the interval $\left[0, t_{J}\right], t_{J}=T$. For simplification we let the defaults occur in the midpoint of the two premium payment dates, i.e. $\left(t_{j}+t_{j+1}\right) / 2$. Then we let the random variable $\tau_{k}, k \in\{1, \ldots, K\}$, defined in the probability space $(\Omega, \mathcal{F}, \mathbb{P})$, be the default time of the $k$ th entity standing for the survival length and $\mathcal{R}$ be the constant recovery rate.

Then we can specify the default of the $k$ th entity by an indicator function $\mathbb{I}$ as follows,

$$
\Lambda_{k, t_{j}}=\mathbb{I}_{\left\{\tau_{k} \leq t_{j}\right\}}, k \in\{1, \ldots, K\}
$$

Then we give the portfolio loss process $L_{t_{j}}$ with the formulation as follows,

$$
L_{t_{j}}=\frac{1}{K} \sum_{k=1}^{K}(1-\mathcal{R}) \Lambda_{k, t_{j}}, j \in\{0, \ldots, J\} .
$$

We let $q \in\{1, \ldots, Q\}$ be the index of the $q$ th tranche and $L_{q, t_{j}}$ the tranche loss of the $q$ th tranche at $t_{j}$. And we define in this master thesis $A_{q}$ as the attachment point of the 
$q$ th tranche and $D_{q}$ as the detachment point. As the tranche loss is a function of the portfolio loss process, therefore we can present the $q$ th tranche loss as follows,

$$
L_{q, t_{j}}=\min \left\{\max \left\{L_{t_{j}}-A_{q}, 0\right\}, D_{q}-A_{q}\right\}, j \in\{1, \ldots, J\}, q \in\{1, \ldots, Q\}
$$

In the run of a CDS index tranche, if credit events of underlying entities occur then the premium to be payed in the next period need to be adjusted according to the outstanding notional $P_{q, t_{j}}$ which can be represented as follows,

$$
P_{q, t_{j}}=D_{q}-A_{q}-L_{q, t_{j}}
$$

Under the non-arbitrage assumption the expectation of the accumulative payments generated by the protection buyer and seller should be equal. In the CDO pricing study people use two terminologies for these two expectations, the default leg $\mathcal{D} \mathcal{L}_{q}$ which represents for the expectation of the aggregated compensation payments from the protection seller side, and the premium leg $\mathcal{P} \mathcal{L}_{q}$ which stands for the expectation of the aggregated premium payments from the protection buyer side. The formulation of the both can be shown as follows,

$$
\mathcal{D} \mathcal{L}_{q}=\mathbb{E}\left[\sum_{j=1}^{J} \eta_{t_{j}} N\left(L_{q, t_{j}}-L_{q, t_{j-1}}\right)\right], q \in\{1, \ldots, Q\}
$$

where $\eta_{t_{j}}$ is the discount function dependent on the survival length at each payment point and we use $S_{q}^{C D O}$ as the spread of the $q$ th tranche.

As in the market practice the protection buyer of an equity tranche needs to pay not only a fixed payment but also an upfront payment, therefore we give two formulas of the premium legs respectively for the equity tranche and the non-equity tranches in Formula (4.7) and Formula (4.6),

$$
\begin{gathered}
\mathcal{P} \mathcal{L}_{q}=\mathbb{E}\left[\sum_{j=1}^{J} \eta_{t_{j}} S_{q}^{C D O}\left(t_{j}-t_{j-1}\right) N\left(P_{q, t_{j}}+P_{q, t_{j-1}}\right) / 2\right], q \geq 2, \\
\mathcal{P} \mathcal{L}_{1}=\mathbb{E}\left[\left(D_{1}-A_{1}\right) N S_{1}^{C D O}-\sum_{j=1}^{J} 0.05 \eta_{t_{j}}\left(t_{j}-t_{j-1}\right) N\left(P_{1, t_{j}}+P_{1, t_{j-1}}\right) / 2\right],
\end{gathered}
$$


where in Formula (4.7) $S_{1}^{C D O}$ is the upfront payment rate and 500 is the basis point set in the quotation convention of the iTraxx Europe Series 8.

According to Formulas (4.5), (4.7) and (4.6), then under the non-arbitrage assumption we have,

$$
\mathcal{P} \mathcal{L}_{q}=\mathcal{D} \mathcal{L}_{q}
$$

then we can plug Formula (4.6) and Formula (4.5) into Formula (4.8) and obtain

$$
\mathbb{E}\left[\sum_{j=1}^{J} \eta_{t_{j}} S_{q}^{C D O}\left(t_{j}-t_{j-1}\right) N\left(P_{q, t_{j}}+P_{q, t_{j-1}}\right) / 2\right]=\mathbb{E}\left[\sum_{j=1}^{J} \eta_{t_{j}} N\left(L_{q, t_{j}}-L_{q, t_{j-1}}\right)\right]
$$

where $q \geq 2$. Hence the $q$ th CDS index tranche premium $S_{q}^{C D O}$ for $q \geq 2$ can be extracted as follows,

$$
\mathrm{S}_{q}^{C D O}=\frac{\mathbb{E}\left[\sum_{j=1}^{J} \eta_{t_{j}}\left(L_{q, t_{j}}-L_{q, t_{j-1}}\right)\right]}{\mathbb{E}\left[\sum_{j=1}^{J} \eta_{t_{j}}\left(t_{j}-t_{j-1}\right)\left(P_{q, t_{j}}+P_{q, t_{j-1}}\right) / 2\right]}
$$

Similarly to the routine in Formula (4.9) for $q=1$, the equity tranche, the CDS index tranche upfront payment rate $S_{1}^{C D O}$ can be given as follows,

$$
\left(D_{1}-A_{1}\right) S_{1}^{C D O} N-\mathbb{E}\left[\sum_{j=1}^{J} 0.05 \eta_{t_{j}}\left(t_{j}-t_{j-1}\right) N\left(P_{1, t_{j}}+P_{1, t_{j-1}}\right) / 2\right]=\mathcal{D} \mathcal{L}_{\mathfrak{l}}(4.10)
$$

therefor we can extract the $S_{1}^{C D O}$ from Formula (4.10) as follows,

$$
S_{1}^{C D O}=\mathbb{E}\left[\frac{\sum_{j=1}^{J} \eta_{t_{j}}\left\{\left(L_{1, t_{j}}-L_{1, t_{j-1}}\right)-0.05\left(t_{j}-t_{j-1}\right)\left(P_{1, t_{j}}+P_{1, t_{j-1}}\right) / 2\right\}}{D_{1}-A_{1}}\right] .
$$

According to the Table 3.3 we need to note that the 5 year CDS index equity tranche upfront payment rate $S_{1}^{C D O}=16.67$. Here 16.67 is the quoting convention in the Bloomberg terminal and actually with the upper formula for $S_{1}^{C D O}$ it is equal to 0.1667 $(=16.67 / 100)$ and $S_{q \geq 2}^{C D O}$ in the Table 3.3 is quoted as 106.42 , but with the Formula (4.9) it is equal to $0.010642(=106.42 / 10000)$. 


\subsection{Modeling of Defaults}

\section{Modeling of Marginal Default}

As mentioned at the beginning that $\tau_{k}, k \in\{1, \ldots, K\}$ is the random variable of survival length (or termed as the default time) of the $k$ th entity in the reference pool, then we can define $\tau_{k} \sim F_{k}$, i.e. $F_{k}$ is the CDF of $\tau_{k}$ such that

$$
F_{k}(t)=\mathbb{P}\left(\tau_{k} \leq t\right), t \geq 0
$$

Then the survival function $S_{k}$ can be given as follows,

$$
S_{k}(t)=1-\mathbb{P}\left(\tau_{k} \leq t\right)=1-F_{k}(t), t \geq 0 .
$$

In a bond market case we can understand the function $F_{k}(t)$ as the probability of a bond which can not survive over the length of $t$, while the survival function can be understood as the probability of a bond which can survive over the length of $t$.

Meanwhile we assume that the random variable $\tau_{k}$ has a PDF such that

$$
f_{k}(t)=F_{k}^{\prime}(t)=-S_{k}^{\prime}(t)=\lim _{\Delta \rightarrow 0^{+}} \frac{\mathbb{P}\left(t \leq \tau_{k} \leq t+\Delta\right)}{\Delta} .
$$

Then we define the hazard rate function $h_{k}(t)$ such that

$$
h_{k}(t)=\frac{f_{k}(t)}{1-F_{k}(t)}=\frac{-S_{k}^{\prime}(s)}{S_{k}(t)} .
$$

Then we can obtain the $S_{k}(t)$ by integrating the Formula (4.14) in both sides over the interval $[0, t]$ such that

$$
\int_{0}^{t} h_{k}(s) \mathrm{d} s=\int_{0}^{t} \frac{-S_{k}^{\prime}(s)}{S_{k}(s)} \mathrm{d} s .
$$


Then we continue to transform the both sides of the Formula (4.15) with the exponential function such that

$$
\begin{aligned}
\exp \left(\int_{0}^{t} h_{k}(s) \mathrm{d} s\right) & =\exp \left(\int_{0}^{t} \frac{-S_{k}^{\prime}(s)}{S_{k}(s)} \mathrm{d} s\right) \\
& =\exp \left(\ln S_{k}(0)-\ln S_{k}(t)\right) \\
& =\exp \left(\ln \frac{S_{k}(0)}{S_{k}(t)}\right) \\
& =\frac{1}{S_{k}(t)}
\end{aligned}
$$

After permutation we can have the following representation of the survival function $S_{k}(t)$

$$
S_{k}(t)=\exp \left(-\int_{0}^{t} h_{k}(s) \mathrm{d} s\right)
$$

According to the Formula (4.17) we can get the CDF representation of the $\tau_{k}$ as follows

$$
F_{k}(t)=1-S_{k}(t)=1-\exp \left(-\int_{0}^{t} h_{k}(s) \mathrm{d} s\right) .
$$

And for the simplicity we set in this work the hazard rate function as a constant $h$ for all $k$, therefore the CDF representation of the $\tau_{k} \in\{1, \ldots, K\}$ can be shown as

$$
F_{k}(t)=1-\exp (-h t)
$$

\section{Modeling of Joint Defaults}

As we already given in the Formula (4.1) that $\tau_{k}$ is a random variable for default time of the $k$ th entity and we have also defined $F_{k}(t)$ in the formula (4.11) as the CDF of $\tau_{k}$, but only the marginal CDF $F_{k}(t)$ is not enough to describe the behavior of default times in a portfolio as it is not rare that defaults can occur jointly, therefore we need to model the joint behavior of these default times, i.e. modeling for a random vector $\left(\tau_{1}, \ldots, \tau_{K}\right)^{\top}$. For modeling the behavior of default times $\left(\tau_{1}, \ldots, \tau_{K}\right)^{\top}$ we need a joint $\mathrm{CDF}$, for which a copula function can be employed. 
As shown in Formula (4.19) that

$$
F_{k}(t)=1-\exp (-h t)
$$

then we can derive that $\exp \left(-h \tau_{k}\right)$ is uniformly distributed over $[0,1]$, which can be given by the following lemma,

Lemma 4.1. Let $\tau_{k} \sim F_{k}, k \in\{1, \ldots, K\}$, where $F_{k}=\mathbb{P}\left(\tau_{k} \leq t\right)=1-\exp (-h t)$. Then $\exp \left(-h \tau_{k}\right) \sim$ Uniform $[0,1]$.

Proof. Let the random variable $W=\exp \left(-h \tau_{k}\right)$, then the CDF of $W$ can be represented as follows,

$$
\begin{aligned}
\mathbb{P}(W \leq w) & =\mathbb{P}\left(\exp \left(-h \tau_{k}\right) \leq w\right) \\
& =\mathbb{P}\left(-h \tau_{k} \leq \ln w\right) \\
& =\mathbb{P}\left(\tau_{k} \geq \ln -\frac{\ln w}{h}\right) \\
& =1-\mathbb{P}\left(\tau_{k} \leq \ln -\frac{\ln w}{h}\right) \\
& =1-\left(1-\exp \left(-h\left(-\frac{\ln w}{h}\right)\right)\right) \\
& =1-1+\exp (\ln w) \\
& =w .
\end{aligned}
$$

Therefore it can be easily proved that the function $\mathbb{P}(W \leq w)=w$ satisfies the definition and properties of the uniformly distributed CDF over $[0,1]$.

Then we define $U_{k}=\exp \left(-h \tau_{k}\right), k \in\{1, \ldots, K\}$ and $F_{k}^{U n i f}\left(u_{k}\right)$ as the marginal CDF of $U_{k}$, which is the CDF of a uniformly distributed random variable, then we can represent the joint $\mathrm{CDF}$ of $\left(U_{1}, \ldots, U_{K}\right)^{\top}$ such that

$$
\begin{aligned}
\mathbb{P}\left(U_{1} \leq u_{1}, \ldots, U_{K} \leq u_{K}\right) & =C\left(F_{1}^{U n i f}\left(u_{1}\right), \ldots, F_{K}^{U n i f}\left(u_{K}\right)\right) \\
& =C\left(u_{1}, \ldots, u_{K}\right)
\end{aligned}
$$


Then we can sample $\left(U_{1}, \ldots, U_{K}\right)^{\top}$ from the copula function $C\left(u_{1}, \ldots, u_{K}\right)$. As $U_{k}=$ $\exp \left(-h \tau_{k}\right), k \in\{1, \ldots, K\}$ then we can obtain that

$$
\tau_{k}=\frac{-\ln U_{k}}{h}
$$

Hence we can obtain

$$
\left(\tau_{1}, \ldots, \tau_{K}\right)^{\top}=\left(\frac{-\ln U_{1}}{h}, \ldots, \frac{-\ln U_{K}}{h}\right)^{\top}
$$

After performing $M$ times Monte Carlo simulations with the routine in the Formula (4.23) then we obtain samples $\left(z_{1}^{m}, \ldots, z_{K}^{m}\right)^{\top}, m \in\{1, \ldots, M\}$. Then we can calculate the empirical expectation of $\mathbb{E}\left[L_{q, t_{j}}\right], q \in\{1, \ldots, Q\}$ and $j \in\{1, \ldots, J\}$, such that

$$
\hat{\mathbb{E}}\left[L_{q, t_{j}}\right]=\frac{1}{M} \sum_{m=1}^{M}\left(\min \left\{\max \left\{\frac{1}{K} \sum_{k=1}^{K}(1-\mathcal{R}) \mathbb{I}_{\left\{\mathfrak{t}_{k}^{m} \leq t_{j}\right\}}-A_{q}, 0\right\}, D_{q}-A_{q}\right\}\right)
$$

Then we can plug $\widehat{\mathbb{E}}\left[L_{q, t_{j}}\right]$ into the Formula (4.5), (4.6) and (4.7) to obtain $\widehat{\mathcal{D L}}_{q}$ and $\widehat{\mathcal{P L}}_{q}$, therefore at last we can obtain empirical representations for spreads of CDS index tranches with the following formulas.

$$
\begin{gathered}
\widehat{S}_{1}^{C D O}=\widehat{\mathbb{E}}\left[\frac{\sum_{j=1}^{J} \eta_{t_{j}}\left\{\left(L_{1, t_{j}}-L_{1, t_{j-1}}\right)-0.05\left(t_{j}-t_{j-1}\right)\left(P_{1, t_{j}}+P_{1, t_{j-1}}\right) / 2\right\}}{D_{1}-A_{1}}\right], \\
\widehat{S}_{q \geq 2}^{C D O}=\frac{\widehat{\mathbb{E}}\left[\sum_{j=1}^{J} \eta_{t_{j}}\left(L_{q, t_{j}}-L_{q, t_{j-1}}\right)\right]}{\widehat{\mathbb{E}}\left[\sum_{j=1}^{J} \eta_{t_{j}}\left(t_{j}-t_{j-1}\right)\left(P_{1, t_{j}}+P_{1, t_{j-1}}\right) / 2\right]} .
\end{gathered}
$$

\section{Copula Choice}

In the first and second part of this section we have already introduced how to use copula to model the joint behavior of default times, then we need to face a question of choosing a copula. We need to consider three aspects of a copula: heterogeneous dependence structures between groups, non-elliptical distributions and asymmetrical tail-dependence. As lacking of these three aspects in the exchangeable Gaussian copula, the implied compound correlations under such copula show a so-called "correlation smile", which is illustrated in the Figure 4.1. 


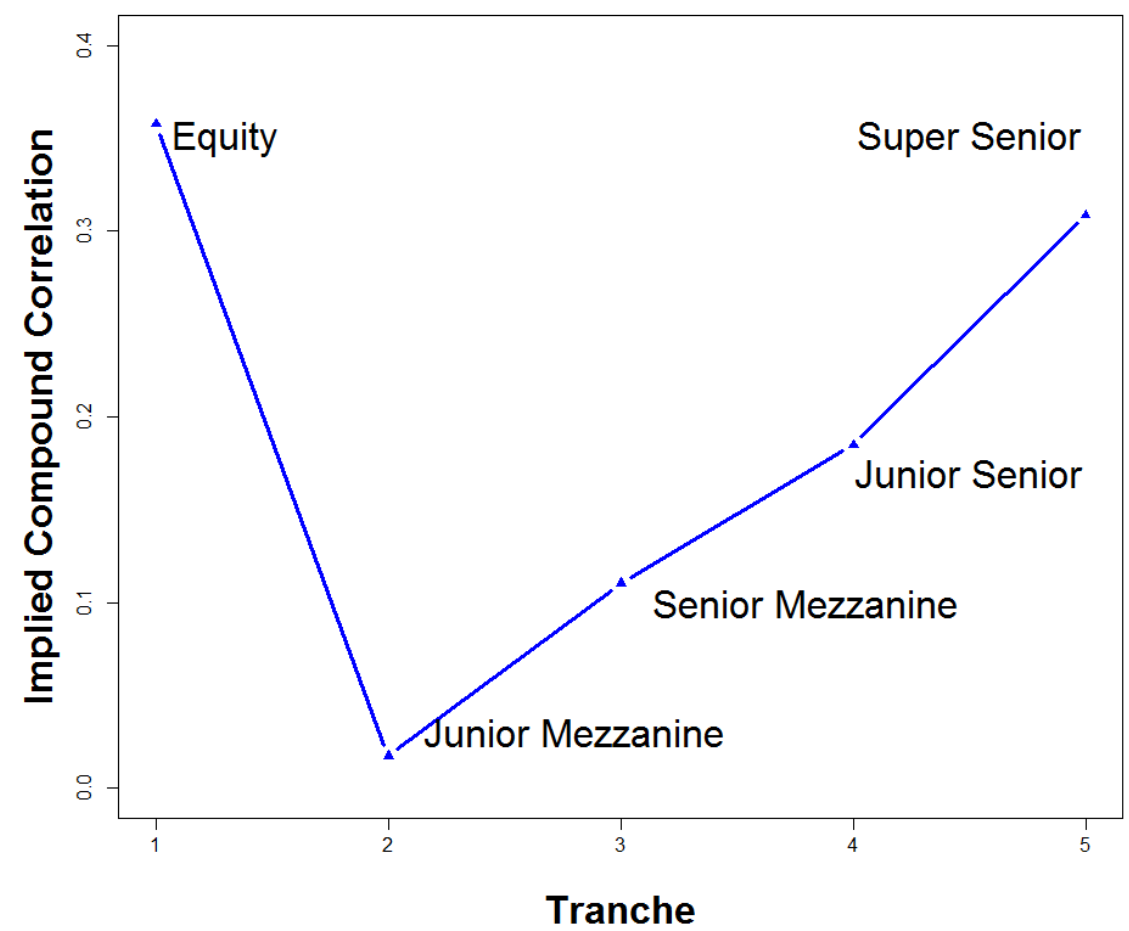

Figure 4.1: Implied correlation $\rho_{\mathcal{P}, q}, q \in\{1, \ldots, 5\}$ for 5 tranches of iTraxx Europe Series 8 on 2007-11-02 under the Gaussian copula.

QMARcorrsmile.R

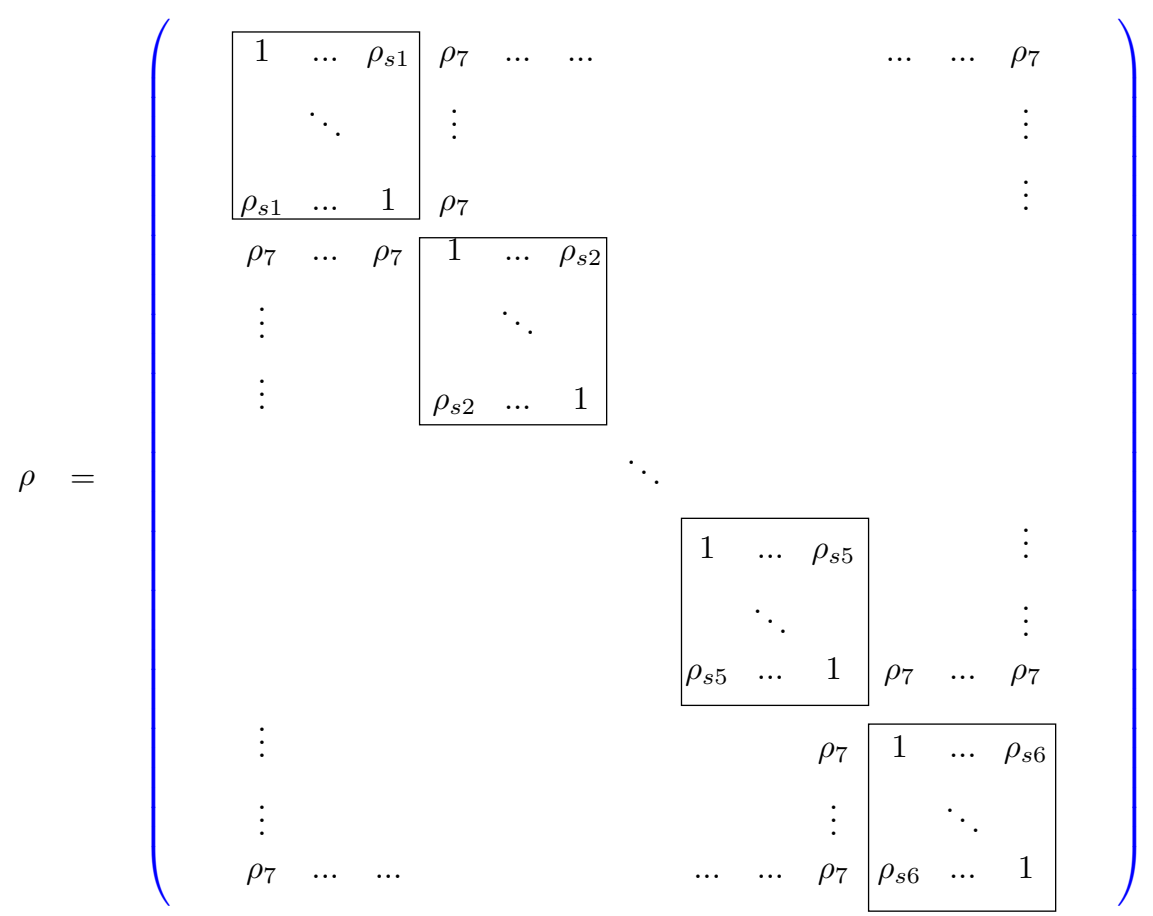

Figure 4.2: A Correlation Matrix owns 6 Sectors, where $\rho_{s i}, i \in\{1, \ldots, 6\}$ is the correlations of the $i$ th sector and $\rho_{7}$ is used to specify the dependence between 7 sectors. 
In order to solve the problems in an exchangeable Gaussian copula we need to choose copulas with better features. For the problem of the heterogeneity of dependence between sectors we can choose a non-exchangeable Gaussian copula, i.e. adding more parameters in the correlation matrix given in the Figure 4.2 but it can still not solve the asymmetrical tail-dependence problem. For the Student- $t$ copula which has the heavy tail dependence but it lacks capacity to solve the non-elliptical distribution problem. Therefore we can employ some members of the Archimedean family such as the Clayton copula, the Joe copula and the Gumbel copula, which can solve the asymmetrical taildependence problem but not the problem of the heterogeneity of dependence between sectors as the Archimedean copulas own individually single parameter. Then we can advance to the hierarchical Archimedean copula which can solve all the aforementioned problems. As a hierarchical Archimedean copula can be a component copula of a mixed copula, therefore we can also consider the mixed copula introduced in Section 2.5. For illustration of the differences of tail-dependence between copulas we show a comparison of 2-dimensional copula densities in Figure 4.3. 


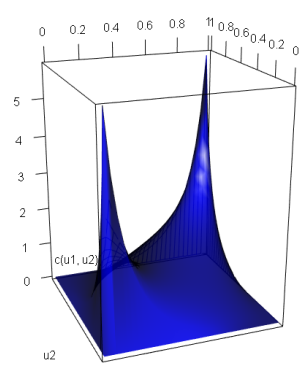

(a)

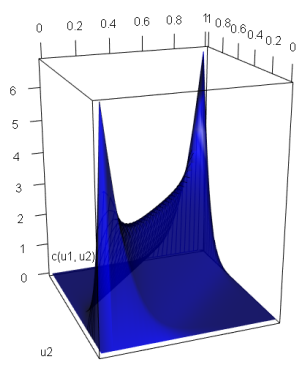

(c)

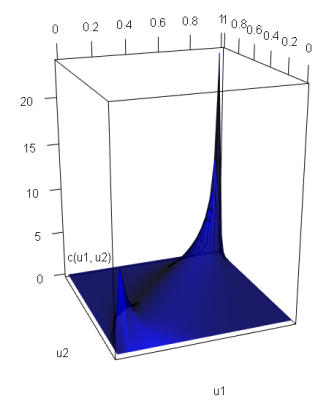

(e)

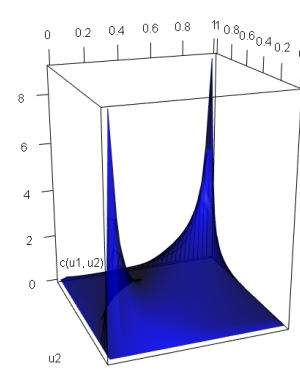

u1

(b)

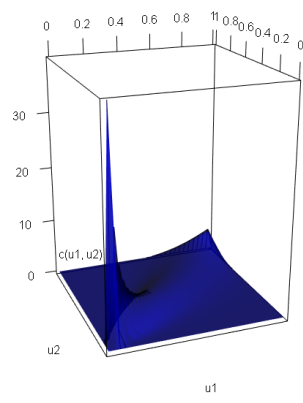

(d)

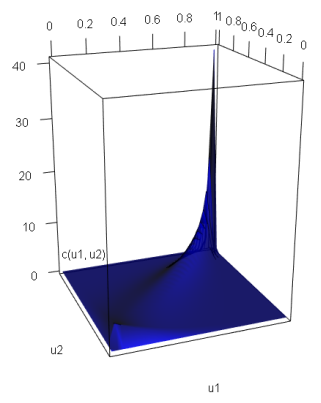

(f)

Figure 4.3: Copula density plots for 2-dimensional copulas with single parameters. From $(a)-(f)$ : (a) Gaussian copula, (b) Student- $t$ copula, (c) Frank copula, $(d)$ Clayton copula, $(e)$ Gumbel copula, $(f)$ Joe copula. For elliptical copulas the $\rho_{\mathcal{P}}=0.7$ and for Archimedean copulas the $\rho_{\mathcal{K}}=0.7$.

QMar2dpdf.R 


\section{Chapter 5}

\section{An Empirical Study}

\subsection{Data and Copula Models}

\section{Data Set}

In this empirical study part we employ the Markit iTraxx Europe Index Series 8 from the Bloomberg Terminal. As already introduced in Section 3.3, the iTraxx Europe index based tranches has four different maturity structures, 3, 5, 7 and 10 years and its underlying pool contains overall $K=125$ CDS contracts. Every six months the underlying pool will be updated for eliminating the already default entities. In this master thesis we choose the maturity with 5 years of the iTraxx Europe Series 8, which is issued on 2007.09.20 and ended on 2012.09.20, whose running period covers the financial crisis which is thought that CDOs are important triggers. And we do the pricing for all $Q=5 \mathrm{CDS}$ index tranches with 12 randomly chosen evaluation dates on 20071023, 20071102, 20071109, 20071206, 20080111, 20080204, 20080222, 20080318, 20080404, 20080407, 20080530, 20080701. We give the historical data of $Q=5$ CDS index tranches on these 12 pricing dates in Figure $5.1(a)$ and $(b)$. In the pricing we assume the risk-free rate as 0.03 and recovery rate as 0.40 which is consistent with it used in Markit company which administrates the Markit iTraxx Europe Index. 


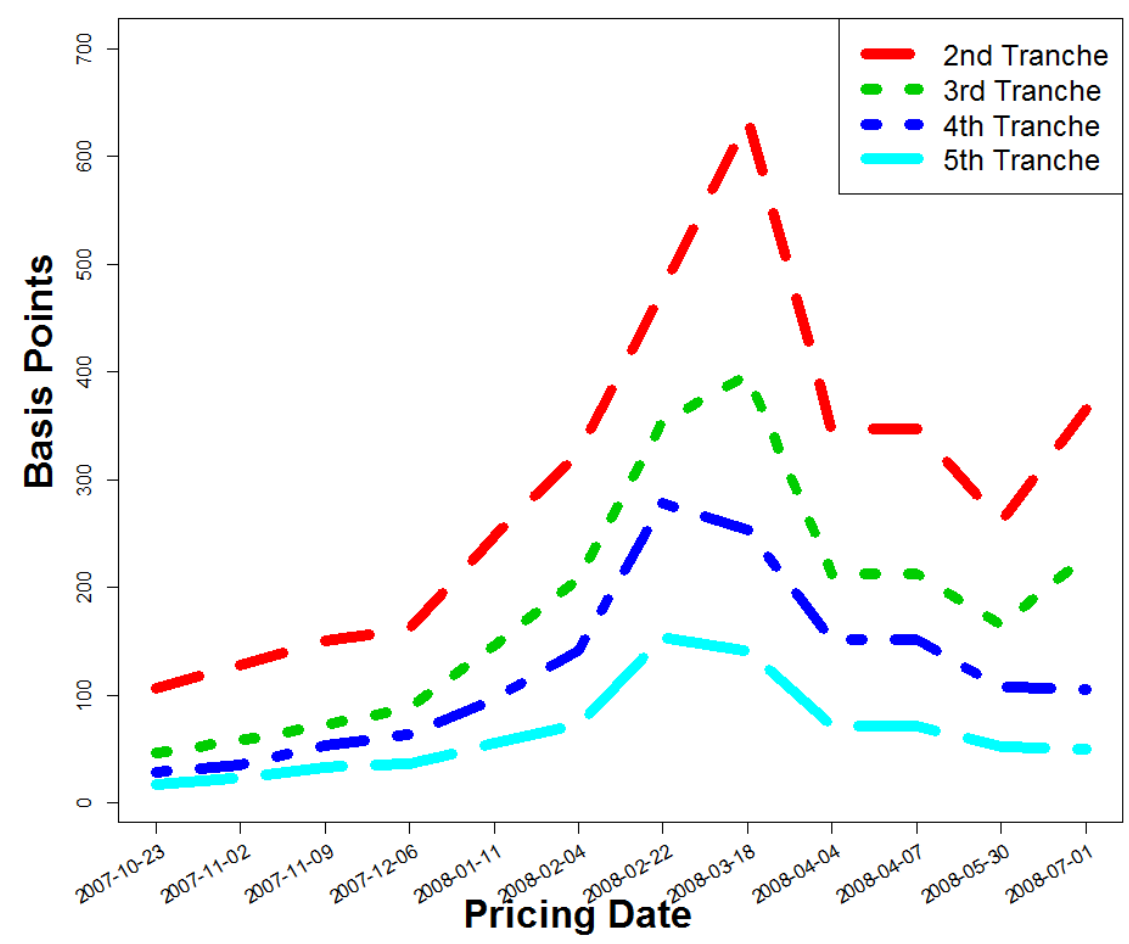

(a)

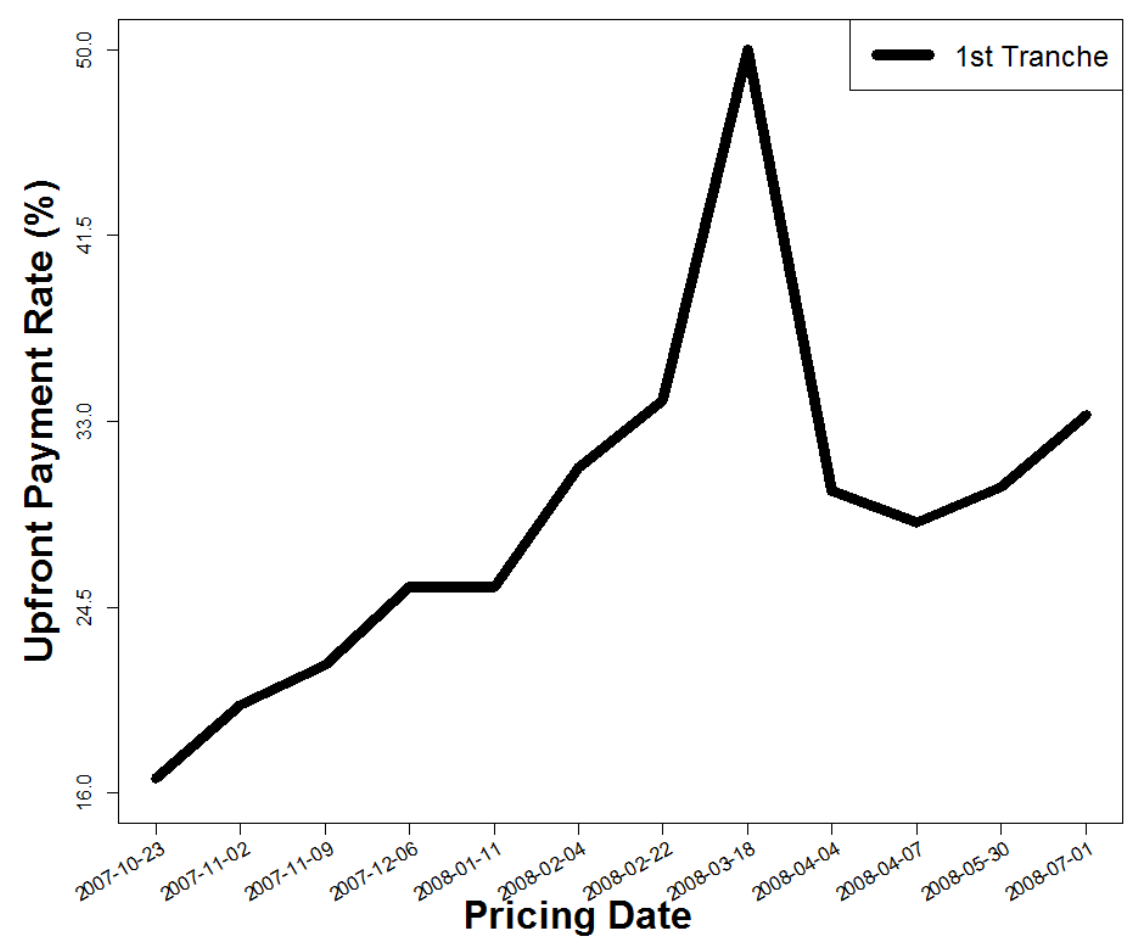

(b)

Figure 5.1: Tranche spreads at 12 pricing dates of Markit iTraxx Europe Index Series 8. (a) Tranche spreads for four tranches $(q=2,3,4,5)$ of Markit iTraxx Europe Index Series 8 from 20071023 to 20080701 by the Bloomberg Terminal. (b) Tranche spreads for equity tranche of Markit iTraxx Europe Index Series 8 from 20071023 to 20080701 by the Bloomberg Terminal.

Q MARtranchedata1.R Q MARtranchedata2.R 


\section{Models}

We have introduced copulas in Chapter 2 and CDS index tranche pricing in Chapter 4, therefore in this part we construct models for CDS index tranche pricing in the empirical study. We will use overall 43 copula models to be introduced as follows.

In the elliptical family of copulas we choose an exchangeable Gaussian copula and an exchangeable Student- $t$ copula such that

Model 1 :

$$
C\left(u_{1}, \ldots, u_{K} ; \theta\right)=C_{g s}\left(u_{1}, \ldots, u_{K} ; \theta\right)
$$

\section{Model 2:}

$$
C\left(u_{1}, \ldots, u_{K} ; \theta\right)=C_{t}\left(u_{1}, \ldots, u_{K} ; \theta, \mathrm{df}\right) .
$$

For Gaussian copula we also employ diverse dependence structures. In Model 3 represented in Formula (5.19) we use two parameters. $\rho_{2}$ is used for controlling the dependence within sector and $\rho_{1}$ is used to specify the dependence between sectors. The correlation matrix of Model 3 is written in Figure $5.2(a)$.

Model 3 :

$$
C\left(u_{1}, \ldots, u_{K} ; \theta\right)=C_{g s 1}\left(u_{1}, \ldots, u_{K} ; \rho_{g s 1}\right) .
$$

In Model 4 we let the recovery rate $\mathcal{R}$ be a random variable noted as $U_{K+1}$ shown in Formula (5.20) and uniformly distributed, then we set the parameter $\rho_{1}$ as the unique parameter for the dependence structure shown in Figure $5.2(b)$.

\section{Model 4:}

$$
C\left(u_{1}, \ldots, u_{K}, u_{K+1} ; \theta\right)=C_{g s 2}\left(u_{1}, \ldots, u_{K}, u_{K+1} ; \rho_{g s 2}\right)
$$

Model 5 is a generalization of Model 4 that we let the parameter $\rho_{2}$ specify the dependence within and between sectors. As the already added random variable $\mathcal{R}$ noted as $u_{K+1}$ in Formula (5.21) we let the parameter $\rho_{1}$ control the dependence structure 
between $U_{K+1}$ and $\left(U_{1}, \ldots, U_{K}\right)^{\top}$. The correlation matrix is illustrated in Figure 5.3 (a).

Model 5 :

$$
C\left(u_{1}, \ldots, u_{K}, u_{K+1} ; \theta\right)=C_{g s 3}\left(u_{1}, \ldots, u_{K}, u_{K+1} ; \rho_{g s 3}\right) .
$$

In Model 5 we use a single random recovery rate for all sectors but diverse sectors may have heterogeneous recovery rates, therefore in Model 6 we let $\left(U_{K+1}, \ldots, U_{K+6}\right)$ be six different uniform distributed random recovery rates. Then a new model can be obtained in Formula (5.22). In Figure $5.3(b)$ we show the correlation matrix for Model 5 that we use the parameter $\rho_{2}$ for within sector dependence and the parameter $\rho_{1}$ for between sectors dependence.

\section{Model 6 :}

$$
C\left(u_{1}, \ldots, u_{K}, u_{K+1}, \ldots, u_{K+6} ; \theta\right)=C_{g s 4}\left(u_{1}, \ldots, u_{K}, u_{K+1}, \ldots, u_{K+6} ; \rho_{g s 4}\right) .(5.6)
$$

In Model 7 referred to Formula (5.23) we still keep the six heterogeneous recovery rates setting but meanwhile we modify it that we let the parameter $\rho_{3}$ for specifying the dependence structure within sector and the parameter $\rho_{2}$ to control the dependence between $U_{s}, s \in\{K+1, \ldots, K+6\}$ and 6 sectors. At last the parameter $\rho_{1}$ is used to specify the dependence between blocks described in Figure $5.4(a)$.

\section{Model 7 :}

$$
C\left(u_{1}, \ldots, u_{K}, u_{K+1}, \ldots, u_{K+6} ; \theta\right)=C_{g s 5}\left(u_{1}, \ldots, u_{K}, u_{K+1}, \ldots, u_{K+6} ; \rho_{g s 5}\right) .(5.7
$$

In Model 8 we still use 3 parameters to specify the dependence structure of $\left(U_{1}, \ldots\right.$, $\left.U_{K}, U_{K+1}\right)^{\top}$. For the within sector dependence we embed the parameter $\rho_{3}$ and the parameter $\rho_{2}$ is used to control the between sectors dependence. At last the parameter $\rho_{1}$ is for the dependence between $U_{K+1}$, which stands for the single random recovery rate, and $\left(U_{1}, \ldots, U_{K}\right)^{\top}$. The correlation matrix is given in Figure $5.4(b)$. 


\section{Model 8 :}

$$
C\left(u_{1}, \ldots, u_{K}, u_{K+1} ; \theta\right)=C_{g s 6}\left(u_{1}, \ldots, u_{K}, u_{K+1} ; \rho_{g s 6}\right) .
$$

After models constructed by elliptical family of copula we give in the following the Archimedean copula based models. As introduced in Section 2.3 the Archimedean copula members share different tail-dependence structures. In Model 9 we use the Frank copula given in Formula (5.9), whose distribution shows an asymmetrical dependence and can be illustrated in Figure 2.2.

Model 9 :

$$
C\left(u_{1}, \ldots, u_{K} ; \theta\right)=C_{f}\left(u_{1}, \ldots, u_{K} ; \theta\right)
$$

Model 10 is a Clayton copula introduced in Section 2.3 and the model is given in Formula (5.10), which possesses features such as the left tail-dependence. In $\mathrm{Hu}$ [34] the Clayton copula is employed for the study of financial risk, which has shown that the joint crisis of stock markets can be modeled with the Clayton copula using its left tail-dependence.

Model 10 :

$$
C\left(u_{1}, \ldots, u_{K} ; \theta\right)=C_{c}\left(u_{1}, \ldots, u_{K} ; \theta\right) .
$$

In Section 2.3 we have introduced the Gumbel copula for its right tail-dependence and asymmetry which are not owned by an exchangeable Gaussian copula. Therefore we give the Gumbel copula model for multi-defaults dependence modeling represented in Formula (5.11).

\section{Model 11:}

$$
C\left(u_{1}, \ldots, u_{K} ; \theta\right)=C_{g}\left(u_{1}, \ldots, u_{K} ; \theta\right) .
$$

Model 12 is also an Archimedean copula, a Joe copula, which has right tail-dependence similar to the aforementioned Gumbel copula in Model 11. 


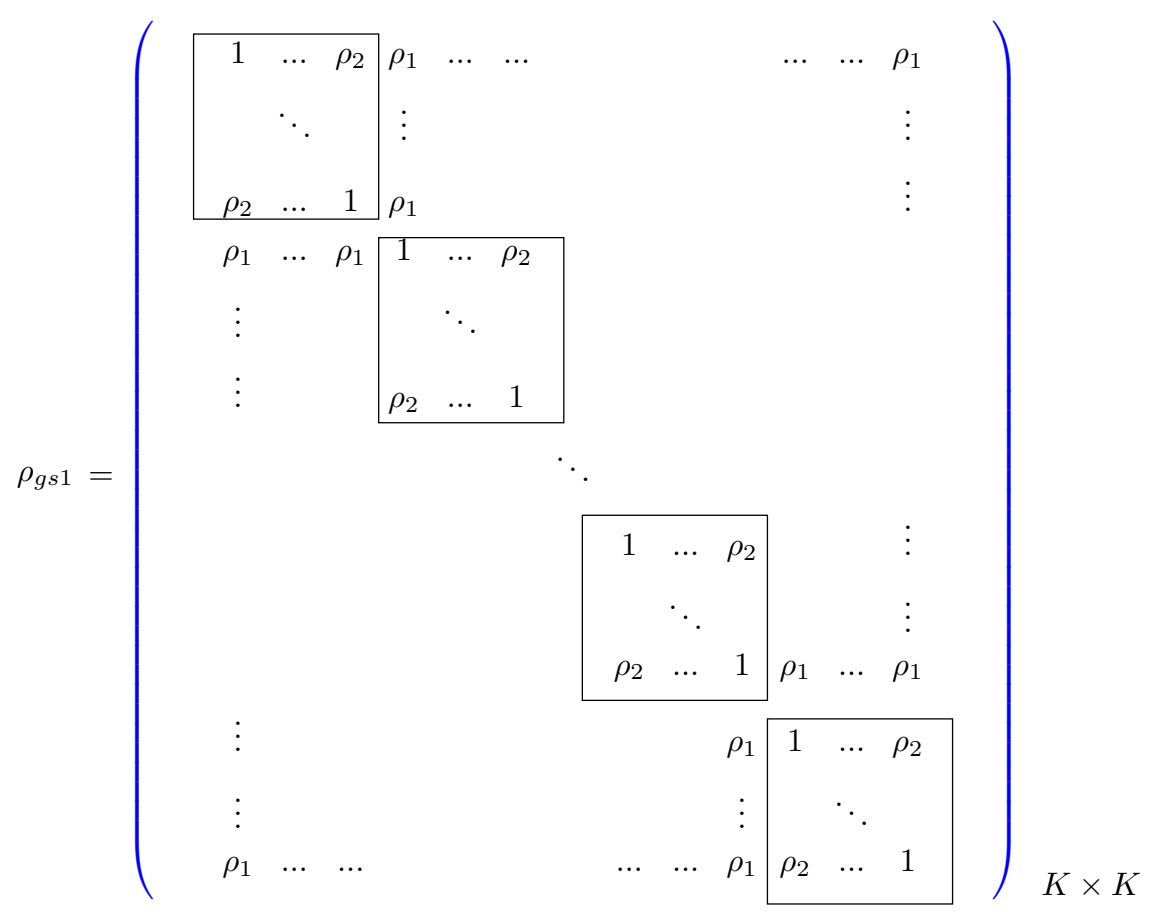

(a)

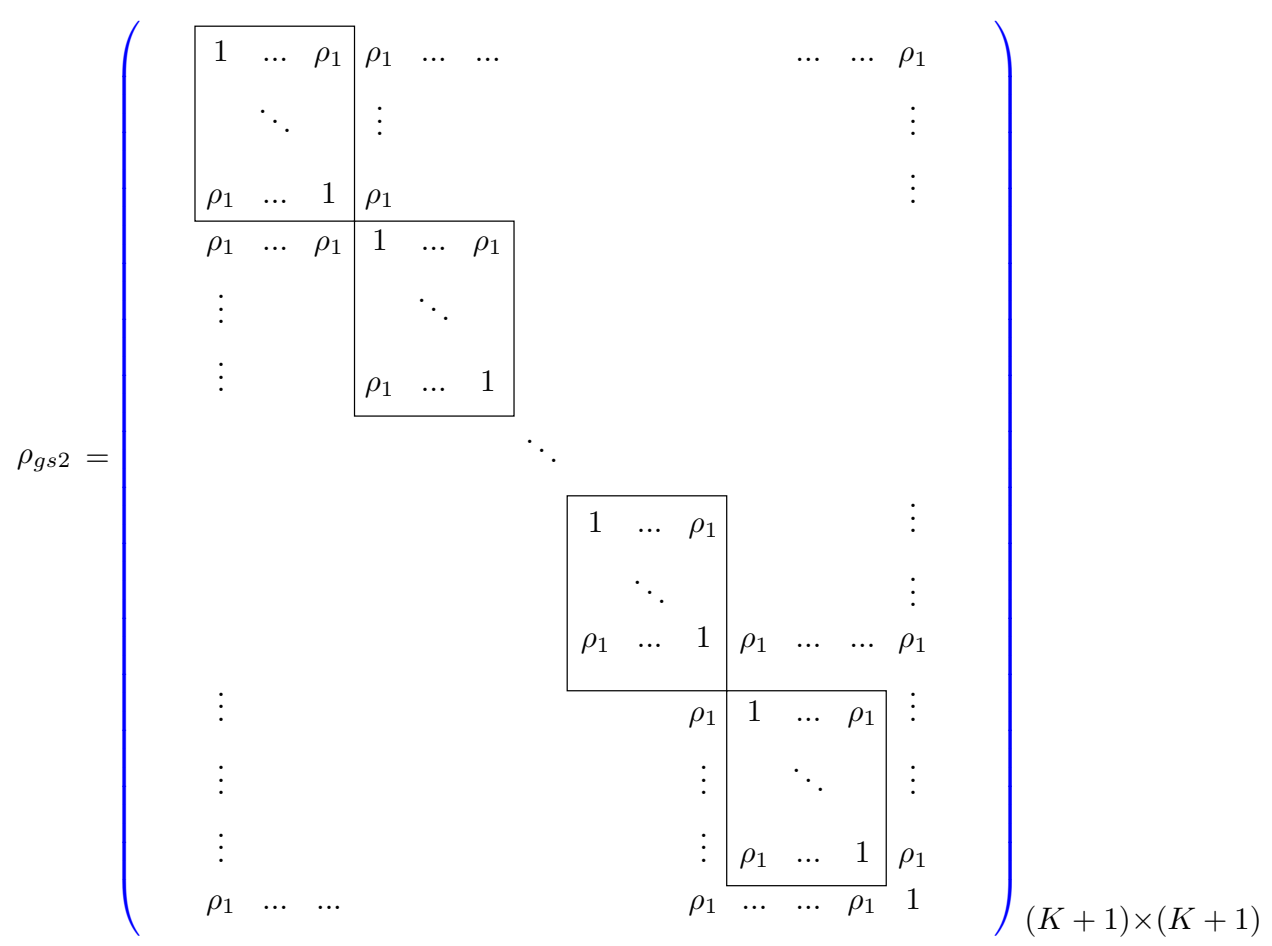

(b)

Figure 5.2: The structure of the correlation matrix $(a) \rho_{g s 1}$ is utilized in Model 3 and Model 38. And the structure of the correlation matrix $(b) \rho_{g s 2}$ is utilized in Model 4 and Model 39. 


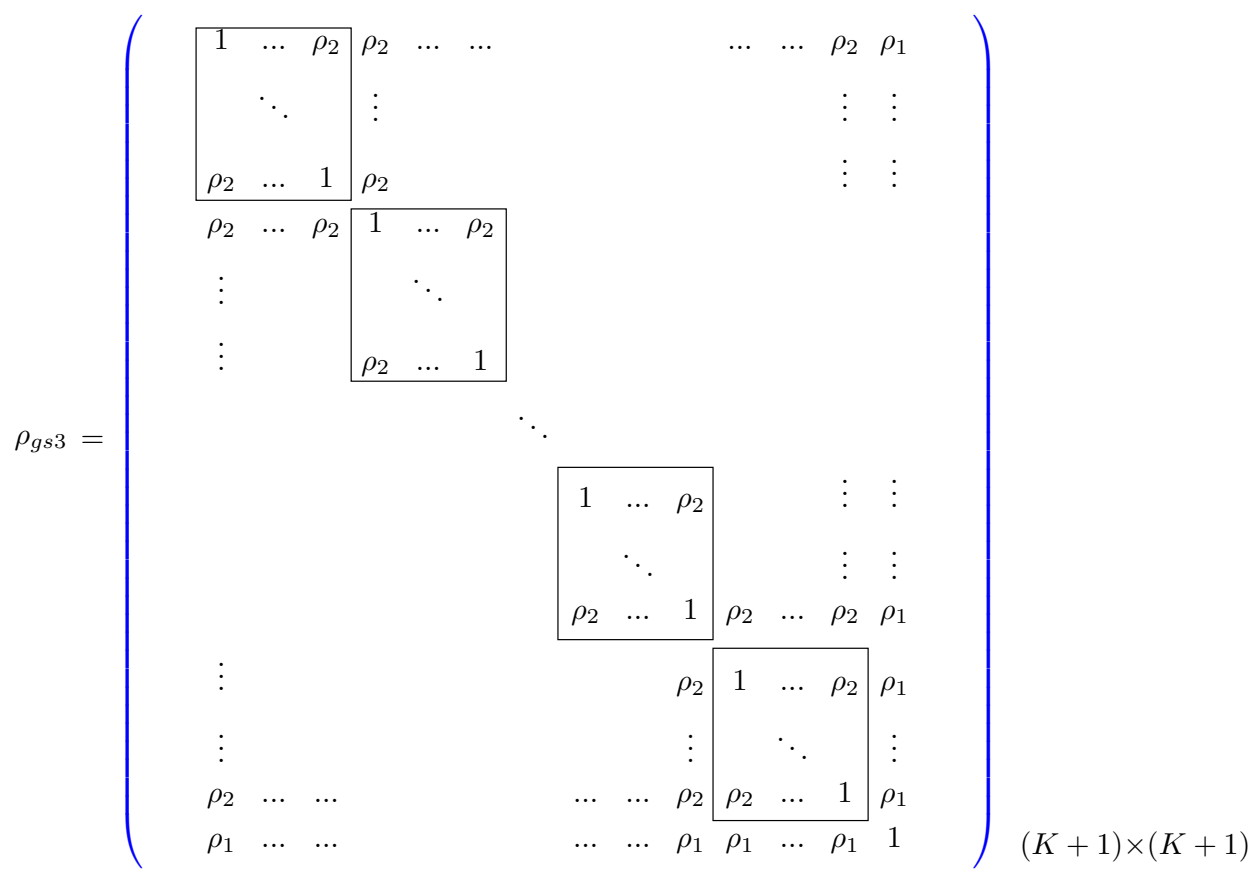

(a)
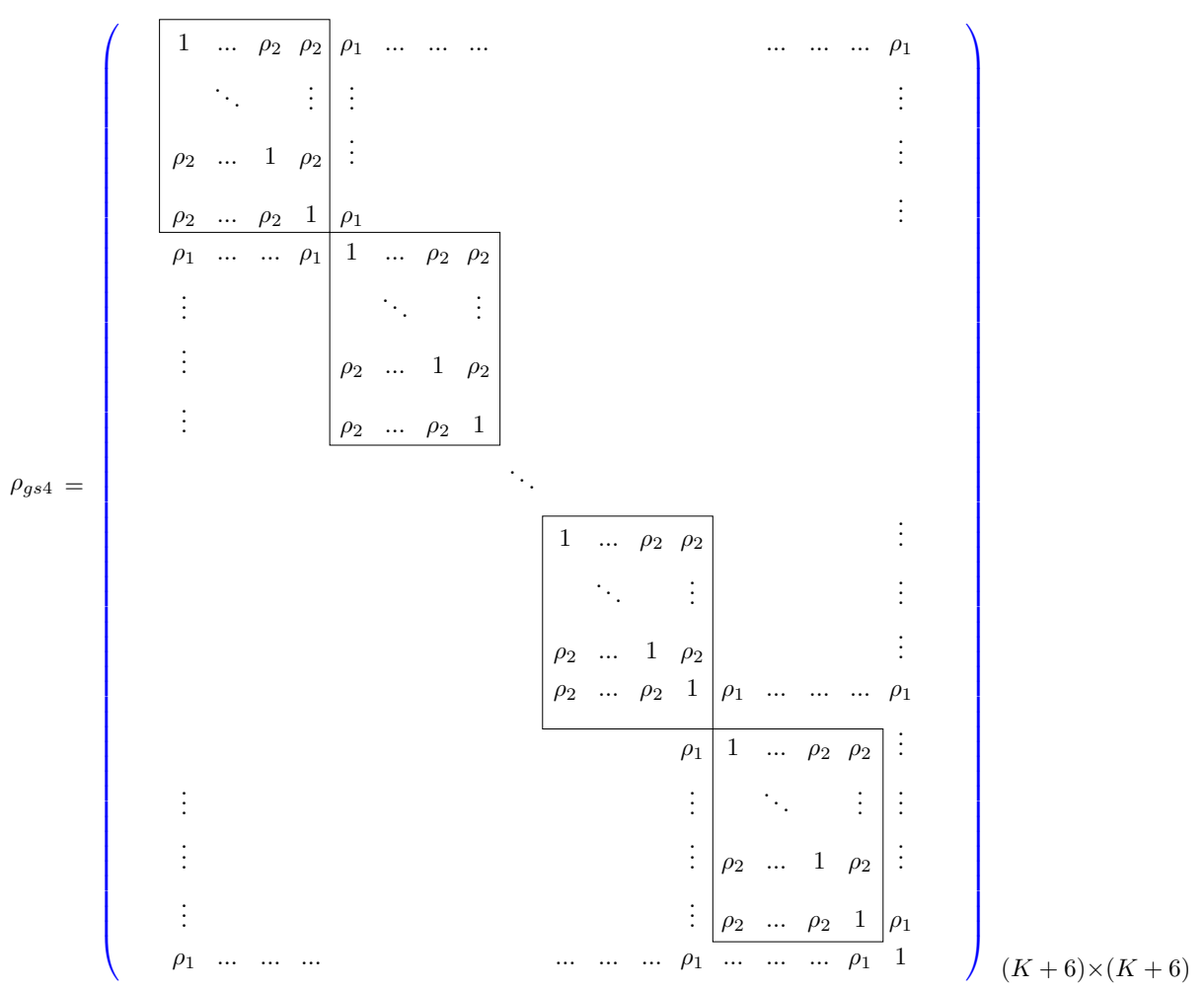

(b)

Figure 5.3: The structure of the correlation matrix $(a) \rho_{g s 3}$ is utilized in Model 5 and Model 40. And the structure of the correlation matrix $(b) \rho_{g s 4}$ is utilized in Model 6 and Model 41. 

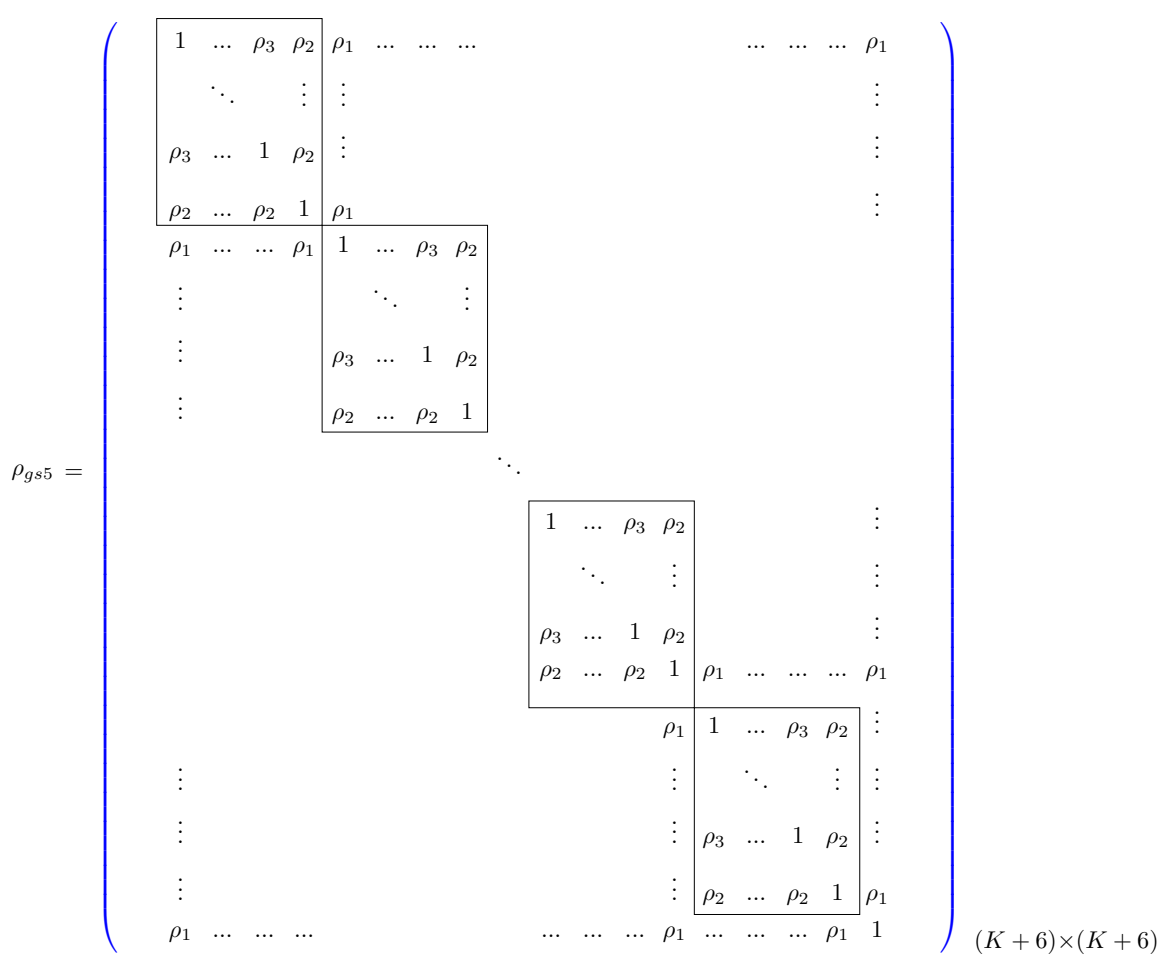

(a)
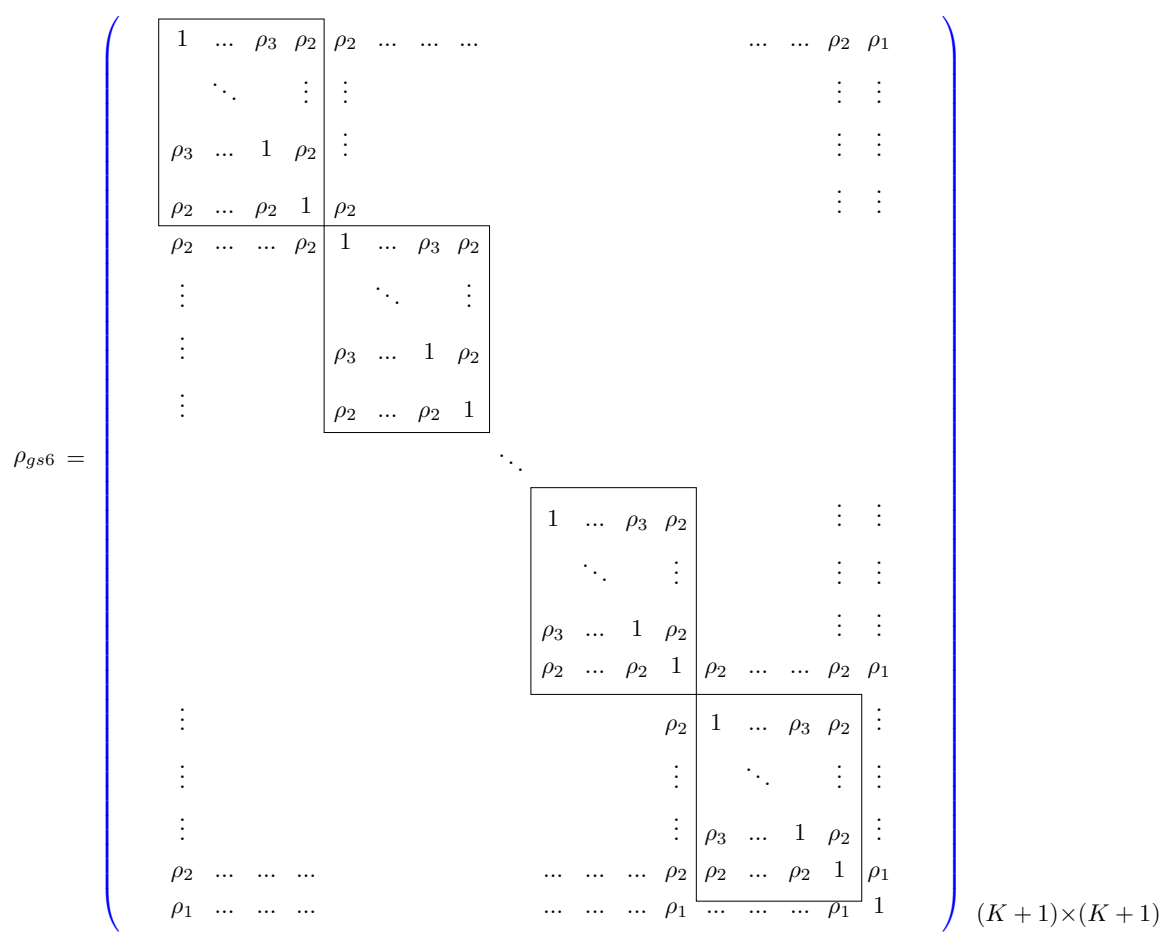

(b)

Figure 5.4: The structure of the correlation matrix $(a) \rho_{g s 5}$ is utilized in Model 7 and Model 42. And the structure of the correlation matrix $(b) \rho_{g s 6}$ is utilized in Model 8 and Model 43. 


\section{Model 12 :}

$$
C\left(u_{1}, \ldots, u_{K} ; \theta\right)=C_{j}\left(u_{1}, \ldots, u_{K} ; \theta\right)
$$

In Model 14, 15, 16 we introduce four hierarchical Archimedean copula models. As the hierarchical Archimedean copula model owns the two main features such as the heterogeneity of dependence between sectors and the asymmetrical tail-dependence so that it is appropriate to model the joint defaults behaviors.

Model 13 is a Gumbel copula with one parameter $\rho_{\mathcal{K} 1}$ for dependence within and between sectors. The random recovery $U_{K+1}$ is considered. The tree graph is illustrated in Figure $5.5(a)$ and the formula is given as follows,

\section{Model 13 :}

$$
C\left(u_{1}, \ldots, u_{K}, u_{K+1} ; \theta\right)=C_{n g 13}^{1}\left(u_{1}, \ldots, u_{K}, u_{K+1} ; \rho_{\mathcal{K} 1}\right),
$$

where $C_{n g 13}^{1}$ is the Gumbel copula. Therefore with Formula (5.13) the right taildependence has been taken account for the default times modeling.

Model 14 is a hierarchical Archimedean copula without random recovery using a root copula and a child copula. The parameter $\rho_{\mathcal{K} 2}$ is for dependence within a sector and $\rho_{\mathcal{K} 1}$ for dependence between sectors. The model is given in Formula (5.14) and the tree graph is illustrated in Figure $5.5(b)$.

\section{Model 14 :}

$$
\begin{aligned}
C\left(u_{1}, \ldots, u_{K} ; \theta\right)= & C_{n g 14}^{1}[ \\
& C_{n g 14}^{2}\left(u_{1}, \ldots, u_{s_{1}} ; \rho_{\mathcal{K} 2}\right) \\
& C_{n g 14}^{2}\left(u_{s_{1}+1}, \ldots, u_{s_{1}+s_{2}} ; \rho_{\mathcal{K} 2}\right), \ldots, \\
& C_{n g 14}^{2}\left(u_{s_{1}+\ldots+s_{5}+1}, \ldots, u_{K} ; \rho_{\mathcal{K} 2}\right) ; \rho_{\mathcal{K} 1} \\
& ],
\end{aligned}
$$

where $s_{i}, i \in\{1, \ldots, 6\}$ is the number of entities in $i$ th sector, $C_{n g 14}^{1}$ means the root copula in the hierarchical Archimedean copula with a Gumbel generator function as Model 14 and $C_{n g 14}^{2}$ means the child copula in this model. 
Model 15 is a generalization of Model 14 that six random recoveries are added in modeling, i.e. for each sector there is a responsible random recovery with uniform distribution. For Model 15 the Formula (5.15) is given and the tree graph is illustrated in Figure 5.5 $(c)$.

\section{Model 15 :}

$$
\begin{aligned}
C\left(u_{1}, \ldots, u_{K}, u_{K+1}, \ldots, u_{K+6} ; \theta\right)= & C_{n g 15}^{1}[ \\
& C_{n g 15}^{2}\left(u_{1}, \ldots, u_{s_{1}}, u_{K+1} ; \rho_{\mathcal{K} 2}\right), \\
& C_{n g 15}^{2}\left(u_{s_{1}+1}, \ldots, u_{s_{1}+s_{2}}, u_{K+2} ; \rho_{\mathcal{K} 2}\right), \ldots, \\
& C_{n g 15}^{2}\left(u_{s_{1}+\cdots+s_{5}+1}, \ldots, u_{K}, u_{K+6} ; \rho_{\mathcal{K} 2}\right) ; \rho_{\mathcal{K} 1} \\
& ] .
\end{aligned}
$$

Model 16 is a hierarchical Archimedean copula model with a Gumbel generator function using six random recoveries, $\left(U_{K+1}, \ldots, U_{K+6}\right)^{\top}$, and three parameters. $\rho_{\mathcal{K} 3}$ is utilized for within sector dependence, i.e. all six sectors share the same dependence parameter in every sector. $\rho_{\mathcal{K} 2}$ is employed for dependence between the $i$ th random recovery and the $i$ th sector, where $s \in\{1, \ldots, 6\}$. The parameter $\rho_{\mathcal{K} 1}$ control the dependence between the second layer child copulas, which can be shown in Formula (5.14). The main difference between Formula (5.13) and Formula (5.14) is that the six random recoveries are added into the Model 14 while there is only a single random recovery introduced in Model 13. And we illustrate the tree structure introduced in Section 2.4 for Model 16 in Figure 5.5 $(d)$.

\section{Model 16 :}

$$
\begin{aligned}
C\left(u_{1}, \ldots, u_{K}, u_{K+1}, \ldots, u_{K+6} ; \theta\right)= & C_{n g 16}^{1}\{ \\
& C_{n g 16}^{2}\left[u_{K+1}, C_{n g 16}^{3}\left(u_{1}, \ldots, u_{s_{1}} ; \rho_{\mathcal{K} 3}\right) ; \rho_{\mathcal{K} 2}\right], \\
& C_{n g 16}^{2}\left[u_{K+2}, C_{n g 16}^{3}\left(u_{s_{1}+1}, \ldots, u_{s_{1}+s_{2}} ; \rho_{\mathcal{K} 3}\right) ; \rho_{\mathcal{K} 2}\right], \ldots, \\
& C_{n g 16}^{2}\left[u_{K+6}, C_{n g 16}^{3}\left(u_{s_{1}+\cdots+s_{5}+1}, \ldots, u_{K} ; \rho_{\mathcal{K} 3}\right) ; \rho_{\mathcal{K} 2}\right] ; \rho_{\mathcal{K} 1} \\
& \} .
\end{aligned}
$$

Next we give the mixed copula models from Model 17 to Model 37. In a mixed copula 


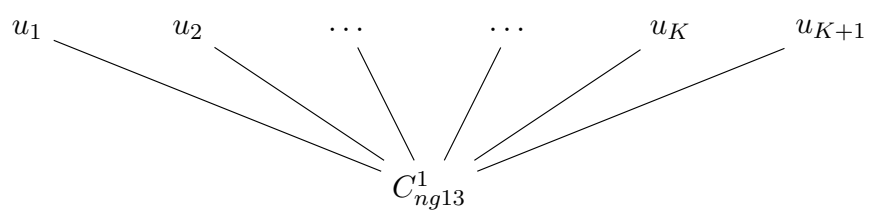

(a)
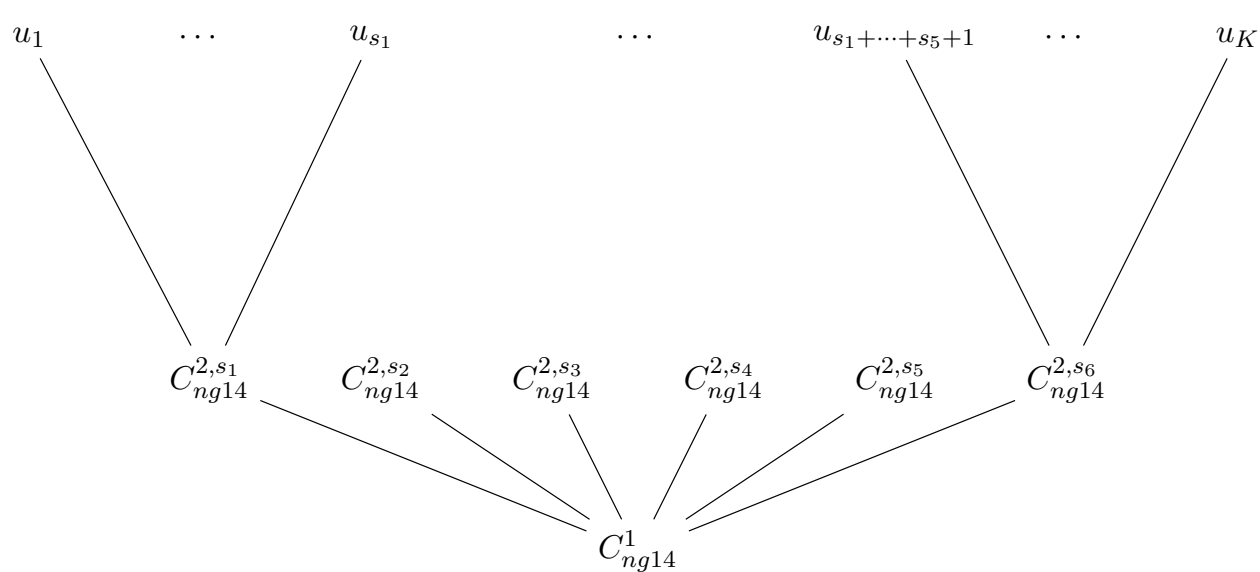

(b)

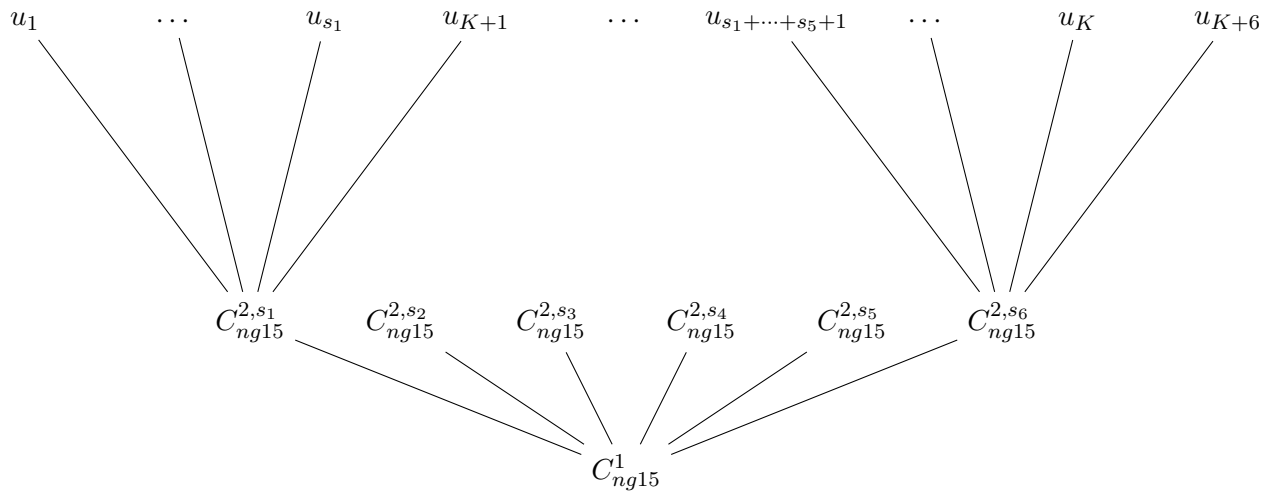

(c)
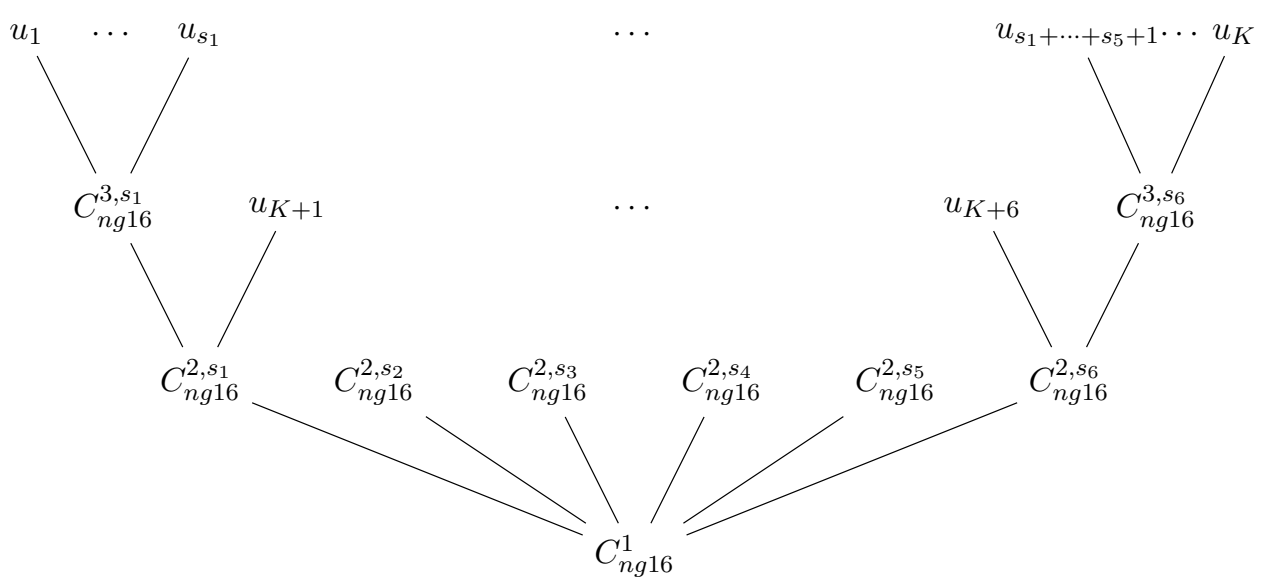

(d)

Figure 5.5: Tree structure plots for Model 13 in (a), Model 14 in (b), Model 15 in (c) and Model 16 in $(d)$. 
model we employ six copula models as the component copulas containing the exchangeable Gaussian copula, the Student- $t$ copula with degree of freedom equal to 3, the Frank copula, the Clayton copula, the Gumbel copula and the Joe copula. These six copula models are corresponding to Model 1, 2, 9, 10, 11, 12, i.e. two models from the elliptical family and four models from the Archimedean family. We define firstly some notations that we use lower letters $g s, t, f, c, g$ and $j$ to stand for correspondingly the exchangeable Gaussian copula, the Student- $t$ copula with degree of freedom equal to 3 , the Frank copula, the Clayton copula, the Gumbel copula and the Joe copula. And we set $w_{l}, l \in\{1,2\}$ as the weight for the $l$ th component copula, then a general formula for mixed copula models to be used in this master thesis can be given as follows,

$$
\begin{aligned}
C_{\text {compent } 1 \text {-compent } 2}\left(u_{1}, \ldots, u_{K} ; \theta\right)= & w_{1} C_{\text {compent } 1}\left(u_{1}, \ldots, u_{K} ; \theta_{1}\right) \\
& +w_{2} C_{\text {compent } 2}\left(u_{1}, \ldots, u_{K} ; \theta_{2}\right),
\end{aligned}
$$

where the compent 1 and the compent $\in\{g s, t, f, c, g, j\}$ and parameters $\theta_{1}$ and $\theta_{2}$ belong correspondingly to the component copula 1 and 2 . Let us see an example that Model 17 is a mixed Gaussian copula model with formula as follows,

\section{Model 17 :}

$$
C_{g s-g s}\left(u_{1}, \ldots, u_{K} ; \theta\right)=w_{1} C_{g s}\left(u_{1}, \ldots, u_{K} ; \theta_{1}\right)+w_{2} C_{g s}\left(u_{1}, \ldots, u_{K} ; \theta_{2}\right) .
$$

According to the convention in Formula (5.17), $C_{g s-g s}$ in Model 17 means that this model is constructed by mixture of two Gaussian $(g s)$ copulas. We give Model 17 to Model 37 in the following Table 5.1.

As the Gaussian copula has no tail-dependence, therefore we employ another member of elliptical copula with the fat tail-dependence feature, the Student- $t$ copula, for comparison of the pricing performance. At first we use the 12 degrees of freedom of the Student- $t$ copula in Model 2 and meanwhile we use the correlation structure same as the structure utilized in Model 3, 4, 5, 6, 7, 8, for which the correlation matrix can be referred in Figure 5.2, 5.3, 5.4.

\section{Model 38 :}

$$
C\left(u_{1}, \ldots, u_{K} ; \theta\right)=C_{t 38}\left(u_{1}, \ldots, u_{K} ; \rho_{t 38}\right)
$$


Model 39 :

$$
C\left(u_{1}, \ldots, u_{K}, u_{K+1} ; \theta\right)=C_{t 39}\left(u_{1}, \ldots, u_{K}, u_{K+1} ; \rho_{t 39}\right) .
$$

Model 40 :

$$
C\left(u_{1}, \ldots, u_{K}, u_{K+1} ; \theta\right)=C_{t 40}\left(u_{1}, \ldots, u_{K}, u_{K+1} ; \rho_{t 40}\right) .
$$

Model 41 :

$$
C\left(u_{1}, \ldots, u_{K}, u_{K+1}, \ldots, u_{K+6} ; \theta\right)=C_{t 41}\left(u_{1}, \ldots, u_{K}, u_{K+1}, \ldots, u_{K+6} ; \rho_{t 41}\right)(5
$$

Model 42 :

$$
C\left(u_{1}, \ldots, u_{K}, u_{K+1}, \ldots, u_{K+6} ; \theta\right)=C_{t 42}\left(u_{1}, \ldots, u_{K}, u_{K+1}, \ldots, u_{K+6} ; \rho_{t 42}\right)(5
$$

Model 43 :

$$
C\left(u_{1}, \ldots, u_{K}, u_{K+1} ; \theta\right)=C_{t 43}\left(u_{1}, \ldots, u_{K}, u_{K+1} ; \rho_{t 43}\right) .
$$

Here for all the Student- $t$ copula models employed in this master thesis we need to note the degrees of freedom that the Model 2 uses 12 different degrees of freedom, and the mixed copula models like the Model 18 and Model 23 to Model 27 use the degree of freedom equal to 3 , and the Model 38 to Model 43 use the 12 calibrated degrees of freedom by the Model 2.

For an overview of all 43 copula models used in this master thesis we give the following Table 5.1, where the index with the blue color means this model has been studied in the paper Hofert and Scherer [8] and the index with the red color means this model has been studied in the paper Choros-Tomczyk et al. [10]. And the Model 3 and Model 14 with the blue index and the red notation mean the both have been investigated in Hofert and Scherer [8] and Choros-Tomczyk et al. [10]. Hofert and Scherer [8]. 


\begin{tabular}{l|l|l|l|l|l|l|l}
\hline \hline Model & Notation & Model & Notation & Model & Notation & Model & Notation \\
\hline 1 & $C_{g s}$ & 12 & $C_{j}$ & 23 & $C_{t-t}$ & 34 & $C_{c-j}$ \\
2 & $C_{t}$ & 13 & $C_{n g 13}$ & 24 & $C_{t-f}$ & 35 & $C_{g-g}$ \\
3 & $C_{g s 1}$ & 14 & $C_{n g 14}$ & 25 & $C_{t-c}$ & 36 & $C_{g-j}$ \\
4 & $C_{g s 2}$ & 15 & $C_{n g 15}$ & 26 & $C_{t-g}$ & 37 & $C_{j-j}$ \\
5 & $C_{g s 3}$ & 16 & $C_{n g 16}$ & 27 & $C_{t-j}$ & 38 & $C_{t 38}$ \\
6 & $C_{g s 4}$ & 17 & $C_{g s-g s}$ & 28 & $C_{f-f}$ & 39 & $C_{t 39}$ \\
7 & $C_{g s 5}$ & 18 & $C_{g s-t}$ & 29 & $C_{f-c}$ & 40 & $C_{t 40}$ \\
8 & $C_{g s 6}$ & 19 & $C_{g s-f}$ & 30 & $C_{f-g}$ & 41 & $C_{t 41}$ \\
9 & $C_{f}$ & 20 & $C_{g s-c}$ & 31 & $C_{f-j}$ & 42 & $C_{t 42}$ \\
10 & $C_{c}$ & 21 & $C_{g s-g}$ & 32 & $C_{c-c}$ & 43 & $C_{t 43}$ \\
11 & $C_{g}$ & 22 & $C_{g s-j}$ & 33 & $C_{c-g}$ & & \\
\hline \hline
\end{tabular}

TABle 5.1: Abbreviations: $g s$ : Gaussian, $t$ : Student- $t, f$ : Frank, $c$ : Clayton, $g$ : Gumbel, $j$ : Joe, gsi, $i \in\{1, \ldots, 6\}$ : Gaussian with the correlation matrix $\rho_{g s i}, i \in$ $\{1, \ldots, 6\}, t j, j \in\{38, \ldots, 43\}$ : Student- $t$ with the same correlation matrix structure as $\rho_{g s i}, i \in\{1, \ldots, 6\}, n g$ : hierarchical Archimedean copula with the Gumbel generator function. Hofert (2008), Choros-Tomczyk, Härdle, Okhrin (2013).

\subsection{Parameter Calibration}

We have introduced hierarchical Archimedean copulas, Archimedean copulas, mixed copulas and elliptical copulas (HAME) in Chapter 2, which can be applied in CDS index tranche pricing by using the copula to construct the dependence structure of default times $\left(\tau_{1}, \ldots, \tau_{K}\right)^{\top}$ and Formula (4.18) to model the marginal CDF of $\tau_{k}, k \in$ $\{1, \ldots, K\}$. In this master thesis we assume the hazard function as a constant scalar $h$ and we imply this quantity from the market spreads of the iTraxx Europe Index Series 8. For a detailed method of implication of $h$ we refer to Hofert and Scherer [8].

At first step we choose a copula model listed in the Table 5.1 and then we sample $M=10^{4}$ runs according to $\left(U_{1}, \ldots, U_{K}\right)^{\top} \sim C$ to obtain samples $\left(u_{m, 1}, \ldots, u_{m, K}\right)^{\top}$, where $m$ is the runs of Monte Carlo simulation and $m \in\{1, \ldots, M\}$ to compute Formula (4.5), (4.6) and (4.7). Especially for models embedded with one random recovery such as Formula (5.20), (5.21), (5.24), (5.13), and with six random recoveries such as Formula (5.22), (5.23), (5.15), (5.16) we need to sample respectively according to $\left(U_{1}, \ldots, U_{K}, U_{K+1}\right)^{\top} \sim C$ and $\left(U_{1}, \ldots, U_{K}, U_{K+1}, \ldots, U_{K+6}\right)^{\top} \sim C$ to obtain samples. In this master thesis totally we employ 43 models listed in Table 5.1 containing 14 elliptical copulas from Model 1 to Model 8 and from Model 38 to Model 43, 5 Archimedean 
copulas from Model 9 to Model 13, 3 hierarchical Archimedean copulas from Model 14 to Model 16 and 21 mixed copulas from Model 23 to Model 37.

After $\left(U_{1}, \ldots, U_{K}\right)^{\top} \sim C$ sampled from copulas we can use Formula (4.22) to obtain the default times $\left(\tau_{1}, \ldots, \tau_{K}\right)^{\top}$ which can be utilized to compute the portfolio loss in Formula (4.2), $q$ th tranche loss in Formula (4.3) and the outstanding notional in Formula (4.4). At last by Formulas (4.5), (4.6) and (4.7) we can obtain the $q$ th default leg $\mathcal{D} \mathcal{L}_{q}$ and the $q$ th premium leg $\mathcal{P} \mathcal{L}_{q}$ for CDS index tranche pricing. Here we set the notation $\widehat{S}_{q}^{C D O}$, defined under Formula (4.25) and (4.26), as the tranche spreads by Monte Carlo simulations under models listed in Table 5.1 and $S_{q}^{\text {Market-CDO }}$ as the real market tranche spreads. And for the parameter calibration we use the following measure, which is a relative difference measure (RDM), similar to the formula introduced in Choros-Tomczyk et al. [10] such that,

$$
R D M=\min \sum_{q=1}^{Q} \frac{\left|\widehat{S}_{q}^{C D O}-S_{q}^{\text {Market-CDO }}\right|}{S_{q}^{\text {Market }-C D O}} .
$$

As we have seen that Formula (5.25) is an argument representation, therefore we need to perform numerical optimization to calibrate parameters. In Table 5.1 Model 1, 4, 9, 10, 11, 12, 13, 39 are single parameter models and Model 2, 3, 5, 6, 14, 15, 38, 40, 41 have 2 parameters. The rest Model 7, 8 and 16 to 37, 42 to 43 have 3 parameters. For all these models we employ the grid search with the multi-core parallel computation in the optimization, therefore all calibrated parameters are not local but global optima.

\subsection{Empirical Results and Analysis}

In this section we give the empirical results of the iTraxx Europe Series 8 index tranche pricing. In Table 5.2 and 5.3 we show the computation results according to the relative difference measure introduced in Formula (5.25). And in Table 5.4, 5.5, 5.6, 5.7 and 5.8 we show the calibrated parameters under the approach given in Section 5.2. In Table 5.9 we have calculated the mean of the relative difference measures based on 12 pricing days and a ranking based on the mean of the relative difference measures is given.

We find in Table 5.9 that according to the mean of the relative difference measure (RDM) introduced in Formula (5.25) the top three best performed models are correspondingly 
$C_{g-j}, C_{g-g}$ and $C_{f-j}$, whose RDMs graph can be seen in Figure $5.7(a)$. And we can find that the top fourteen models are all mixed copula models. Especially we can see top five models are not only the mixed models but also their components are all from the Archimedean family and it is quite clear that if a model want to be a member in top ten rank then there must be at least a component copula coming from a Gumbel copula or a Joe copula, which are both right tail-dependent. In the model list in Table 5.1 we know that Model 3, 6, 7, 8, Model 14 to Model 16, Model 38, 41, 42, 43 have specified for the heterogeneous dependence between sectors and mixed copula does not use a special parameter to do the same thing but the empirical results show that the mixed copula do consider these heterogeneity of dependence between sectors.

In the ranking in Table 5.9 another result is that the elliptical copulas perform worst. We can see that the worst ten models are almost elliptical copulas. For the worst models we have illustrated with Figure $5.7(b)$ and a comparison for the three worst performed models and the three best performed models are shown in Figure $5.6(a)$. And under the same structures we have compared the Gaussian copula models and the Student- $t$ copula models pair by pair, and we find that in every structure introduced by Figure $5.2,5.3,5.4$ the Student- $t$ copula models outperform the Gaussian copula models.

Hierarchical Archimedean copulas perform better than elliptical copulas. The best Hierarchical Archimedean copula model is $C_{n g 14}$ ranked at the 16 th place being not better than the best single parameter Archimedean copula $C_{j}$. And the best performed elliptical copula is $C_{t 43}$ ranking at the 25 th place. The last column in Table 5.9 shows that the elliptical copulas are not appropriate for modeling the defaults dependence under the iTraxx Europe index tranche context as we can see that the Frank copula, the Gaussian copula and the Student- $t$ copula rank in low place.

Another interesting result in Table 5.6 shows that the calibrated parameter $w_{1}$, which is the weight of the first component copula in a mixed copula model, gives a much larger quantity in a mixed copula composing an elliptical copula and a Gumbel copula or a Joe copula to the Gumbel or Joe copula, i.e. the calibration automatically chooses Gumbel or Joe rather than an elliptical copula, which means Gumbel and Joe copulas are appropriate for modeling default times of entities of the iTraxx Europe index components. The main reason is that the joint default times have a right tail-dependence. And from the results of parameters in Table 5.6 we can verify that the joint defaults are not left 
tail-dependent as the $w_{1}$ in model $C_{c-g}$ and the model $C_{c-j}$, which are correspondingly the mixed copula of a Clayton copula and a Gumbel or Joe copula, in 12 pricing days are mostly lower than 0.5, which can be an evidence of non-left-dependence. And another evidence is that the model $C_{c}$ performs the worst under the mean of RDM.

Therefore we can from the above analysis arrived at some conclusions. Firstly, the mixed copula model is superior against elliptical copulas, single parameter Archimedean copulas and 4 hierarchical Archimedean copulas employed in Table 5.1 according to the relative difference measure. Secondly, among the well performed mixed copula models the model employing a Gumbel or Joe copula has better performance as the both share the right tail-dependence. Thirdly, the joint default times obey a right tail-dependence not a left one and an elliptical one, therefor the Clayton copula and the Frank copula is not appropriate for modeling the joint defaults under the iTraxx Europe index tranche context. At last we conclude that the elliptical copulas are not appropriate for the CDS index tranche pricing as its elliptical distribution and symmetrical tail-dependence. In order to compare the performance of the 43 copula models, in Figure $5.6(b)$ we give the RDMs of the 43 models at 12 different pricing dates and in Figure $5.8(a)$ and $(b)$ we present the RDMs of the 43 models at 12 different pricing dates in a 3-dimension view for intuition. 


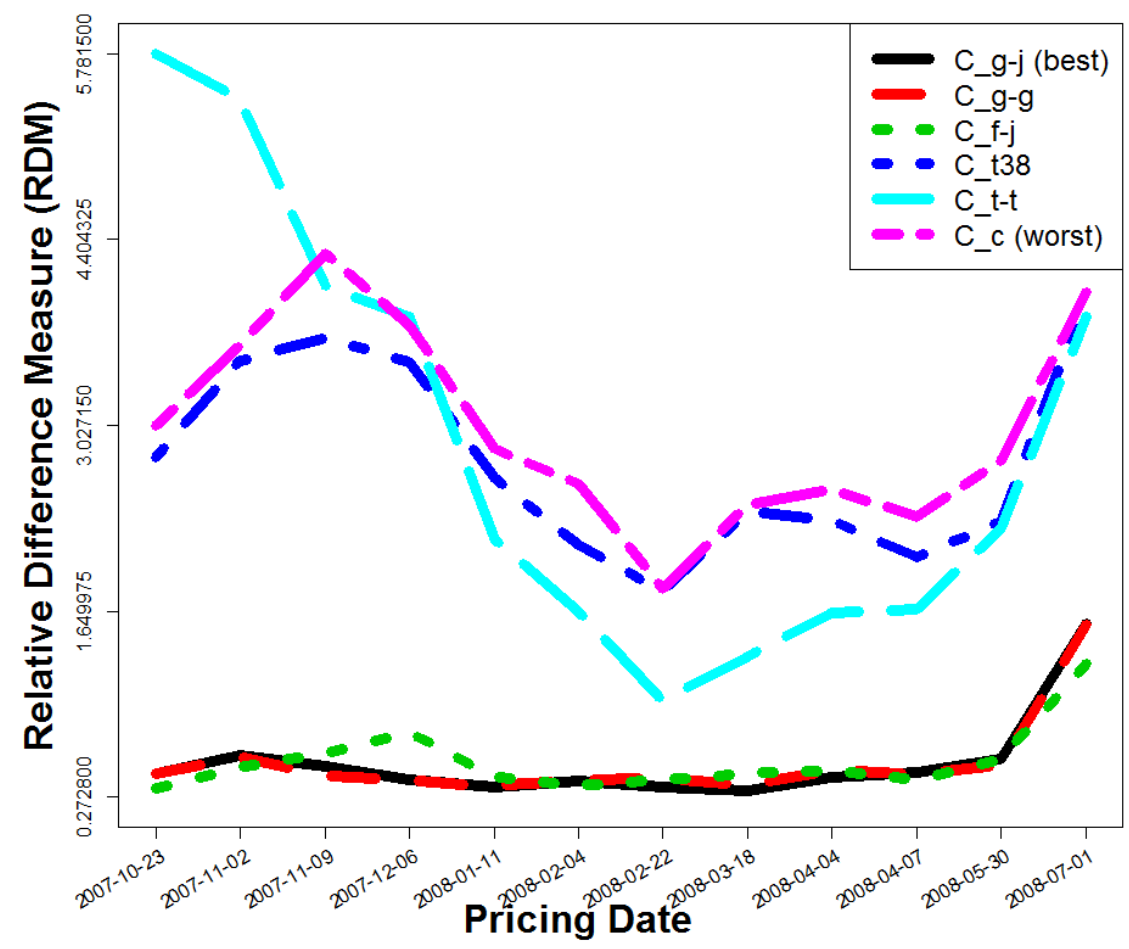

(a)

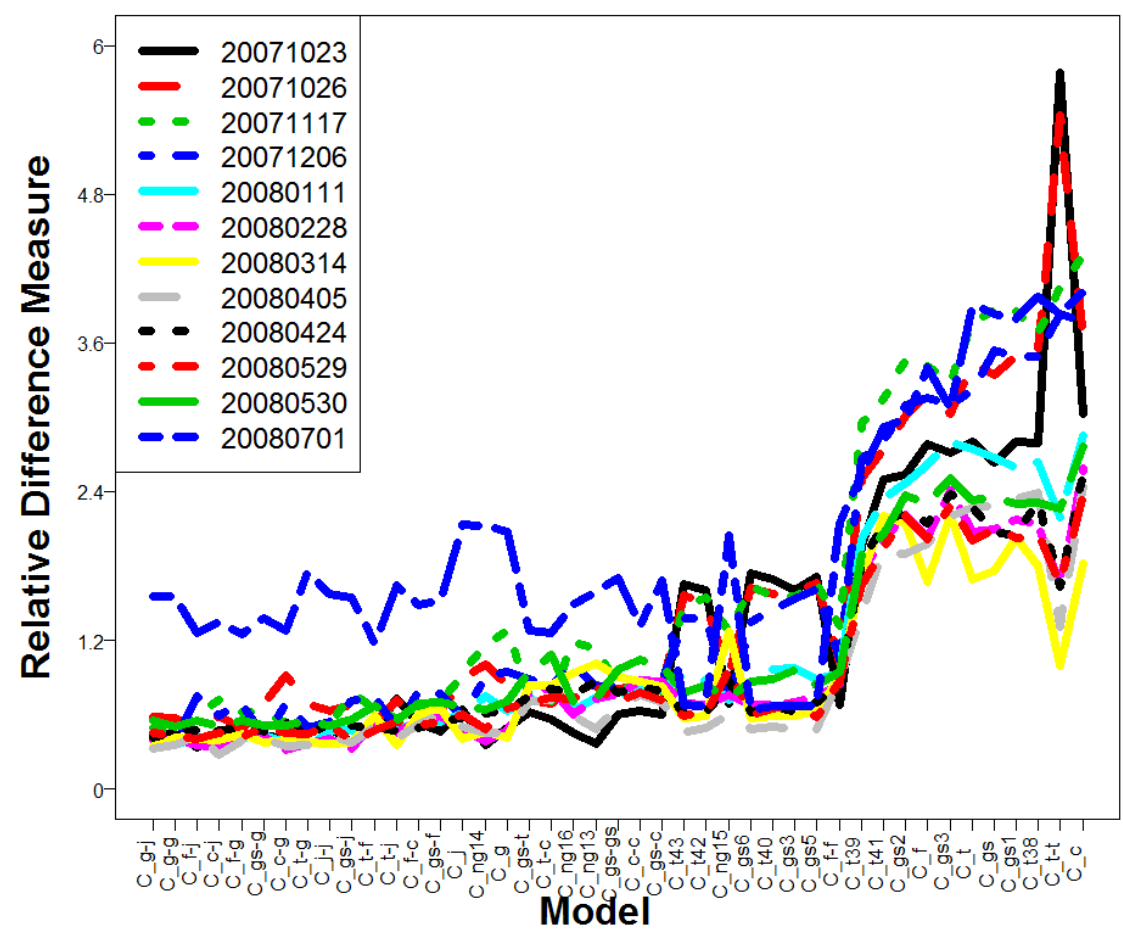

(b)

FIgURE 5.6: Comparison of models based on the relative difference measures (RDM) in Formula (5.25). (a) The best 3 models and the worst 3 models together in one graph based on the mean of 12 RDMs. (b) The RDM comparison of 43 models under 12 computation dates.

QMaRbest3worst3.R QMaRrdmcomparison.R 


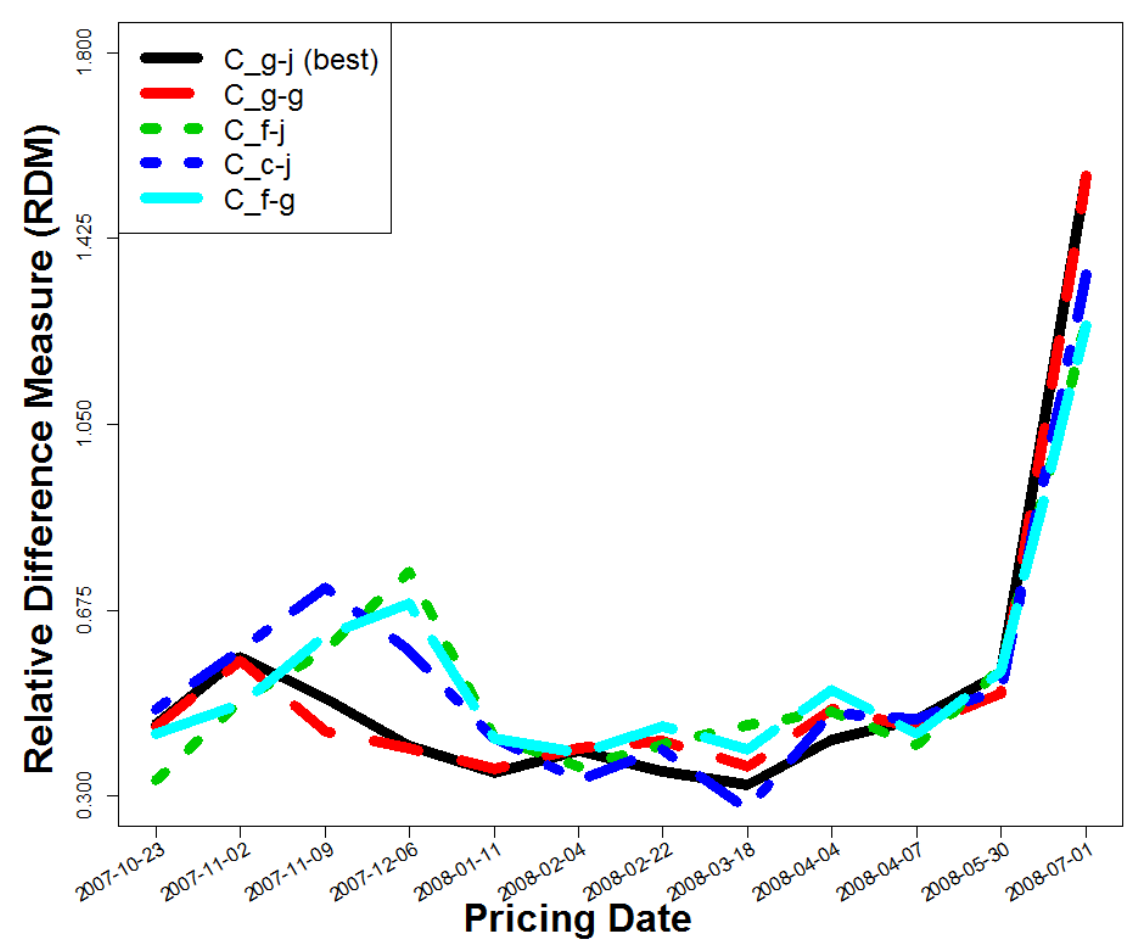

(a)

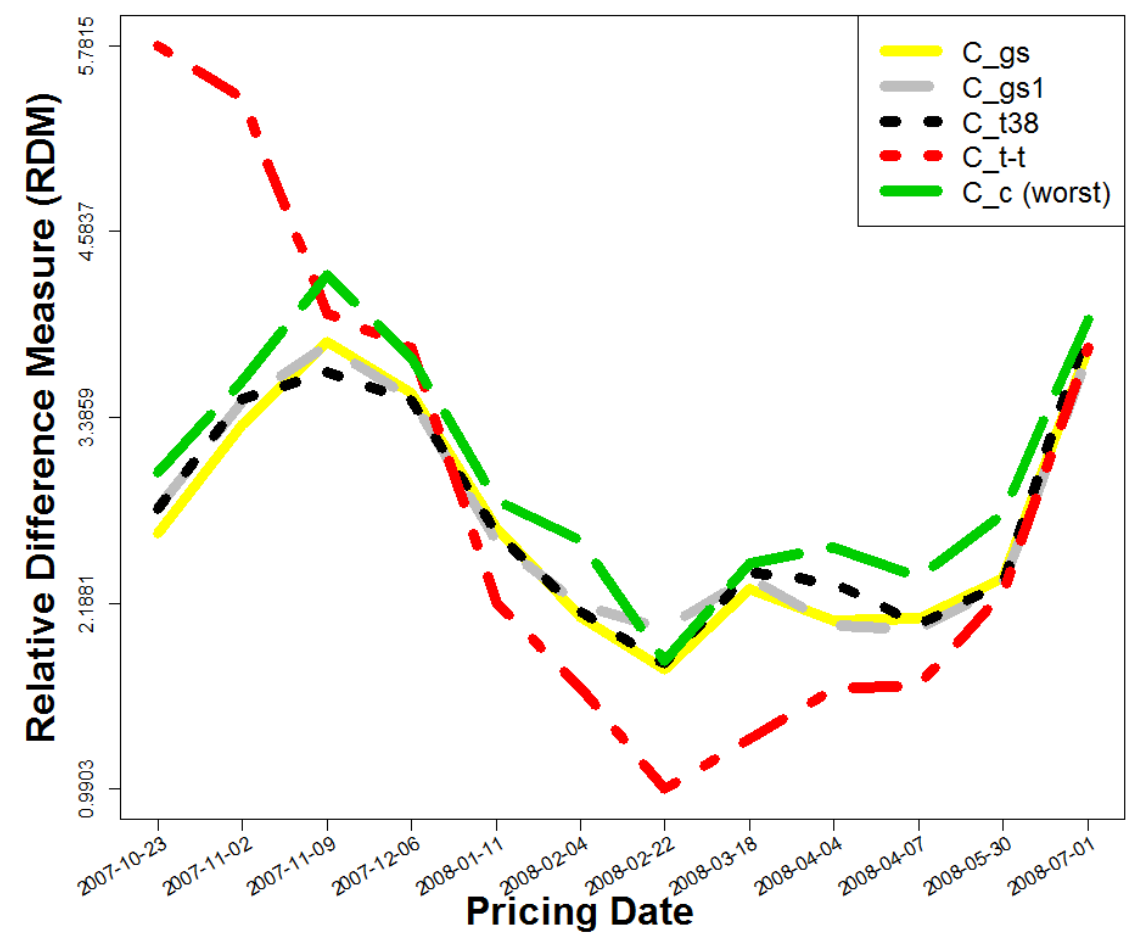

(b)

Figure 5.7: Comparison of models based on the relative difference measures (RDM) in Formula (5.25). (a) The best 5 models based on the mean of 12 RDMs. (b) The worst 5 models based on the mean of 12 RDMs.

QMaRbest5.R QMaRworst5.R 


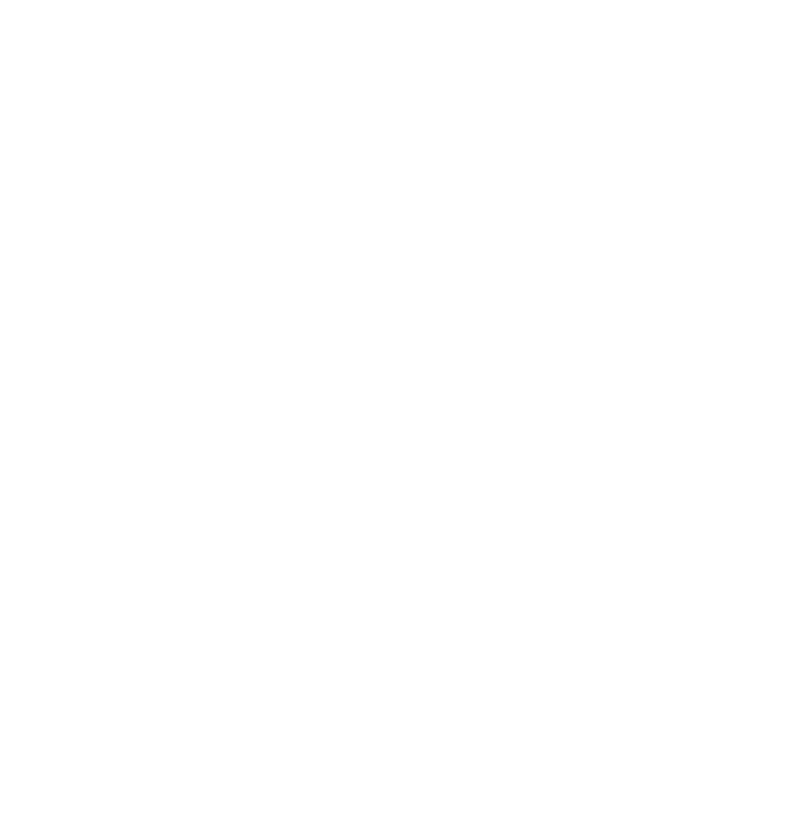

(a)

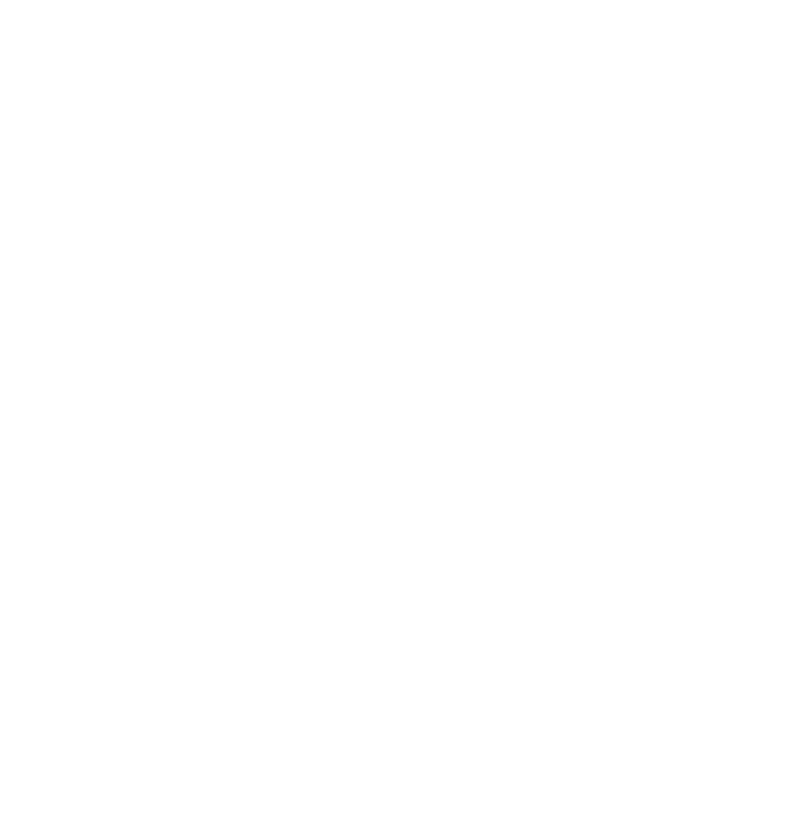

(b)

Figure 5.8: 3-dimensional illustrations of the measure RDMs of 43 models at 12 computation dates.

QmaRrdm3d.R QMaRrdm3drotate.R 


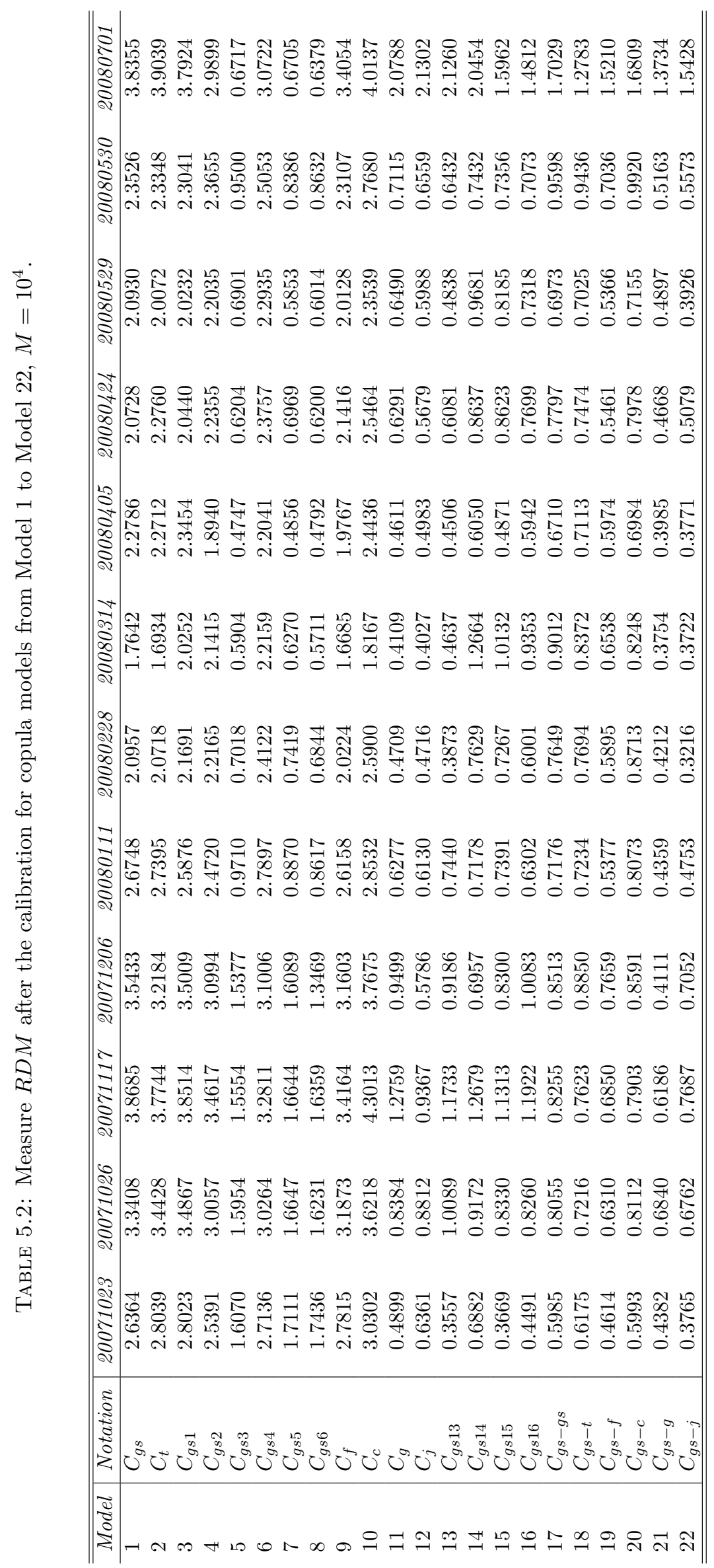




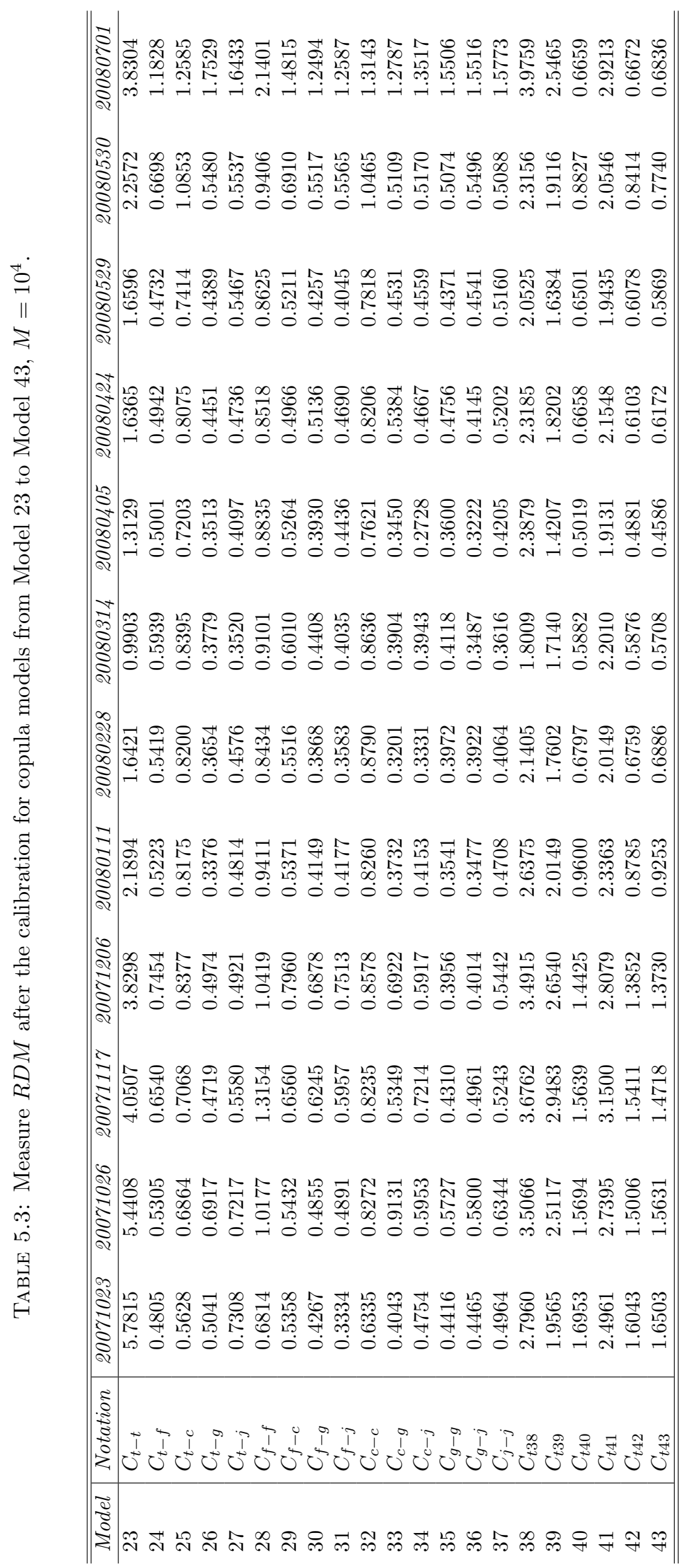




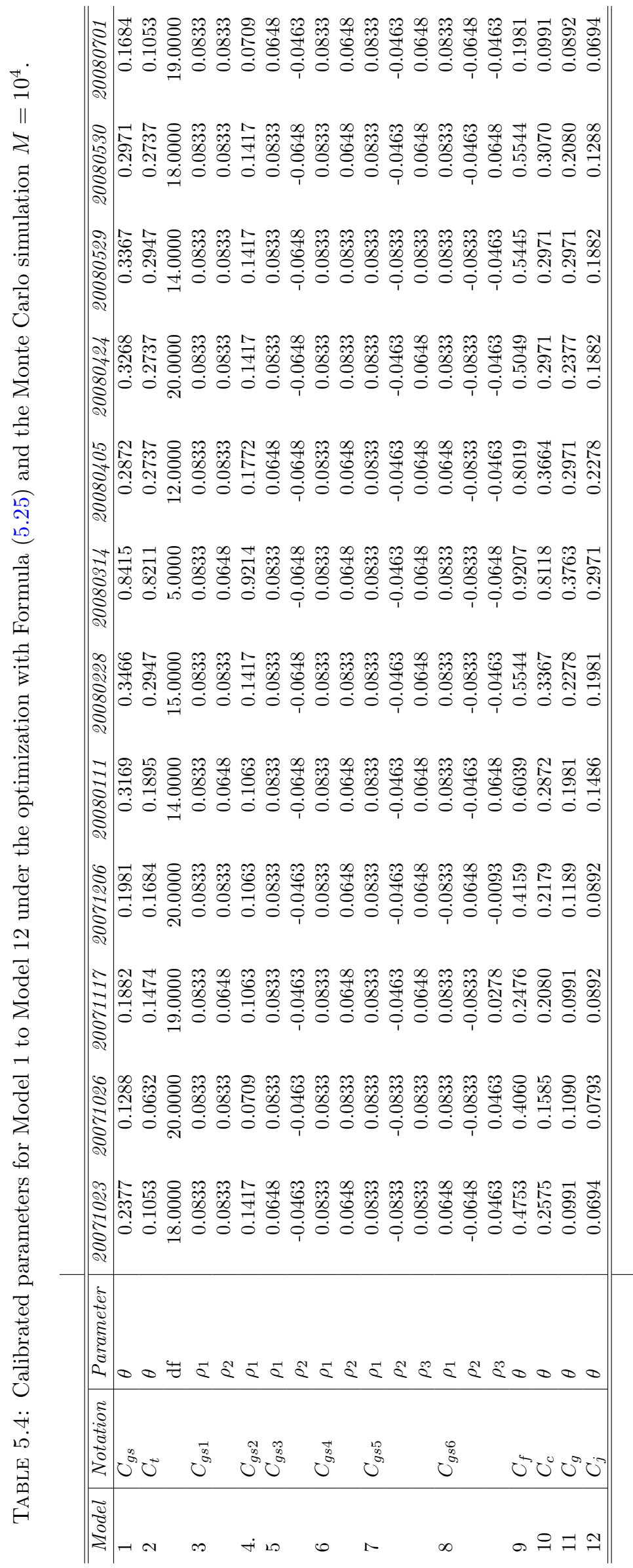




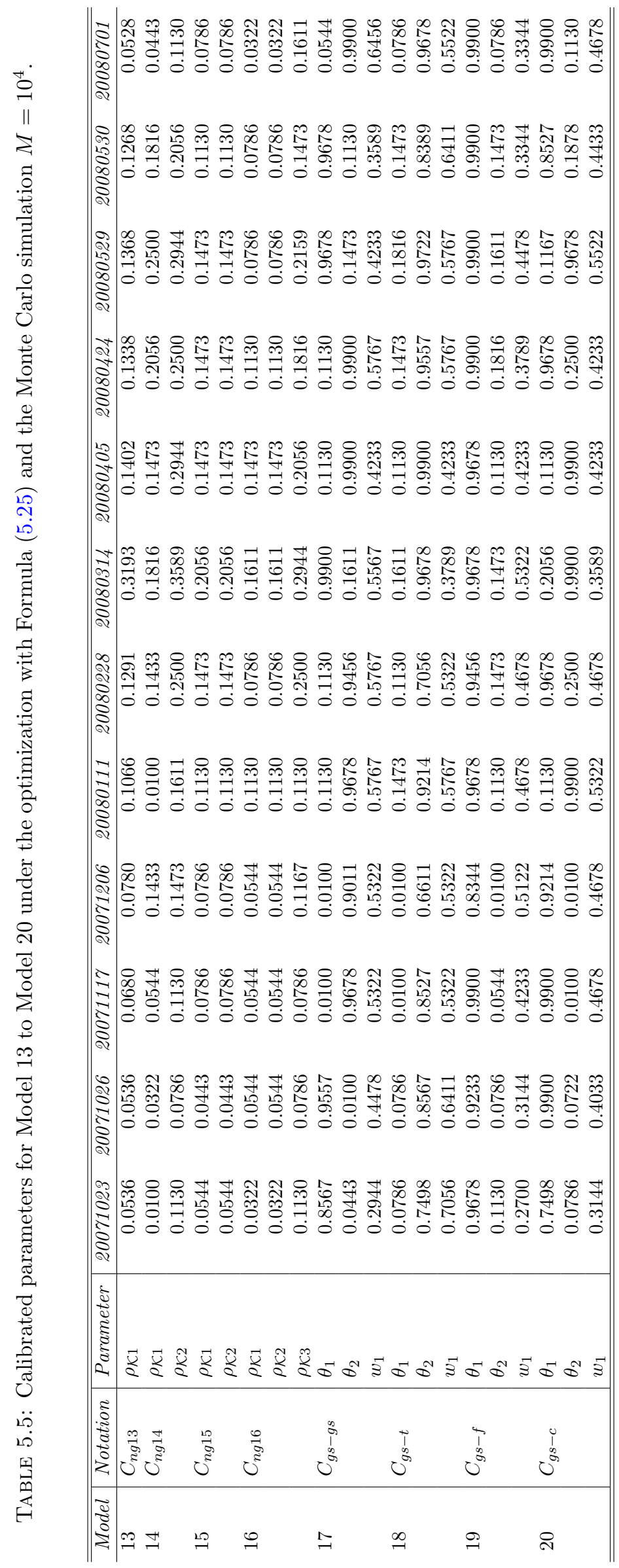




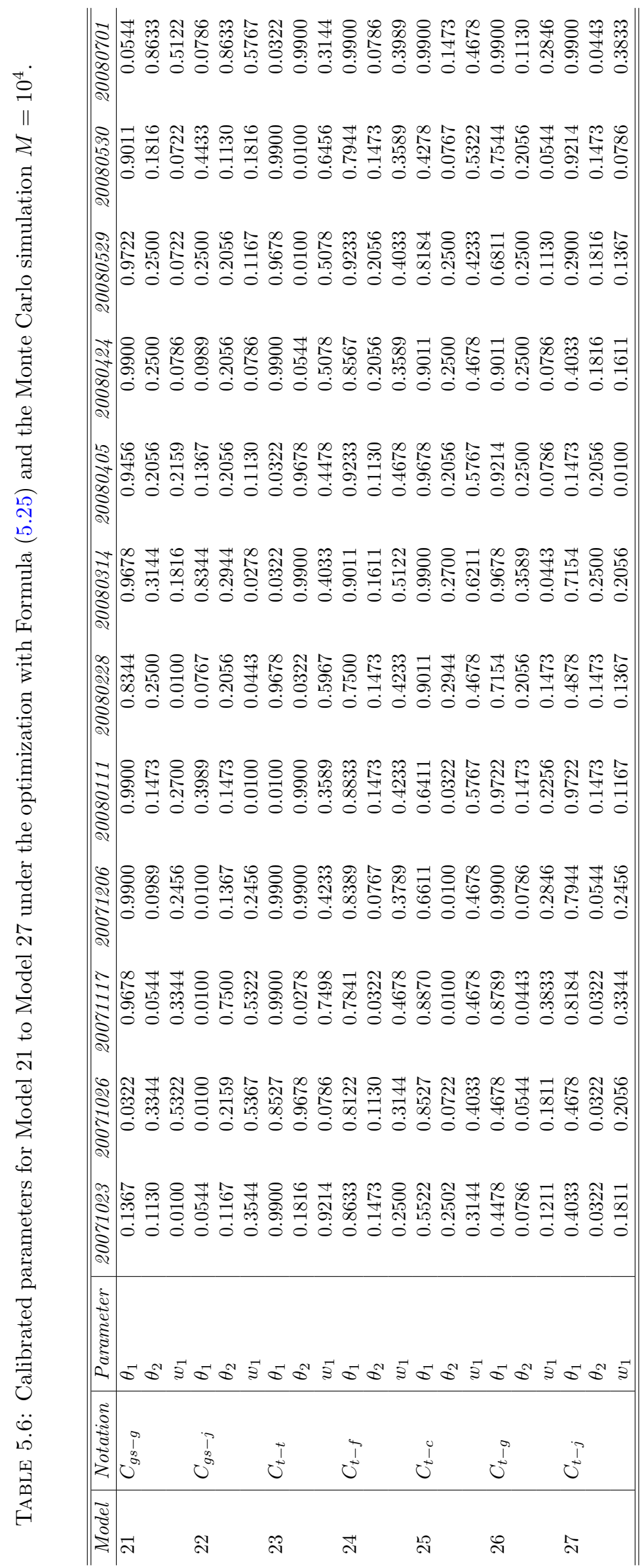




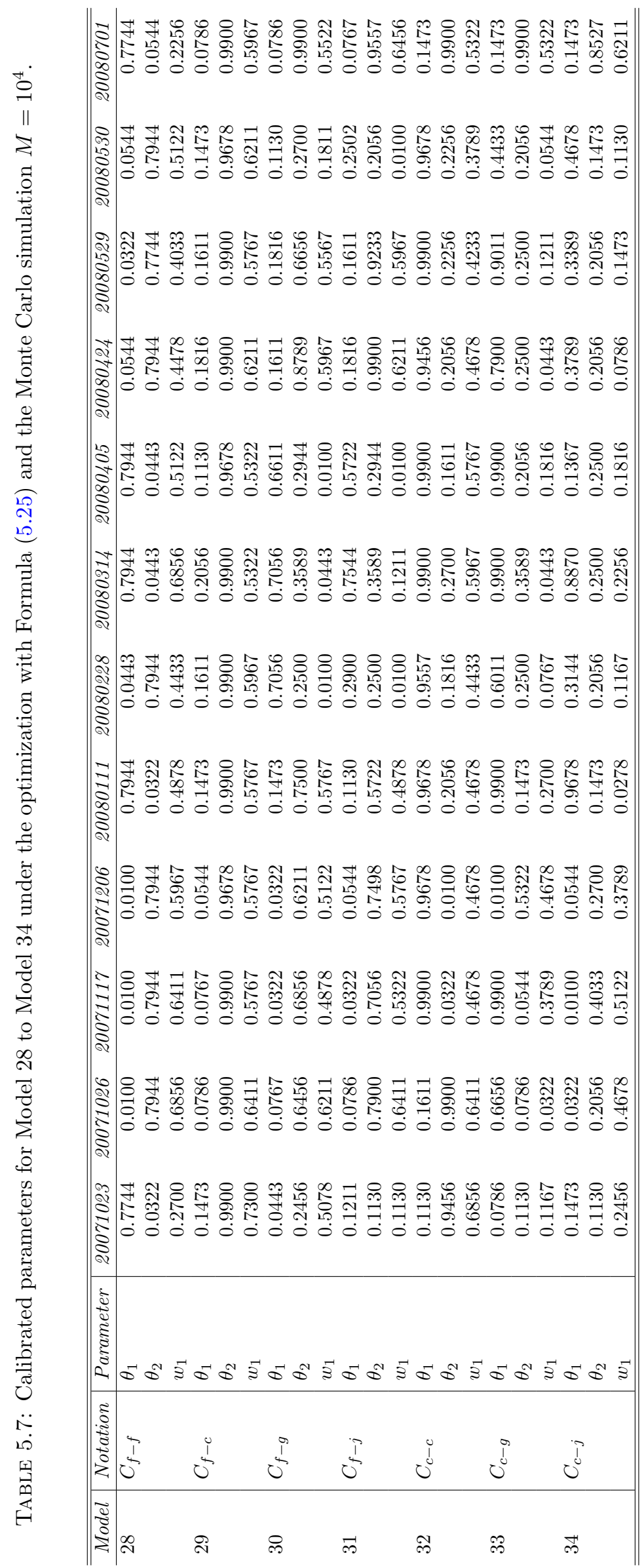




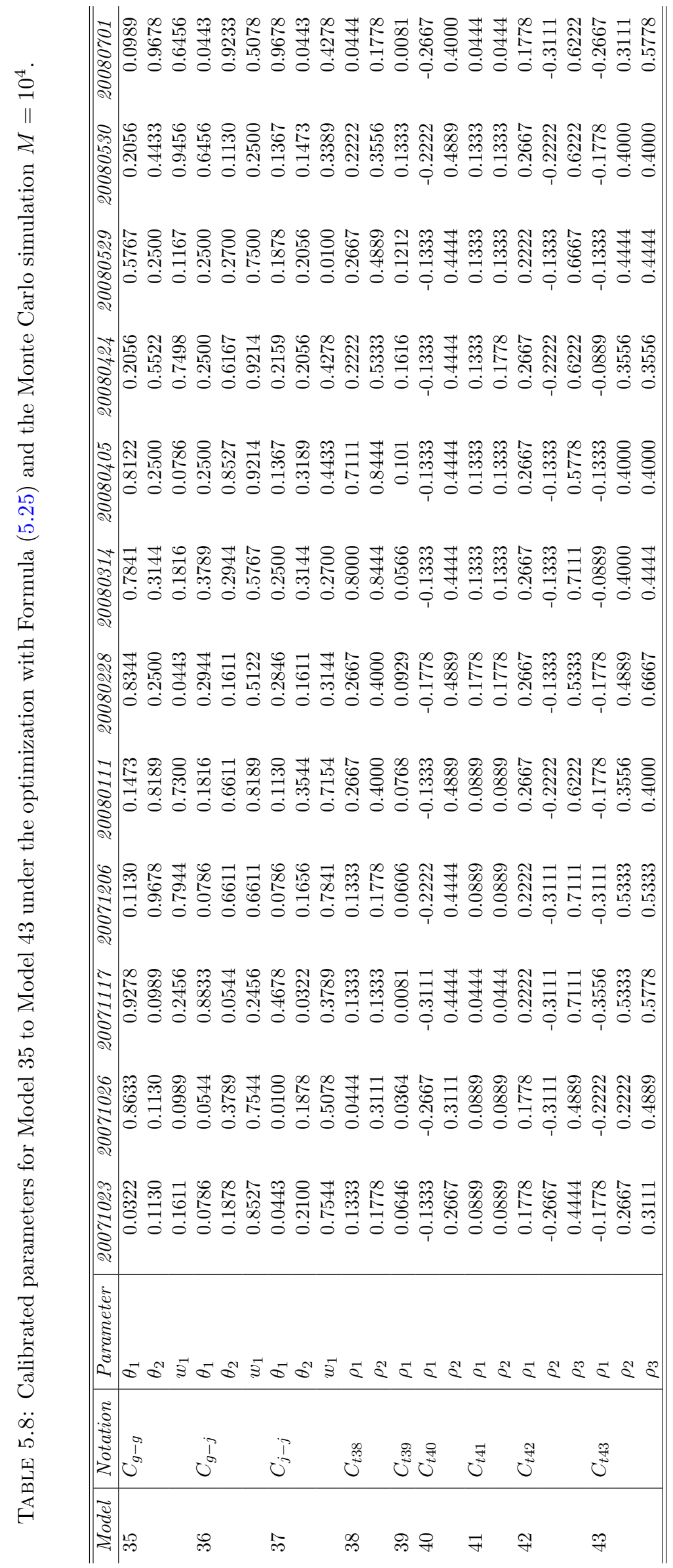




\begin{tabular}{l|l|l|l|l|l|l|l|l}
\hline \hline Rank & Notation & Mean RDM & Rank & Notation & Mean RDM & Rank & Notation & Mean RDM \\
\hline 1 & $C_{g-j}$ & 0.5254 & 16 & $C_{n g 14}$ & 0.7803 & 31 & $C_{g s 5}$ & 1.0152 \\
2 & $C_{g-g}$ & 0.5279 & 17 & $C_{g}$ & 0.7994 & 32 & $C_{f-f}$ & 1.0358 \\
3 & $C_{f-j}$ & 0.5279 & 18 & $C_{g s-t}$ & 0.8083 & 33 & $C_{t 39}$ & 2.0747 \\
4 & $C_{c-j}$ & 0.5401 & 19 & $C_{t-c}$ & 0.8236 & 34 & $C_{t 41}$ & 2.3944 \\
5 & $C_{f-g}$ & 0.5492 & 20 & $C_{n g 16}$ & 0.8271 & 35 & $C_{g s 2}$ & 2.5520 \\
6 & $C_{g s-g}$ & 0.5524 & 21 & $C_{n g 13}$ & 0.8450 & 36 & $C_{f}$ & 2.5583 \\
7 & $C_{c-g}$ & 0.5629 & 22 & $C_{g s-g s}$ & 0.8563 & 37 & $C_{g s 4}$ & 2.6659 \\
8 & $C_{t-g}$ & 0.5652 & 23 & $C_{c-c}$ & 0.8697 & 38 & $C_{t}$ & 2.7114 \\
9 & $C_{j-j}$ & 0.5817 & 24 & $C_{g s-c}$ & 0.8707 & 39 & $C_{g s}$ & 2.7130 \\
10 & $C_{g s-j}$ & 0.5894 & 25 & $C_{t 43}$ & 0.9469 & 40 & $C_{g s 1}$ & 2.7444 \\
11 & $C_{t-f}$ & 0.6157 & 26 & $C_{t 42}$ & 0.9490 & 41 & $C_{t 38}$ & 2.7583 \\
12 & $C_{t-j}$ & 0.6184 & 27 & $C_{n g 15}$ & 0.9618 & 42 & $C_{t-t}$ & 2.8851 \\
13 & $C_{f-c}$ & 0.6614 & 28 & $C_{g s 6}$ & 0.9724 & 43 & $C_{c}$ & 3.0089 \\
14 & $C_{g s-f}$ & 0.6858 & 29 & $C_{t 40}$ & 0.9888 & & & \\
15 & $C_{j}$ & 0.7476 & 30 & $C_{g s 3}$ & 0.9971 & & & \\
\hline \hline
\end{tabular}

TABLE 5.9: The ranking of 43 models under the mean RDM. Abbreviations: gs: Gaussian, $t$ : Student- $t, f$ : Frank, $c$ : Clayton, $g$ : Gumbel, $j$ : Joe, $g s i, i \in\{1, \ldots, 6\}$ : Gaussian with the correlation matrix $\rho_{g s i}, i \in\{1, \ldots, 6\}, t j, j \in\{38, \ldots, 43\}$ : Student$t$ with the same correlation matrix structure as $\rho_{g s i}, i \in\{1, \ldots, 6\}, n g$ : hierarchical Archimedean copula with the Gumbel generator function. 


\section{Chapter 6}

\section{An R Package 'CDO'}

At moment in CRAN there is no package for CDO pricing and in this master thesis we have employed 43 different copula models in CDO pricing under the context of the iTraxx Europe Index Series 8, therefore these two points motivate us in this master thesis to develop an $\mathrm{R}$ package, the package ' $\mathrm{CDO}$ '. In this chapter we give an introduction to this package and a detailed manual of the package ' $\mathrm{CDO}$ ' can be referred to https://sites.google.com/site/cdowithr/, where the package and the manual can be downloaded.

\subsection{Introduction of the Package 'CDO'}

The 'CDO' package is designed to price CDO tranches under the context of the iTraxx Europe Index Series 8. In this package 43 models has been integrated according to the following Table 6.1 which has already been shown in Section 5.1, in which Model 1 to Model 8 and Model 38 to Model 43 are elliptical copulas, Model 9 to Model 13 are single parameter Archimedean copulas, Model 14 to Model 16 are hierarchical Archimedean copulas and Model 17 to Model 37 are mixed copulas. As different CDO products may have different term structures and premium setting therefore to develop a general package for CDO pricing is difficult, therefore we set our computation focusing only on the 12 pricing dates, 20071023, 20071102, 20071109, 20071206, 20080111, 20080204, 20080222, 20080318, 20080407, 20080530, 20080701, in iTraxx Europe Index Series 8, which is already studied in Hofert and Scherer [8], Choros-Tomczyk et al. [38], Hofert 


\begin{tabular}{l|l|l|l|l|l}
\hline \hline Model & Notation & Model & Notation & Model & Notation \\
\hline 1 & $C_{g s}$ & 14 & $C_{n g 14}$ & 27 & $C_{t-j}$ \\
2 & $C_{t}$ & 15 & $C_{n g 15}$ & 28 & $C_{f-f}$ \\
3 & $C_{g s 1}$ & 16 & $C_{n g 16}$ & 29 & $C_{f-c}$ \\
4 & $C_{g s 2}$ & 17 & $C_{g s-g s}$ & 30 & $C_{f-g}$ \\
5 & $C_{g s 3}$ & 18 & $C_{g s-t}$ & 31 & $C_{f-j}$ \\
6 & $C_{g s 4}$ & 19 & $C_{g s-f}$ & 32 & $C_{c-c}$ \\
7 & $C_{g s 5}$ & 20 & $C_{g s-c}$ & 33 & $C_{c-g}$ \\
8 & $C_{g s 6}$ & 21 & $C_{g s-g}$ & 34 & $C_{c-j}$ \\
9 & $C_{f}$ & 22 & $C_{g s-j}$ & 35 & $C_{g-g}$ \\
10 & $C_{c}$ & 23 & $C_{t-t}$ & 36 & $C_{g-j}$ \\
11 & $C_{g}$ & 24 & $C_{t-f}$ & 37 & $C_{j-j}$ \\
12 & $C_{j}$ & 25 & $C_{t-c}$ & & \\
13 & $C_{n g 13}$ & 26 & $C_{t-g}$ & & \\
\hline \hline
\end{tabular}

TABle 6.1: List of employed copula models. Abbreviations: $g s$ : Gaussian, $t$ : Student$t, f$ : Frank, $c$ : Clayton, $g$ : Gumbel, $j$ : Joe, gsi, $i \in\{1, \ldots, 6\}$ : Gaussian with the correlation matrix $\rho_{g s i}, i \in\{1, \ldots, 6\}, t j, j \in\{38, \ldots, 43\}$ : Student- $t$ using the correlation matrix structure $\rho_{g s i}, i \in\{1, \ldots, 6\}, n g$ : hierarchical Archimedean copula with the Gumbel generator function.

[9], Choros-Tomczyk et al. [10], Choros-Tomczyk et al. [39] and Choros-Tomczyk et al. $[40]$.

\subsection{Installation and Usage Examples}

Firstly, user needs to note that the package 'CDO' is constructed under the R(3.0.3), therefore user needs to update the $\mathrm{R}$ version equal or higher than this version, i.e. $\mathrm{R}>=$ 3.0.3. Then user needs to download two data sets, payDay.csv for setting the pricing dates and Def Intensity.csv for setting the default intensity consistent with the pricing date. Both data sets can be obtained from the repository xyfQuantlet in Github.

Secondly, user needs to install the package 'CDO', which can be done with 2 methods. The first method is very easy, which is an automatic installation by 4 lines $\mathrm{R}$ codes given as follows,

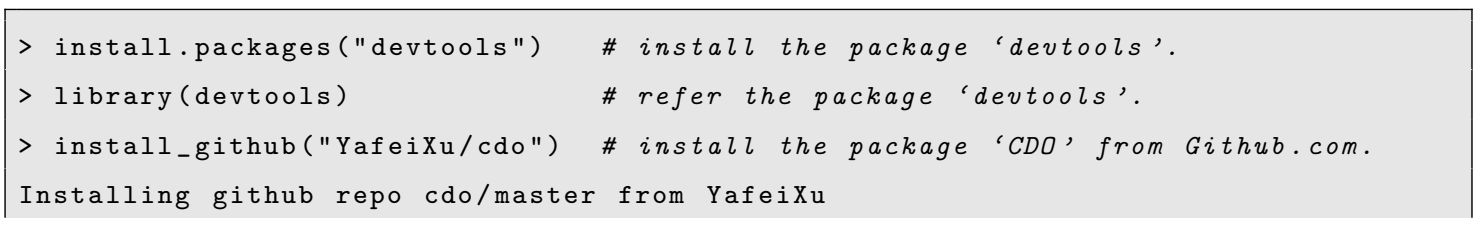




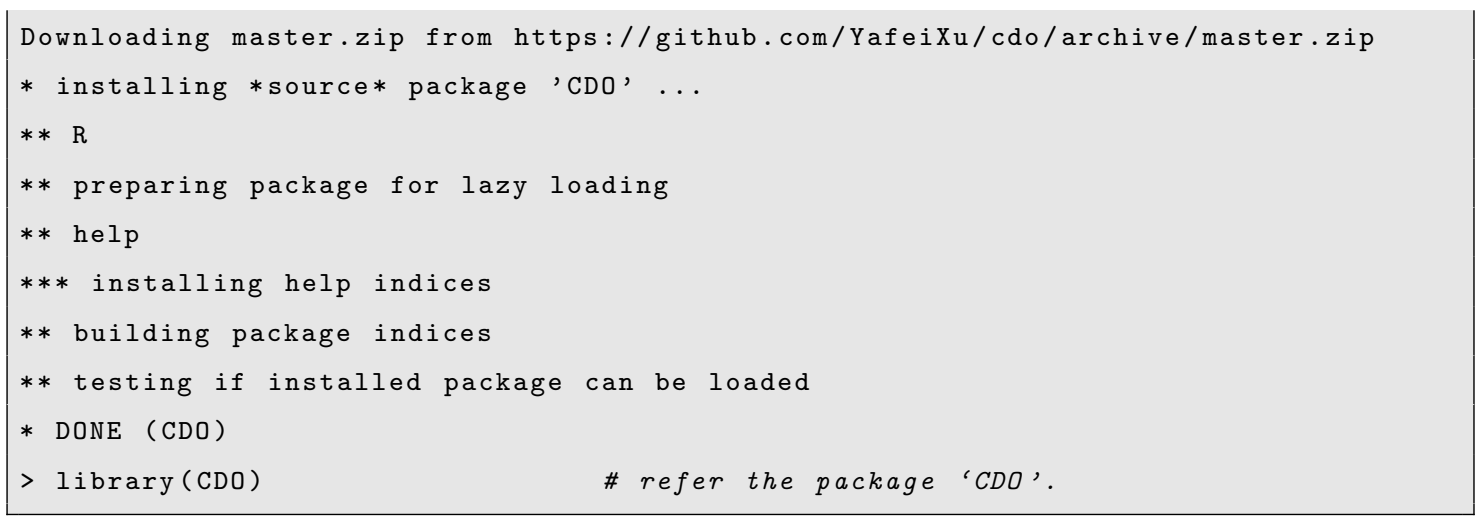

In the second method for installation user needs to download the package 'CDO' with the aforementioned LINK, and then install the package manually under the Rgui interface. The package is constructed based on the R 3.0.3 version and is dependent on the package 'copula' referred to Yan [41] and Marius Hofert and Yan [42] and the package 'matrixcalc' referred to Novomestky [43]. After installation then we can use 'CDO' to price tranches spreads. Following we give three examples using the 'CDO' including the David Li's Gaussian copula model, Model 1, the hierarchical Archimedean copula model with the Gumbel generator function, Model 16, and the mixed Gumbel-Joe copula model, Model 36 .

\section{Example 1}

Price the 5 CDS index tranches on 20071023 with 10000 runs of Monte Carlo simulation employing the David X. Li's Gaussian copula model in Li [1] and Li [2] such that,

$$
C\left(u_{1}, \ldots, u_{K} ; \theta\right)=C_{g s}\left(u_{1}, \ldots, u_{K} ; \theta\right)
$$

The $\mathrm{R}$ codes can be given as follows,

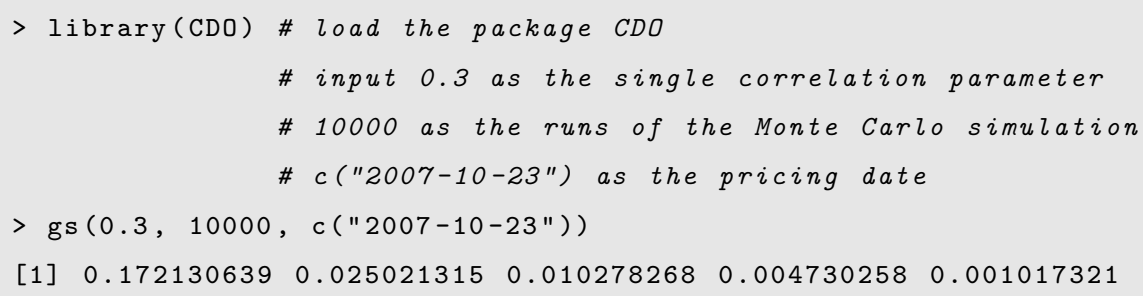

Therefore we can observe the results that the upfront payment rate for the equity tranche is 0.1721 , the other four tranche spreads are correspondingly 0.0250, 0.0102, 0.0047, 0.0010 . 


\section{Example 2}

Price the 5 CDS index tranches on 20080530 with 10000 runs of Monte Carlo simulation employing the hierarchical Archimedean copula with a Gumbel copula function in Choros-Tomczyk et al. [10], Model $16 C_{n g 16}$ in Table 6.1, such that

$$
\begin{aligned}
C\left(u_{1}, \ldots, u_{K}, u_{K+1}, \ldots, u_{K+6} ; \theta\right)= & C_{n g 16}^{1}\{ \\
& C_{n g 16}^{2}\left[u_{K+1}, C_{n g 16}^{3}\left(u_{1}, \ldots, u_{s_{1}} ; \rho_{\mathcal{K} 3}\right) ; \rho_{\mathcal{K} 2}\right], \\
& C_{n g 16}^{2}\left[u_{K+2}, C_{n g 16}^{3}\left(u_{s_{1}+1}, \ldots, u_{s_{1}+s_{2}} ; \rho_{\mathcal{K} 3}\right) ; \rho_{\mathcal{K} 2}\right], \ldots, \\
& C_{n g 16}^{2}\left[u_{K+6}, C_{n g 16}^{3}\left(u_{s_{1}+\cdots+s_{5}+1}, \ldots, u_{K} ; \rho_{\mathcal{K} 3}\right) ; \rho_{\mathcal{K} 2}\right] ; \rho_{\mathcal{K} 1} \\
& \} .
\end{aligned}
$$

The $R$ codes can be given as follows,

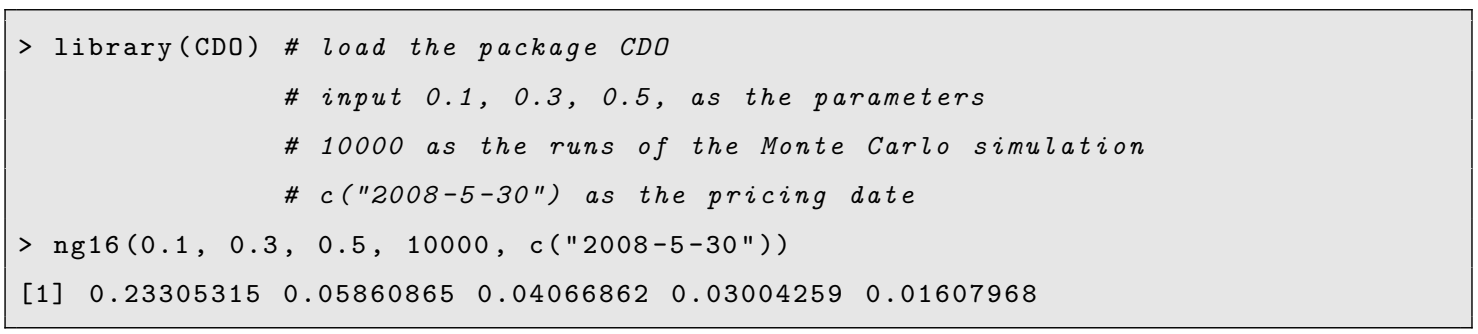

Therefore we can observe the results that the upfront payment rate for the equity tranche is 0.2330 , the other four tranche spreads are correspondingly 0.0586, 0.0406, 0.0300, 0.0160 .

\section{Example 3}

Price the 5 CDS index tranches on 20080404 with 10000 runs of Monte Carlo simulation employing the mixed Gumbel-Joe copula $C_{g-j}$, which perform the best in the RDM ranking, such that,

$$
C_{g-j}\left(u_{1}, \ldots, u_{K} ; \theta\right)=w_{1} C_{g}\left(u_{1}, \ldots, u_{K} ; \theta_{1}\right)+w_{2} C_{j}\left(u_{1}, \ldots, u_{K} ; \theta_{2}\right)
$$

The $R$ codes can be given as follows,

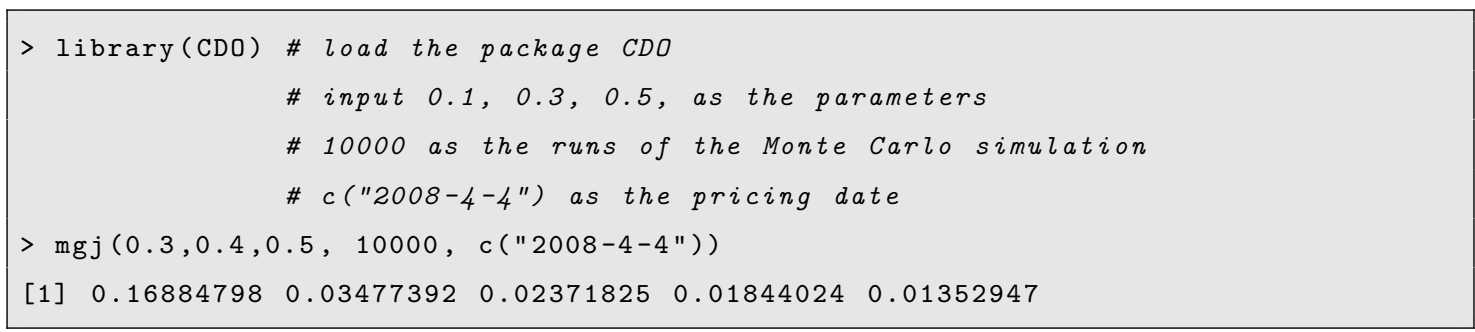


Therefore we can observe the results that the upfront payment rate for the equity tranche is 0.1688 , the other four tranche spreads are correspondingly 0.0347, 0.0237, 0.0184, 0.0135 . 


\section{Chapter 7}

\section{Conclusion}

The goal of this master thesis is to construct defaults dependence with copulas for the CDS index tranche pricing. As $\mathrm{Li}$ [1] and $\mathrm{Li}$ [2] have introduced the copula approach into CDO pricing with an exchangeable Gaussian copula, which has become an industry standard, albeit the exchangeable Gaussian copula does not possess features such as the heterogeneous dependence between sectors, the non-elliptical distribution and the asymmetrical tail-dependence, which are exposed in the joint behavior of multi-name defaults, therefore several studies have contributed in exploring new copulas in order to improve the pricing of CDOs, for which the elliptical copulas, the Archimedean copulas and the hierarchical Archimedean copulas have been investigated.

Our master thesis focuses on the utilization of the mixed copula, a linear combination of copulas, which has been studied in $\mathrm{Hu}$ [34], Wang [36] and Cai et al. [37], where the joint collapses of stock markets have been researched, which shows good performance of the mixed copula in modeling joint behaviors in financial markets. We employ in this thesis the mixed copula to model the joint defaults, which is an important step in the CDS index tranche pricing.

In this master thesis we totally applied 43 diverse copula models containing 14 elliptical copulas, 5 single parameter Archimedean copulas, 3 hierarchical Archimedean copulas and 21 mixed copulas with two component copulas coming from 2 elliptical copulas and 4 Archimedean copulas. For all the 43 models employed in this master thesis we refer to the Table 6.1. At last we give out all computation results based on the relative difference measure $(\mathrm{RDM})$. We find that the mixed copulas have superior performance than 
other copula models. Especially those mixed copulas which own at least one component copula coming from the Gumbel copula or the Joe copula, show a top performance, which is a clear evidence that joint defaults are right tail-dependent. And in the other three families, i.e. the elliptical family, the Archimedean family and the hierarchical Archimedean family, the elliptical family performs the worst, which means that those copulas without the asymmetrical tail-dependence feature is not suitable for the CDS index tranche pricing, and the rest two families, the Archimedean copula and the hierarchical Archimedean copula perform very similar. For the ranking of all 43 models based on the mean of RDMs we refer to the Table 5.9. As there is not an $\mathrm{R}$ package by now for the CDO tranche pricing, therefore we have developed the R package 'CDO', which can be used to price the CDS index tranches in iTraxx Index Series 8. And all the empirical results in this master thesis can also be reproduced by this package. 


\section{Bibliography}

[1] David X. Li. The valuation of basket credit derivatives. CreditMetrics Monitor, pages 34-50, 1999.

[2] David X. Li. On default correlation: A copula function approach. Journal of Fixed Income, 9:43-54, 2000.

[3] Leif Andersen. Lectures notes for interest rate and credit modeling. Lectures notes, 2006.

[4] Oldrich Alfons Vasicek. Limiting loan loss probability distribution. KMV Coporation Technical Report, 1991.

[5] Oldrich Alfons Vasicek. The distribution of loan portfolio value, risk. KMV Coporation Technical Report, 2002.

[6] Robert Merton. On the pricing of corporate debt: the risk structure of interest rates. Journal of Finance, 2:449C470, 1974.

[7] Ruediger Frey and Alexander J. McNeil. Dependent defaults in models of portfolio credit risk. Journal of Risk, 6:56-92, 2003.

[8] Marius Hofert and Matthias Scherer. Cdo pricing with nested archimedean copulas. Working Paper, 2008.

[9] Jan Marius Hofert. Sampling nested archimedean copulas with applications to cdo pricing. Dissertation at the Ulm University, 2010.

[10] Barbara Choros-Tomczyk, Wolfgang Karl Haerdle, and Ostap Okhrin. Valuation of collateralized debt obligations with hierarchical archimedean copulae. Journal of Empirical Finance, 24:42-62, 2013. 
[11] Filip Lindskog and Alexander McNeil. Common poisson shock models: Applications to insurance and credit risk modelling. ASTIN BULLETIN, 33:209-238, 2001.

[12] Paul Embrechts, Filip Lindskog, and Alexander McNeil. Modelling dependence with copulas and applications to risk management. Working Paper, 2001.

[13] Leif Andersen, Jakob Sidenius, and Susanta Basu. Credit derivatives: All your hedges in one basket. Risk, 16:67-72, 2003.

[14] Andrei Greenberg, Roy Mashal, Marco Naldi, and Lutz Schloegl. Tuning correlation and tail risk to the market prices of liquid tranches. Lehman Brothers- Quantitative Credit Research, 2004.

[15] Roy Mashal, Marco Naldi, and Assaf Zeevi. Comparing the dependence structure of equity and asset returns. Credit risk models and management - London - Risk Books, pages 403-417, 2004.

[16] Stefano Demarta and Alexander J. McNeil. The t copula and related copulas. International Statistical Review, 73:111-129, 2005.

[17] Lutz Schloegl and Dominic O'Kane. A note on the large homogeneous portfolio approximation with the student-t copula. Finance Stochastics, 9:577-584, 2005.

[18] Philipp Schoenbucher and Dirk Schubert. Copula-dependent default risk in intensity models. Working Paper, 2000.

[19] Philipp Schoenbucher. Taken to the limit: Simple and not-so-simple loan loss distribution. Working Paper, 2002.

[20] Ebbe Rogge and Phillip Schoenbucher. Modelling dynamic portfolio credit risk. Working Paper, 2003.

[21] J. Gregory and J.-P. Laurent. Basket default swaps, cdo's and factor copulas. Risk, $7,2003$.

[22] Jon Gregory and Jean-Paul Laurent. I will survive. Risk, 6:103-107, 2003.

[23] Andrew Friend and Ebbe Rogge. Correlation at first sight. Working Paper, 2004.

[24] Darrell Duffie and Kenneth Singleton. Simulating correlated defaults. Working Paper, 1998. 
[25] Kay Giesecke. A simple exponential model for dependent defaults. Journal of Fixed Income, 13:74-83, 2003.

[26] John Hull and Alan White. Valuation of a cdo and an nth to default cds without monte carlo simulation. Journal of Derivatives, 12:8-23, 2004.

[27] Mary Childs. Synthetic cdos making comeback as yields juiced. Bloomberg, 2013.

[28] Markit. Markit itraxx europe indices series 20. Technical Report, 2013.

[29] Wassilij Hoeffding. Masstabinvariante korrelationstheorie. Schriften des Matematischen Instituts und des Instituts fuer Angewandte Matematik der Universitat Berlin, 5:179-233, 1940.

[30] Abe Sklar. Fonctions de repartition a n dimensions et leurs marges. Publications de l'Institut de statistique de l'Universit de Paris, 8:299-231, 1959.

[31] Harry Joe. Multivariate Models and Dependence Concepts. Chapman \& HallLondon, 1997.

[32] Roger Nelsen. An Introduction to Copulas. Springer- New York, 2006.

[33] Eric Bouye. Copulas for finance: A reading guide and some applications. Working Paper, 2000.

[34] Ling Hu. Dependence patterns across financial markets: A mixed copula approach. Applied Financial Economics, 16:717-729, 2006.

[35] Ostap Okhrin, Yarema Okhrin, and Wolfgang Schmid. On the structure and estimation of hierarchical archimedean copulas. Journal of Econometrics, 173(2): 189-204, 2013.

[36] Xian Wang. Selection of mixed copulas and finite mixture models with applications in finance. Dissertation at the University of North Carolina at Charlotte, 2008.

[37] Zongwu Cai, Xiaohong Chen, Yanqin Fan, and Xian Wang. Selection of copulas for risk management. Working Paper, 2009.

[38] Barbara Choros-Tomczyk, Wolfgang Karl Haerdle, and Ostap Okhrin. Cdo pricing with copulae. Bulletin of the International Statistical Institute, 57th Session, 57, 2009. 
[39] Barbara Choros-Tomczyk, Wofgang Haerdle, and Ludger Overbeck. Copula dynamics in cdos. Quantitative Finance, 2014.

[40] Barbara Choros-Tomczyk, Wolfgang Haerdle, and Ostap Okhrin. Cdo surfaces dynamics. Discussion paper, SFB 649, Humboldt Universitaet zu Berlin, 2013.

[41] Jun Yan. Enjoy the joy of copulas: With a package copula. Journal of Statistical Software, 21(4), 2007.

[42] Martin Maechler Marius Hofert, Ivan Kojadinovic and Jun Yan. Package 'copula'. R Package Manual, 2014.

[43] Frederick Novomestky. Package 'matrixcalc'. R Package Manual, 2013. 


\section{Declaration of Authorship}

I hereby confirm that I have authored this Master's thesis independently and without use of others than the indicated sources. All passages which are literally or in general matter taken out of publications or other sources are marked as such.

Berlin, March 31, 2014

Yafei Xu 


\section{Package 'CDO'}

May 29, 2014

Type Package

Title This package is designed to reproduce the iTraxx Europe Index Series 8 tranche spreads.

Version 1.3

Date 2014-04-07

Author Yafei Xu<yafei.xu@hu-berlin.de>

Maintainer Yafei Xu <yafei.xu@hu-berlin.de>

Depends copula, matrixcalc, $\mathrm{R}(>=3.0 .3)$

\section{Description}

In this package 43 models, containing elliptical copula models, Archimedean copula models, hierarchical Archimedean copula models and mixed copula models, have been employed for pricing the iTraxx Europe Index Series 8 tranche spreads. Details for 43 models and the computations can be referred to the master theisis, CDO, HAME Copulas and an R Package 'CDO'. IMPORTANT: This package requires the $\mathrm{R}>=3.0 .3$. And please download the data sets of "defIntensity.csv" and "payday.csv" from "https://github.com/YafeiXu/xyfQuantlet" and correctly install them in such paths: “C:/defIntensity.csv", "C:/payday.csv".

URL https://sites.google.com/site/cdowithr

NeedsCompilation no

Repository https://github.com/YafeiXu/CDO

Date/Publication 2014-04-07

License GPL (>=3)

\section{R topics documented:}

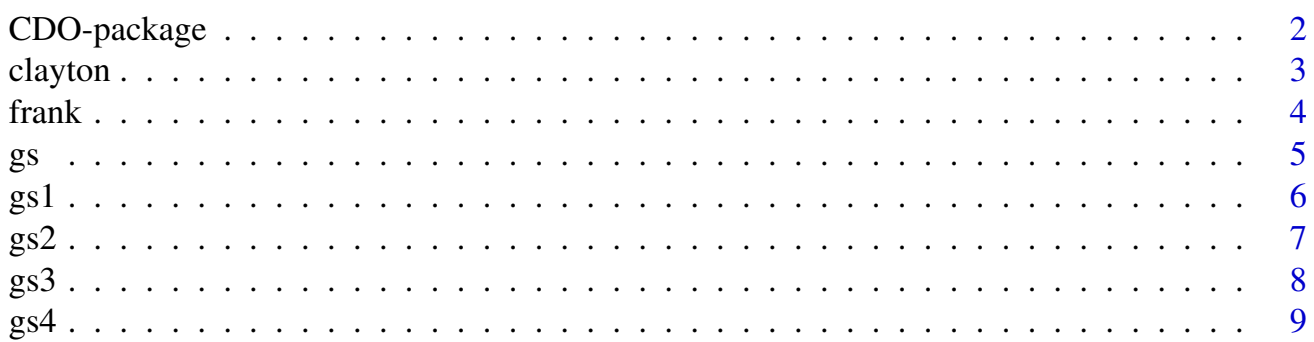




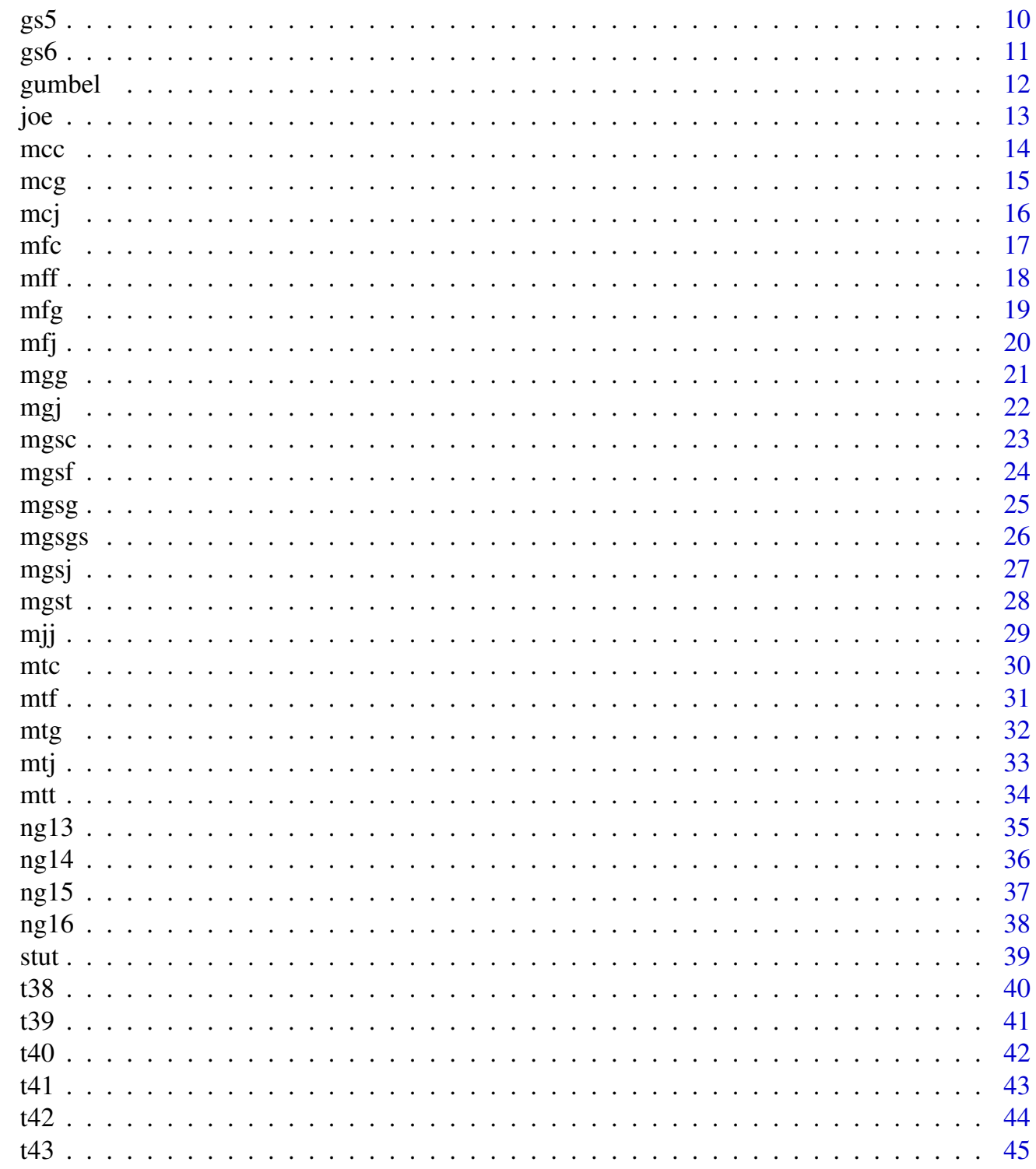

Index

\section{Description}

This package includes 43 models, introduced in the master thesis CDO, HAME Copulas and an R Package 'CDO', for CDS index tranche pricing under the context of iTraxx Europe Index Series 8. 


\section{Details}

$\begin{array}{ll}\text { Package: } & \text { CDO } \\ \text { Type: } & \text { Package } \\ \text { Version: } & 1.3 \\ \text { Date: } & 2014-04-07 \\ \text { License: } & \text { GPL }(>=3)\end{array}$

All 43 models can be found in the master thesis CDO, HAME Copulas and an R Package 'CDO' by Yafei Xu at Humboldt-Universitaet zu Berlin.

\section{Author(s)}

Yafei Xu

Maintainer: Yafei Xu <yafei.xu@hu-berlin.de>

\section{References}

<https://sites.google.com/site/cdowithr/>

\section{Description}

'clayton' computes 5 tranches' spreads under the Clayton copula.

\section{Usage}

clayton(rhoInput, MInput, dateInput)

\section{Arguments}

rhoInput a numeric giving the Kendeall's tau for dependence specification.

MInput a numeric giving the Monte Carlo simulation runs.

dateInput a character giving the pricing date, e.g. c("2007-10-23").

\section{Details}

Please make sure that the data sets of "defIntensity.csv" and "payday.csv" have been correctly installed in such paths: "C:/defIntensity.csv", "C:/payday.csv". The both data sets can be downloaded from "https://github.com/YafeiXu/xyfQuantlet".

\section{Value}

A vector with 5 numerics will be returned, from left to right: equity, junior mezzanine, senior mezzanine, junior senior, senior. 


\section{Author(s)}

Yafei Xu<yafei.xu@hu-berlin.de>

\section{References}

The master thesis, CDO, HAME Copulas and an R Package 'CDO', can be downloaded from https://sites.google.com/site/cdowithr/.

\section{Examples}

clayton( $0.3,10000, c(" 2007-10-23 "))$

frank CDO Pricing with the Frank Copula

\section{Description}

'frank' computes 5 tranches spreads under the Frank copula.

\section{Usage}

frank(rhoInput, MInput, dateInput)

\section{Arguments}

rhoInput a numeric giving the Kendeall's tau for dependence specification.

MInput a numeric giving the Monte Carlo simulation runs.

dateInput a character giving the pricing date, e.g. c("2007-10-23").

\section{Details}

Please make sure that the data sets of "defIntensity.csv" and "payday.csv" have been correctly installed in such paths: "C:/defIntensity.csv", "C:/payday.csv". The both data sets can be downloaded from "https://github.com/YafeiXu/xyfQuantlet".

\section{Value}

A vector with 5 numerics will be returned, from left to right: equity, junior mezzanine, senior mezzanine, junior senior, senior.

\section{Author(s)}

Yafei Xu <yafei.xu@hu-berlin.de>

\section{References}

The master thesis, CDO, HAME Copulas and an R Package 'CDO', can be downloaded from https://sites.google.com/site/cdowithr/.

\section{Examples}

$\operatorname{frank}(0.3,10000, c(" 2007-10-23 "))$ 


\section{Description}

'gs' computes 5 tranches spreads under the Gaussian copula.

\section{Usage}

gs(rhoInput, MInput, dateInput)

\section{Arguments}

rhoInput a numeric giving the Pearson's correlation for dependence specification.

MInput a numeric giving the Monte Carlo simulation runs.

dateInput a character giving the pricing date, e.g. c("2007-10-23").

\section{Details}

Please make sure that the data sets of "defIntensity.csv" and "payday.csv" have been correctly installed in such paths: "C:/defIntensity.csv", "C:/payday.csv". The both data sets can be downloaded from "https://github.com/YafeiXu/xyfQuantlet".

\section{Value}

A vector with 5 numerics will be returned, from left to right: equity, junior mezzanine, senior mezzanine, junior senior, senior.

\section{Author(s)}

Yafei Xu <yafei.xu@hu-berlin.de>

\section{References}

The master thesis, CDO, HAME Copulas and an R Package 'CDO', can be downloaded from https://sites.google.com/site/cdowithr/.

\section{Examples}

$\operatorname{gs}(0.3,100, c(" 2007-10-23 "))$ 


\section{Description}

'gs1' computes 5 tranches spreads under the C_gs1 Model.

\section{Usage}

gs1 (theta1 Input, theta2Input, M, dateInput)

\section{Arguments}

\begin{tabular}{|c|c|}
\hline theta1 Input & a numeric giving the Pearson's correlation for dependence specification. \\
\hline theta2Input & a numeric giving the Pearson's correlation for dependence specification. \\
\hline MInput & a numeric giving the Monte Carlo simulation runs. \\
\hline dateInput & a character giving the pricing date, e.g. c("2007-10-23"). \\
\hline
\end{tabular}

\section{Details}

Please make sure that the data sets of "defIntensity.csv" and "payday.csv" have been correctly installed in such paths: "C:/defIntensity.csv", "C:/payday.csv". The both data sets can be downloaded from "https://github.com/YafeiXu/xyfQuantlet".

\section{Value}

A vector with 5 numerics will be returned, from left to right: equity, junior mezzanine, senior mezzanine, junior senior, senior.

\section{Author(s)}

Yafei Xu<yafei.xu@hu-berlin.de>

\section{References}

The master thesis, CDO, HAME Copulas and an R Package 'CDO', can be downloaded from https://sites.google.com/site/cdowithr/.

\section{Examples}

gs1 $(0.3,0.3,100, c(" 2007-10-23 "))$ 


\section{Description}

'gs2' computes 5 tranches spreads under the C_gs2 Model.

\section{Usage}

gs2(theta1 Input, M, dateInput)

\section{Arguments}
theta1 Input
a numeric giving the Pearson's correlation for dependence specification.
MInput a numeric giving the Monte Carlo simulation runs.
dateInput a character giving the pricing date, e.g. c("2007-10-23").

\section{Details}

Please make sure that the data sets of "defIntensity.csv" and "payday.csv" have been correctly installed in such paths: "C:/defIntensity.csv", "C:/payday.csv". The both data sets can be downloaded from "https://github.com/YafeiXu/xyfQuantlet".

\section{Value}

A vector with 5 numerics will be returned, from left to right: equity, junior mezzanine, senior mezzanine, junior senior, senior.

\section{Author(s)}

Yafei Xu <yafei.xu@hu-berlin.de>

\section{References}

The master thesis, CDO, HAME Copulas and an R Package 'CDO', can be downloaded from https://sites.google.com/site/cdowithr/.

\section{Examples}

$\operatorname{gs} 2(0.3,100, c(" 2007-10-23 "))$ 


\section{Description}

'gs3' computes 5 tranches spreads under the C_gs3 Model.

\section{Usage}

gs3(theta1Input, theta2Input, M, dateInput)

\section{Arguments}
theta1Input a numeric giving the Pearson's correlation for dependence specification.
theta2Input a numeric giving the Pearson's correlation for dependence specification.
MInput a numeric giving the Monte Carlo simulation runs.
dateInput a character giving the pricing date, e.g. c("2007-10-23").

\section{Details}

Please make sure that the data sets of "defIntensity.csv" and "payday.csv" have been correctly installed in such paths: "C:/defIntensity.csv", "C:/payday.csv". The both data sets can be downloaded from "https://github.com/YafeiXu/xyfQuantlet".

\section{Value}

A vector with 5 numerics will be returned, from left to right: equity, junior mezzanine, senior mezzanine, junior senior, senior.

\section{Author(s)}

Yafei Xu<yafei.xu@hu-berlin.de>

\section{References}

The master thesis, CDO, HAME Copulas and an R Package 'CDO', can be downloaded from https://sites.google.com/site/cdowithr/.

\section{Examples}

$\operatorname{gs} 3(0.3,0.4,100, c(" 2007-10-23 "))$ 


\section{Description}

'gs4' computes 5 tranches spreads under the C_gs4 Model.

\section{Usage}

gs4(theta1Input, theta2Input, M, dateInput)

\section{Arguments}

$\begin{array}{ll}\text { theta1Input } & \text { a numeric giving the Pearson's correlation for dependence specification. } \\ \text { theta2Input } & \text { a numeric giving the Pearson's correlation for dependence specification. } \\ \text { MInput } & \text { a numeric giving the Monte Carlo simulation runs. } \\ \text { dateInput } & \text { a character giving the pricing date, e.g. c("2007-10-23"). }\end{array}$

\section{Details}

Please make sure that the data sets of "defIntensity.csv" and "payday.csv" have been correctly installed in such paths: "C:/defIntensity.csv", "C:/payday.csv". The both data sets can be downloaded from "https://github.com/YafeiXu/xyfQuantlet".

\section{Value}

A vector with 5 numerics will be returned, from left to right: equity, junior mezzanine, senior mezzanine, junior senior, senior.

\section{Author(s)}

Yafei Xu <yafei.xu@hu-berlin.de>

\section{References}

The master thesis, CDO, HAME Copulas and an R Package 'CDO', can be downloaded from https://sites.google.com/site/cdowithr/.

\section{Examples}

gs4 $(0.3,0.4,100, c(" 2007-10-23 "))$ 


\section{Description}

'gs5' computes 5 tranches spreads under the C_gs5 Model.

\section{Usage}

gs5(theta1Input, theta2Input, theta3Input, M, dateInput)

\section{Arguments}

$\begin{array}{ll}\text { theta1Input } & \text { a numeric giving the Pearson's correlation for dependence specification. } \\ \text { theta2Input } & \text { a numeric giving the Pearson's correlation for dependence specification. } \\ \text { theta3Input } & \text { a numeric giving the Pearson's correlation for dependence specification. } \\ \text { MInput } & \text { a numeric giving the Monte Carlo simulation runs. } \\ \text { dateInput } & \text { a character giving the pricing date, e.g. c("2007-10-23"). }\end{array}$

\section{Details}

Please make sure that the data sets of "defIntensity.csv" and "payday.csv" have been correctly installed in such paths: "C:/defIntensity.csv", "C:/payday.csv". The both data sets can be downloaded from "https://github.com/YafeiXu/xyfQuantlet".

\section{Value}

A vector with 5 numerics will be returned, from left to right: equity, junior mezzanine, senior mezzanine, junior senior, senior.

\section{Author(s)}

Yafei Xu<yafei.xu@hu-berlin.de>

\section{References}

The master thesis, CDO, HAME Copulas and an R Package 'CDO', can be downloaded from https://sites.google.com/site/cdowithr/.

\section{Examples}

$\operatorname{gs} 5(0.3,0.4,0.5,100, c(" 2007-10-23 "))$ 


\section{Description}

'gs6' computes 5 tranches spreads under the C_gs6 Model.

\section{Usage}

gs6(theta1Input, theta2Input, theta3Input, M, dateInput)

\section{Arguments}

$\begin{array}{ll}\text { theta1 Input } & \text { a numeric giving the Pearson's correlation for dependence specification. } \\ \text { theta2Input } & \text { a numeric giving the Pearson's correlation for dependence specification. } \\ \text { theta3Input } & \text { a numeric giving the Pearson's correlation for dependence specification. } \\ \text { MInput } & \text { a numeric giving the Monte Carlo simulation runs. } \\ \text { dateInput } & \text { a character giving the pricing date, e.g. c("2007-10-23") }\end{array}$

\section{Details}

Please make sure that the data sets of "defIntensity.csv" and "payday.csv" have been correctly installed in such paths: "C:/defIntensity.csv", "C:/payday.csv". The both data sets can be downloaded from "https://github.com/YafeiXu/xyfQuantlet".

\section{Value}

A vector with 5 numerics will be returned, from left to right: equity, junior mezzanine, senior mezzanine, junior senior, senior.

\section{Author(s)}

Yafei Xu<yafei.xu@hu-berlin.de>

\section{References}

The master thesis, CDO, HAME Copulas and an R Package 'CDO', can be downloaded from https://sites.google.com/site/cdowithr/.

\section{Examples}

gs6 $(0.3,0.4,0.5,100, c(" 2007-10-23 "))$ 
gumbel CDO Pricing with the Gumbel copula

\section{Description}

'gumbel' computes 5 tranches spreads under the Gumbel copula.

\section{Usage}

gumbel(rhoInput, MInput, dateInput)

\section{Arguments}

rhoInput a numeric giving the Kendall's tau for dependence specification.

MInput a numeric giving the Monte Carlo simulation runs.

dateInput a character giving the pricing date, e.g. c("2007-10-23").

\section{Details}

Please make sure that the data sets of "defIntensity.csv" and "payday.csv" have been correctly installed in such paths: "C:/defIntensity.csv", "C:/payday.csv". The both data sets can be downloaded from "https://github.com/YafeiXu/xyfQuantlet".

\section{Value}

A vector with 5 numerics will be returned, from left to right: equity, junior mezzanine, senior mezzanine, junior senior, senior.

\section{Author(s)}

Yafei Xu <yafei.xu@hu-berlin.de>

\section{References}

The master thesis, CDO, HAME Copulas and an R Package 'CDO', can be downloaded from https://sites.google.com/site/cdowithr/.

\section{Examples}

gumbel $\left(0.3,100, c\left(" 2007-10-23^{\prime \prime}\right)\right)$ 
joe $\quad$ CDO Pricing with the Joe copula

\section{Description}

'joe' computes 5 tranches spreads under the Joe copula.

\section{Usage}

joe(rhoInput, MInput, dateInput)

\section{Arguments}

rhoInput a numeric giving the Kendall's tau for dependence specification.

MInput a numeric giving the Monte Carlo simulation runs.

dateInput a character giving the pricing date, e.g. c("2007-10-23").

\section{Details}

Please make sure that the data sets of "defIntensity.csv" and "payday.csv" have been correctly installed in such paths: "C:/defIntensity.csv", "C:/payday.csv". The both data sets can be downloaded from "https://github.com/YafeiXu/xyfQuantlet".

\section{Value}

A vector with 5 numerics will be returned, from left to right: equity, junior mezzanine, senior mezzanine, junior senior, senior.

\section{Author(s)}

Yafei Xu <yafei.xu@hu-berlin.de>

\section{References}

The master thesis, CDO, HAME Copulas and an R Package 'CDO', can be downloaded from https://sites.google.com/site/cdowithr/.

\section{Examples}

joe $(0.3,100, c(" 2007-10-23 "))$ 


\section{Description}

'mcc' computes 5 tranches spreads under the mixed Clayton-Clayton copula.

Usage

mcc(theta1Input, theta2Input, weightInput, M, dateInput)

\section{Arguments}

$\begin{array}{ll}\text { theta1Input } & \text { a numeric giving the Kendall's tau for dependence specification. } \\ \text { theta2Input } & \text { a numeric giving the Kendall's tau for dependence specification. } \\ \text { weightInput } & \text { a numeric giving the weight for the first component copula. } \\ \text { MInput } & \text { a numeric giving the Monte Carlo simulation runs. } \\ \text { dateInput } & \text { a character giving the pricing date, e.g. c("2007-10-23"). }\end{array}$

\section{Details}

Please make sure that the data sets of "defIntensity.csv" and "payday.csv" have been correctly installed in such paths: "C:/defIntensity.csv", "C:/payday.csv". The both data sets can be downloaded from "https://github.com/YafeiXu/xyfQuantlet".

\section{Value}

A vector with 5 numerics will be returned, from left to right: equity, junior mezzanine, senior mezzanine, junior senior, senior.

\section{Author(s)}

Yafei Xu<yafei.xu@hu-berlin.de>

\section{References}

The master thesis, CDO, HAME Copulas and an R Package 'CDO', can be downloaded from https://sites.google.com/site/cdowithr/.

\section{Examples}

$\operatorname{mcc}(0.3,0.4,0.5,100, c(" 2007-10-23 "))$ 


\section{Description}

'mcc' computes 5 tranches spreads under the mixed Clayton-Gumbel copula.

Usage

mcg(theta1Input, theta2Input, weightInput, M, dateInput)

\section{Arguments}

theta1Input a numeric giving the Kendall's tau for dependence specification.

theta2Input a numeric giving the Kendall's tau for dependence specification.

weightInput a numeric giving the weight for the first component copula.

MInput a numeric giving the Monte Carlo simulation runs.

dateInput a character giving the pricing date, e.g. c("2007-10-23").

\section{Details}

Please make sure that the data sets of "defIntensity.csv" and "payday.csv" have been correctly installed in such paths: "C:/defIntensity.csv", "C:/payday.csv". The both data sets can be downloaded from "https://github.com/YafeiXu/xyfQuantlet".

\section{Value}

A vector with 5 numerics will be returned, from left to right: equity, junior mezzanine, senior mezzanine, junior senior, senior.

\section{Author(s)}

Yafei Xu<yafei.xu@hu-berlin.de>

\section{References}

The master thesis, CDO, HAME Copulas and an R Package 'CDO', can be downloaded from https://sites.google.com/site/cdowithr/.

\section{Examples}

$\operatorname{mcg}\left(0.3,0.4,0.5,100, c\left(" 2007-10-23^{\prime \prime}\right)\right)$ 


\section{Description}

'mcj' computes 5 tranches spreads under the mixed Clayton-Joe copula.

Usage

mcj(theta1Input, theta2Input, weightInput, M, dateInput)

\section{Arguments}

$\begin{array}{ll}\text { theta1Input } & \text { a numeric giving the Kendall's tau for dependence specification. } \\ \text { theta2Input } & \text { a numeric giving the Kendall's tau for dependence specification. } \\ \text { weightInput } & \text { a numeric giving the weight for the first component copula. } \\ \text { MInput } & \text { a numeric giving the Monte Carlo simulation runs. } \\ \text { dateInput } & \text { a character giving the pricing date, e.g. c("2007-10-23"). }\end{array}$

\section{Details}

Please make sure that the data sets of "defIntensity.csv" and "payday.csv" have been correctly installed in such paths: "C:/defIntensity.csv", "C:/payday.csv". The both data sets can be downloaded from "https://github.com/YafeiXu/xyfQuantlet".

\section{Value}

A vector with 5 numerics will be returned, from left to right: equity, junior mezzanine, senior mezzanine, junior senior, senior.

\section{Author(s)}

Yafei Xu<yafei.xu@hu-berlin.de>

\section{References}

The master thesis, CDO, HAME Copulas and an R Package 'CDO', can be downloaded from https://sites.google.com/site/cdowithr/.

\section{Examples}

$\operatorname{mcj}\left(0.3,0.4,0.5,100, c\left(" 2007-10-23^{\prime \prime}\right)\right)$ 


\section{Description}

'mfc' computes 5 tranches spreads under the mixed Frank-Clayton copula.

Usage

mfc(theta1Input, theta2Input, weightInput, M, dateInput)

\section{Arguments}

theta1Input a numeric giving the Kendall's tau for dependence specification.

theta2Input a numeric giving the Kendall's tau for dependence specification.

weightInput a numeric giving the weight for the first component copula.

MInput a numeric giving the Monte Carlo simulation runs.

dateInput a character giving the pricing date, e.g. c("2007-10-23").

\section{Details}

Please make sure that the data sets of "defIntensity.csv" and "payday.csv" have been correctly installed in such paths: "C:/defIntensity.csv", "C:/payday.csv". The both data sets can be downloaded from "https://github.com/YafeiXu/xyfQuantlet".

\section{Value}

A vector with 5 numerics will be returned, from left to right: equity, junior mezzanine, senior mezzanine, junior senior, senior.

\section{Author(s)}

Yafei Xu<yafei.xu@hu-berlin.de>

\section{References}

The master thesis, CDO, HAME Copulas and an R Package 'CDO', can be downloaded from https://sites.google.com/site/cdowithr/.

\section{Examples}

$\operatorname{mfc}(0.3,0.4,0.5,100, c(" 2007-10-23 "))$ 


\section{Description}

'mff' computes 5 tranches spreads under the mixed Frank-Frank copula.

Usage

$\mathrm{mff}$ (theta1Input, theta2Input, weightInput, M, dateInput)

\section{Arguments}

$\begin{array}{ll}\text { theta1Input } & \text { a numeric giving the Kendall's tau for dependence specification. } \\ \text { theta2Input } & \text { a numeric giving the Kendall's tau for dependence specification. } \\ \text { weightInput } & \text { a numeric giving the weight for the first component copula. } \\ \text { MInput } & \text { a numeric giving the Monte Carlo simulation runs. } \\ \text { dateInput } & \text { a character giving the pricing date, e.g. c("2007-10-23"). }\end{array}$

\section{Details}

Please make sure that the data sets of "defIntensity.csv" and "payday.csv" have been correctly installed in such paths: "C:/defIntensity.csv", "C:/payday.csv". The both data sets can be downloaded from "https://github.com/YafeiXu/xyfQuantlet".

\section{Value}

A vector with 5 numerics will be returned, from left to right: equity, junior mezzanine, senior mezzanine, junior senior, senior.

\section{Author(s)}

Yafei Xu<yafei.xu@hu-berlin.de>

\section{References}

The master thesis, CDO, HAME Copulas and an R Package 'CDO', can be downloaded from https://sites.google.com/site/cdowithr/.

\section{Examples}

$\operatorname{mfc}\left(0.3,0.4,0.5,100, c\left(" 2007-10-23^{\prime \prime}\right)\right)$ 


\section{Description}

'mfg' computes 5 tranches spreads under the mixed Frank-Gumbel copula.

\section{Usage}

$\mathrm{mfg}$ (theta1Input, theta2Input, weightInput, M, dateInput)

\section{Arguments}

$\begin{array}{ll}\text { theta1Input } & \text { a numeric giving the Kendall's tau for dependence specification. } \\ \text { theta2Input } & \text { a numeric giving the Kendall's tau for dependence specification. } \\ \text { weightInput } & \text { a numeric giving the weight for the first component copula. } \\ \text { MInput } & \text { a numeric giving the Monte Carlo simulation runs. } \\ \text { dateInput } & \text { a character giving the pricing date, e.g. c("2007-10-23"). }\end{array}$

\section{Details}

Please make sure that the data sets of "defIntensity.csv" and "payday.csv" have been correctly installed in such paths: "C:/defIntensity.csv", "C:/payday.csv". The both data sets can be downloaded from "https://github.com/YafeiXu/xyfQuantlet".

\section{Value}

A vector with 5 numerics will be returned, from left to right: equity, junior mezzanine, senior mezzanine, junior senior, senior.

\section{Author(s)}

Yafei Xu<yafei.xu@hu-berlin.de>

\section{References}

The master thesis, CDO, HAME Copulas and an R Package 'CDO', can be downloaded from https://sites.google.com/site/cdowithr/.

\section{Examples}

$\operatorname{mfg}(0.3,0.4,0.5,100, c(" 2007-10-23 "))$ 


\section{Description}

'mfj' computes 5 tranches spreads under the mixed Frank-Joe copula.

Usage

mf $j$ (theta1Input, theta2Input, weightInput, M, dateInput)

\section{Arguments}

$\begin{array}{ll}\text { theta1Input } & \text { a numeric giving the Kendall's tau for dependence specification. } \\ \text { theta2Input } & \text { a numeric giving the Kendall's tau for dependence specification. } \\ \text { weightInput } & \text { a numeric giving the weight for the first component copula. } \\ \text { MInput } & \text { a numeric giving the Monte Carlo simulation runs. } \\ \text { dateInput } & \text { a character giving the pricing date, e.g. c("2007-10-23"). }\end{array}$

\section{Details}

Please make sure that the data sets of "defIntensity.csv" and "payday.csv" have been correctly installed in such paths: "C:/defIntensity.csv", "C:/payday.csv". The both data sets can be downloaded from "https://github.com/YafeiXu/xyfQuantlet".

\section{Value}

A vector with 5 numerics will be returned, from left to right: equity, junior mezzanine, senior mezzanine, junior senior, senior.

\section{Author(s)}

Yafei Xu <yafei.xu@hu-berlin.de>

\section{References}

The master thesis, CDO, HAME Copulas and an R Package 'CDO', can be downloaded from https://sites.google.com/site/cdowithr/.

\section{Examples}

$\operatorname{mfj}\left(0.3,0.4,0.5,100, c\left(" 2007-10-23^{\prime \prime}\right)\right)$ 


\section{Description}

'mgg' computes 5 tranches spreads under the mixed Gumbel-Gumbel copula.

Usage

mgg(theta1Input, theta2Input, weightInput, M, dateInput)

\section{Arguments}

theta1Input a numeric giving the Kendall's tau for dependence specification.

theta2Input a numeric giving the Kendall's tau for dependence specification.

weightInput a numeric giving the weight for the first component copula.

MInput a numeric giving the Monte Carlo simulation runs.

dateInput a character giving the pricing date, e.g. c("2007-10-23").

\section{Details}

Please make sure that the data sets of "defIntensity.csv" and "payday.csv" have been correctly installed in such paths: "C:/defIntensity.csv", "C:/payday.csv". The both data sets can be downloaded from "https://github.com/YafeiXu/xyfQuantlet".

\section{Value}

A vector with 5 numerics will be returned, from left to right: equity, junior mezzanine, senior mezzanine, junior senior, senior.

\section{Author(s)}

Yafei Xu <yafei.xu@hu-berlin.de>

\section{References}

The master thesis, CDO, HAME Copulas and an R Package 'CDO', can be downloaded from https://sites.google.com/site/cdowithr/.

\section{Examples}

$\operatorname{mgg}\left(0.3,0.4,0.5,100, c\left(" 2007-10-23^{\prime \prime}\right)\right)$ 


\section{Description}

'mgj' computes 5 tranches spreads under the mixed Gumbel-Joe copula.

\section{Usage}

mgj(theta1Input, theta2Input, weightInput, M, dateInput)

\section{Arguments}

$\begin{array}{ll}\text { theta1Input } & \text { a numeric giving the Kendall's tau for dependence specification. } \\ \text { theta2Input } & \text { a numeric giving the Kendall's tau for dependence specification. } \\ \text { weightInput } & \text { a numeric giving the weight for the first component copula. } \\ \text { MInput } & \text { a numeric giving the Monte Carlo simulation runs. } \\ \text { dateInput } & \text { a character giving the pricing date, e.g. c("2007-10-23"). }\end{array}$

\section{Details}

Please make sure that the data sets of "defIntensity.csv" and "payday.csv" have been correctly installed in such paths: "C:/defIntensity.csv", "C:/payday.csv". The both data sets can be downloaded from "https://github.com/YafeiXu/xyfQuantlet".

\section{Value}

A vector with 5 numerics will be returned, from left to right: equity, junior mezzanine, senior mezzanine, junior senior, senior.

\section{Author(s)}

Yafei Xu<yafei.xu@hu-berlin.de>

\section{References}

The master thesis, CDO, HAME Copulas and an R Package 'CDO', can be downloaded from https://sites.google.com/site/cdowithr/.

\section{Examples}

$\operatorname{mgj}\left(0.3,0.4,0.5,100, c\left(" 2007-10-23^{\prime \prime}\right)\right)$ 


\section{Description}

'mgsc' computes 5 tranches spreads under the mixed Gaussian-Clayton copula.

\section{Usage}

mgsc(theta1Input, theta2Input, weightInput, M, dateInput)

\section{Arguments}

$\begin{array}{ll}\text { theta1Input } & \text { a numeric giving the Pearson's correlation for dependence specification. } \\ \text { theta2Input } & \text { a numeric giving the Kendall's tau for dependence specification. } \\ \text { weightInput } & \text { a numeric giving the weight for the first component copula. } \\ \text { MInput } & \text { a numeric giving the Monte Carlo simulation runs. } \\ \text { dateInput } & \text { a character giving the pricing date, e.g. c("2007-10-23"). }\end{array}$

\section{Details}

Please make sure that the data sets of "defIntensity.csv" and "payday.csv" have been correctly installed in such paths: "C:/defIntensity.csv", "C:/payday.csv". The both data sets can be downloaded from "https://github.com/YafeiXu/xyfQuantlet".

\section{Value}

A vector with 5 numerics will be returned, from left to right: equity, junior mezzanine, senior mezzanine, junior senior, senior.

\section{Author(s)}

Yafei Xu<yafei.xu@hu-berlin.de>

\section{References}

The master thesis, CDO, HAME Copulas and an R Package 'CDO', can be downloaded from https://sites.google.com/site/cdowithr/.

\section{Examples}

$\operatorname{mgsc}\left(0.3,0.4,0.5,100, c\left(" 2007-10-23^{\prime \prime}\right)\right)$ 


\section{Description}

'mgsf' computes 5 tranches spreads under the mixed Gaussian-Frank copula.

Usage

mgsf(theta1Input, theta2Input, weightInput, M, dateInput)

\section{Arguments}

theta1Input a numeric giving the Pearson's correlation for dependence specification.

theta2Input a numeric giving the Kendall's tau for dependence specification.

weightInput a numeric giving the weight for the first component copula.

MInput a numeric giving the Monte Carlo simulation runs.

dateInput a character giving the pricing date, e.g. c("2007-10-23").

\section{Details}

Please make sure that the data sets of "defIntensity.csv" and "payday.csv" have been correctly installed in such paths: "C:/defIntensity.csv", "C:/payday.csv". The both data sets can be downloaded from "https://github.com/YafeiXu/xyfQuantlet".

\section{Value}

A vector with 5 numerics will be returned, from left to right: equity, junior mezzanine, senior mezzanine, junior senior, senior.

\section{Author(s)}

Yafei Xu<yafei.xu@hu-berlin.de>

\section{References}

The master thesis, CDO, HAME Copulas and an R Package 'CDO', can be downloaded from https://sites.google.com/site/cdowithr/.

\section{Examples}

$\operatorname{mgsf}(0.3,0.4,0.5,100, c(" 2007-10-23 "))$ 


\section{Description}

'mgsg' computes 5 tranches spreads under the mixed Gaussian-Gumbel copula.

\section{Usage}

mgsg(theta1Input, theta2Input, weightInput, M, dateInput)

\section{Arguments}

$\begin{array}{ll}\text { theta1Input } & \text { a numeric giving the Pearson's correlation for dependence specification. } \\ \text { theta2Input } & \text { a numeric giving the Kendall's tau for dependence specification. } \\ \text { weightInput } & \text { a numeric giving the weight for the first component copula. } \\ \text { MInput } & \text { a numeric giving the Monte Carlo simulation runs. } \\ \text { dateInput } & \text { a character giving the pricing date, e.g. c("2007-10-23"). }\end{array}$

\section{Details}

Please make sure that the data sets of "defIntensity.csv" and "payday.csv" have been correctly installed in such paths: "C:/defIntensity.csv", "C:/payday.csv". The both data sets can be downloaded from "https://github.com/YafeiXu/xyfQuantlet".

\section{Value}

A vector with 5 numerics will be returned, from left to right: equity, junior mezzanine, senior mezzanine, junior senior, senior.

\section{Author(s)}

Yafei Xu <yafei.xu@hu-berlin.de>

\section{References}

The master thesis, CDO, HAME Copulas and an R Package 'CDO', can be downloaded from https://sites.google.com/site/cdowithr/.

\section{Examples}

$\operatorname{mgsg}\left(0.3,0.4,0.5,100, c\left(" 2007-10-23^{\prime \prime}\right)\right)$ 
mgsgs CDO Pricing with the C_gsgs copula

\section{Description}

'mgsgs' computes 5 tranches spreads under the mixed Gaussian-Gaussian copula.

\section{Usage}

mgsgs(theta1Input, theta2Input, weightInput, M, dateInput)

\section{Arguments}

$\begin{array}{ll}\text { theta1Input } & \text { a numeric giving the Pearson's correlation for dependence specification. } \\ \text { theta2Input } & \text { a numeric giving the Pearson's correlation for dependence specification. } \\ \text { weightInput } & \text { a numeric giving the weight for the first component copula. } \\ \text { MInput } & \text { a numeric giving the Monte Carlo simulation runs. } \\ \text { dateInput } & \text { a character giving the pricing date, e.g. c("2007-10-23"). }\end{array}$

\section{Details}

Please make sure that the data sets of "defIntensity.csv" and "payday.csv" have been correctly installed in such paths: "C:/defIntensity.csv", "C:/payday.csv". The both data sets can be downloaded from "https://github.com/YafeiXu/xyfQuantlet".

\section{Value}

A vector with 5 numerics will be returned, from left to right: equity, junior mezzanine, senior mezzanine, junior senior, senior.

\section{Author(s)}

Yafei Xu<yafei.xu@hu-berlin.de>

\section{References}

The master thesis, CDO, HAME Copulas and an R Package 'CDO', can be downloaded from https://sites.google.com/site/cdowithr/.

\section{Examples}

$\operatorname{mgsgs}(0.3,0.4,0.5,100, c(" 2007-10-23 "))$ 


\section{Description}

'mgsj' computes 5 tranches spreads under the mixed Gaussian-Gaussian copula.

\section{Usage}

mgsj(theta1Input, theta2Input, weightInput, M, dateInput)

\section{Arguments}

$\begin{array}{ll}\text { theta1Input } & \text { a numeric giving the Pearson's correlation for dependence specification. } \\ \text { theta2Input } & \text { a numeric giving the Pearson's correlation for dependence specification. } \\ \text { weightInput } & \text { a numeric giving the weight for the first component copula. } \\ \text { MInput } & \text { a numeric giving the Monte Carlo simulation runs. } \\ \text { dateInput } & \text { a character giving the pricing date, e.g. c("2007-10-23"). }\end{array}$

\section{Details}

Please make sure that the data sets of "defIntensity.csv" and "payday.csv" have been correctly installed in such paths: "C:/defIntensity.csv", "C:/payday.csv". The both data sets can be downloaded from "https://github.com/YafeiXu/xyfQuantlet".

\section{Value}

A vector with 5 numerics will be returned, from left to right: equity, junior mezzanine, senior mezzanine, junior senior, senior.

\section{Author(s)}

Yafei Xu<yafei.xu@hu-berlin.de>

\section{References}

The master thesis, CDO, HAME Copulas and an R Package 'CDO', can be downloaded from https://sites.google.com/site/cdowithr/.

\section{Examples}

$\operatorname{mgsj}(0.3,0.4,0.5,100, c(" 2007-10-23 "))$ 


\section{Description}

'mgst' computes 5 tranches spreads under the mixed Gaussian-t copula.

Usage

mgst(theta1Input, theta2Input, weightInput, M, dateInput)

\section{Arguments}

$\begin{array}{ll}\text { theta1Input } & \text { a numeric giving the Pearson's correlation for dependence specification. } \\ \text { theta2Input } & \text { a numeric giving the Pearson's correlation for dependence specification. } \\ \text { weightInput } & \text { a numeric giving the weight for the first component copula. } \\ \text { MInput } & \text { a numeric giving the Monte Carlo simulation runs. } \\ \text { dateInput } & \text { a character giving the pricing date, e.g. c("2007-10-23"). }\end{array}$

\section{Details}

Please make sure that the data sets of "defIntensity.csv" and "payday.csv" have been correctly installed in such paths: "C:/defIntensity.csv", "C:/payday.csv". The both data sets can be downloaded from "https://github.com/YafeiXu/xyfQuantlet".

\section{Value}

A vector with 5 numerics will be returned, from left to right: equity, junior mezzanine, senior mezzanine, junior senior, senior.

\section{Author(s)}

Yafei Xu<yafei.xu@hu-berlin.de>

\section{References}

The master thesis, CDO, HAME Copulas and an R Package 'CDO', can be downloaded from https://sites.google.com/site/cdowithr/.

\section{Examples}

$\operatorname{mgst}(0.3,0.4,0.5,100, c(" 2007-10-23 "))$ 


\section{Description}

'mjj' computes 5 tranches spreads under the mixed Joe-Joe copula.

\section{Usage}

mjj(theta1Input, theta2Input, weightInput, M, dateInput)

\section{Arguments}

$\begin{array}{ll}\text { theta1Input } & \text { a numeric giving the Kendall's tau for dependence specification. } \\ \text { theta2Input } & \text { a numeric giving the Kendall's tau for dependence specification. } \\ \text { weightInput } & \text { a numeric giving the weight for the first component copula. } \\ \text { MInput } & \text { a numeric giving the Monte Carlo simulation runs. } \\ \text { dateInput } & \text { a character giving the pricing date, e.g. c("2007-10-23"). }\end{array}$

\section{Details}

Please make sure that the data sets of "defIntensity.csv" and "payday.csv" have been correctly installed in such paths: "C:/defIntensity.csv", "C:/payday.csv". The both data sets can be downloaded from "https://github.com/YafeiXu/xyfQuantlet".

\section{Value}

A vector with 5 numerics will be returned, from left to right: equity, junior mezzanine, senior mezzanine, junior senior, senior.

\section{Author(s)}

Yafei Xu<yafei.xu@hu-berlin.de>

\section{References}

The master thesis, CDO, HAME Copulas and an R Package 'CDO', can be downloaded from https://sites.google.com/site/cdowithr/.

\section{Examples}

$\operatorname{mjj}\left(0.3,0.4,0.5,100, c\left(" 2007-10-23^{\prime \prime}\right)\right)$ 


\section{Description}

'mtc' computes 5 tranches spreads under the mixed t-Clayton copula.

Usage

mtc(theta1Input, theta2Input, weightInput, M, dateInput)

\section{Arguments}

theta1Input a numeric giving the Pearson's correlation for dependence specification.

theta2Input a numeric giving the Kendall's tau for dependence specification.

weightInput a numeric giving the weight for the first component copula.

MInput a numeric giving the Monte Carlo simulation runs.

dateInput a character giving the pricing date, e.g. c("2007-10-23").

\section{Details}

Please make sure that the data sets of "defIntensity.csv" and "payday.csv" have been correctly installed in such paths: "C:/defIntensity.csv", "C:/payday.csv". The both data sets can be downloaded from "https://github.com/YafeiXu/xyfQuantlet".

\section{Value}

A vector with 5 numerics will be returned, from left to right: equity, junior mezzanine, senior mezzanine, junior senior, senior.

\section{Author(s)}

Yafei Xu<yafei.xu@hu-berlin.de>

\section{References}

The master thesis, CDO, HAME Copulas and an R Package 'CDO', can be downloaded from https://sites.google.com/site/cdowithr/.

\section{Examples}

$\operatorname{mtc}\left(0.3,0.4,0.5,100, c\left(" 2007-10-23^{\prime \prime}\right)\right)$ 


\section{Description}

'mtf' computes 5 tranches spreads under the mixed t-Frank copula.

Usage

$\mathrm{mtf}$ (theta1Input, theta2Input, weightInput, M, dateInput)

\section{Arguments}

theta1 Input a numeric giving the Pearson's correlation for dependence specification.

theta2Input a numeric giving the Kendall's tau for dependence specification.

weightInput a numeric giving the weight for the first component copula.

MInput a numeric giving the Monte Carlo simulation runs.

dateInput a character giving the pricing date, e.g. c("2007-10-23").

\section{Details}

Please make sure that the data sets of "defIntensity.csv" and "payday.csv" have been correctly installed in such paths: "C:/defIntensity.csv", "C:/payday.csv". The both data sets can be downloaded from "https://github.com/YafeiXu/xyfQuantlet".

\section{Value}

A vector with 5 numerics will be returned, from left to right: equity, junior mezzanine, senior mezzanine, junior senior, senior.

\section{$\operatorname{Author}(\mathbf{s})$}

Yafei Xu <yafei.xu@hu-berlin.de>

\section{References}

The master thesis, CDO, HAME Copulas and an R Package 'CDO', can be downloaded from https://sites.google.com/site/cdowithr/.

\section{Examples}

$\operatorname{mtf}(0.3,0.4,0.5,100, c(" 2007-10-23 "))$ 


\section{Description}

'mtg' computes 5 tranches spreads under the mixed t-Gumbel copula.

Usage

mtg(theta1Input, theta2Input, weightInput, M, dateInput)

\section{Arguments}

theta1Input a numeric giving the Pearson's correlation for dependence specification.

theta2Input a numeric giving the Kendall's tau for dependence specification.

weightInput a numeric giving the weight for the first component copula.

MInput a numeric giving the Monte Carlo simulation runs.

dateInput a character giving the pricing date, e.g. c("2007-10-23").

\section{Details}

Please make sure that the data sets of "defIntensity.csv" and "payday.csv" have been correctly installed in such paths: "C:/defIntensity.csv", "C:/payday.csv". The both data sets can be downloaded from "https://github.com/YafeiXu/xyfQuantlet".

\section{Value}

A vector with 5 numerics will be returned, from left to right: equity, junior mezzanine, senior mezzanine, junior senior, senior.

\section{Author(s)}

Yafei Xu<yafei.xu@hu-berlin.de>

\section{References}

The master thesis, CDO, HAME Copulas and an R Package 'CDO', can be downloaded from https://sites.google.com/site/cdowithr/.

\section{Examples}

$\operatorname{mtg}\left(0.3,0.4,0.5,100, c\left(" 2007-10-23^{\prime \prime}\right)\right)$ 


\section{Description}

'mtj' computes 5 tranches spreads under the mixed t-Joe copula.

Usage

mtj(theta1Input, theta2Input, weightInput, M, dateInput)

\section{Arguments}

theta1 Input a numeric giving the Pearson's correlation for dependence specification.

theta2Input a numeric giving the Kendall's tau for dependence specification.

weightInput a numeric giving the weight for the first component copula.

MInput a numeric giving the Monte Carlo simulation runs.

dateInput a character giving the pricing date, e.g. c("2007-10-23").

\section{Details}

Please make sure that the data sets of "defIntensity.csv" and "payday.csv" have been correctly installed in such paths: "C:/defIntensity.csv", "C:/payday.csv". The both data sets can be downloaded from "https://github.com/YafeiXu/xyfQuantlet".

\section{Value}

A vector with 5 numerics will be returned, from left to right: equity, junior mezzanine, senior mezzanine, junior senior, senior.

\section{Author(s)}

Yafei Xu<yafei.xu@hu-berlin.de>

\section{References}

The master thesis, CDO, HAME Copulas and an R Package 'CDO', can be downloaded from https://sites.google.com/site/cdowithr/.

\section{Examples}

$\operatorname{mtg}\left(0.3,0.4,0.5,100, c\left(" 2007-10-23^{\prime \prime}\right)\right)$ 


\section{Description}

'mtt' computes 5 tranches spreads under the mixed t-t copula.

Usage

mtt(theta1Input, theta2Input, weightInput, M, dateInput)

\section{Arguments}

$\begin{array}{ll}\text { theta1Input } & \text { a numeric giving the Pearson's correlation for dependence specification. } \\ \text { theta2Input } & \text { a numeric giving the Pearson's correlation for dependence specification. } \\ \text { weightInput } & \text { a numeric giving the weight for the first component copula. } \\ \text { MInput } & \text { a numeric giving the Monte Carlo simulation runs. } \\ \text { dateInput } & \text { a character giving the pricing date, e.g. c("2007-10-23"). }\end{array}$

\section{Details}

Please make sure that the data sets of "defIntensity.csv" and "payday.csv" have been correctly installed in such paths: "C:/defIntensity.csv", "C:/payday.csv". The both data sets can be downloaded from "https://github.com/YafeiXu/xyfQuantlet".

\section{Value}

A vector with 5 numerics will be returned, from left to right: equity, junior mezzanine, senior mezzanine, junior senior, senior.

\section{Author(s)}

Yafei Xu <yafei.xu@hu-berlin.de>

\section{References}

The master thesis, CDO, HAME Copulas and an R Package 'CDO', can be downloaded from https://sites.google.com/site/cdowithr/.

\section{Examples}

$\operatorname{mtt}(0.3,0.4,0.5,100, c(" 2007-10-23 "))$ 
ng13 CDO Pricing with the C_ng13 copula

\section{Description}

'ng13' computes 5 tranches spreads under the C_ng13 copula.

\section{Usage}

ng13(theta1Input, $M$, dateInput)

\section{Arguments}

$\begin{array}{ll}\text { theta1Input } & \text { a numeric giving the Kendall's tau for dependence specification. } \\ \text { MInput } & \text { a numeric giving the Monte Carlo simulation runs. } \\ \text { dateInput } & \text { a character giving the pricing date, e.g. c("2007-10-23"). }\end{array}$

\section{Details}

Please make sure that the data sets of "defIntensity.csv" and "payday.csv" have been correctly installed in such paths: "C:/defIntensity.csv", "C:/payday.csv". The both data sets can be downloaded from "https://github.com/YafeiXu/xyfQuantlet".

\section{Value}

A vector with 5 numerics will be returned, from left to right: equity, junior mezzanine, senior mezzanine, junior senior, senior.

\section{Author(s)}

Yafei Xu <yafei.xu@hu-berlin.de>

\section{References}

The master thesis, CDO, HAME Copulas and an R Package 'CDO', can be downloaded from https://sites.google.com/site/cdowithr/.

\section{Examples}

ng13(0.3, 100, c("2007-10-23")) 


\section{Description}

'ng 14 ' computes 5 tranches spreads under the C_ng14 copula.

\section{Usage}

ng14(theta1Input, theta2Input, M, dateInput)

\section{Arguments}

$\begin{array}{ll}\text { theta1 Input } & \text { a numeric giving the Kendall's tau for dependence specification. } \\ \text { theta2Input } & \text { a numeric giving the Kendall's tau for dependence specification. } \\ \text { MInput } & \text { a numeric giving the Monte Carlo simulation runs. } \\ \text { dateInput } & \text { a character giving the pricing date, e.g. c("2007-10-23"). }\end{array}$

\section{Details}

Please make sure that the data sets of "defIntensity.csv" and "payday.csv" have been correctly installed in such paths: "C:/defIntensity.csv", "C:/payday.csv". The both data sets can be downloaded from "https://github.com/YafeiXu/xyfQuantlet".

\section{Value}

A vector with 5 numerics will be returned, from left to right: equity, junior mezzanine, senior mezzanine, junior senior, senior.

\section{Author(s)}

Yafei Xu <yafei.xu@hu-berlin.de>

\section{References}

The master thesis, CDO, HAME Copulas and an R Package 'CDO', can be downloaded from https://sites.google.com/site/cdowithr/.

\section{Examples}

ng14(0.3,.5, 100, c("2007-10-23")) 
ng15 CDO Pricing with the C_ng15 copula

\section{Description}

'ng15' computes 5 tranches spreads under the C_ng15 copula.

\section{Usage}

ng15(theta1Input, theta2Input, M, dateInput)

\section{Arguments}

$\begin{array}{ll}\text { theta1Input } & \text { a numeric giving the Kendall's tau for dependence specification. } \\ \text { theta2Input } & \text { a numeric giving the Kendall's tau for dependence specification. } \\ \text { MInput } & \text { a numeric giving the Monte Carlo simulation runs. } \\ \text { dateInput } & \text { a character giving the pricing date, e.g. c("2007-10-23"). }\end{array}$

\section{Details}

Please make sure that the data sets of "defIntensity.csv" and "payday.csv" have been correctly installed in such paths: "C:/defIntensity.csv", "C:/payday.csv". The both data sets can be downloaded from "https://github.com/YafeiXu/xyfQuantlet".

\section{Value}

A vector with 5 numerics will be returned, from left to right: equity, junior mezzanine, senior mezzanine, junior senior, senior.

\section{Author(s)}

Yafei Xu <yafei.xu@hu-berlin.de>

\section{References}

The master thesis, CDO, HAME Copulas and an R Package 'CDO', can be downloaded from https://sites.google.com/site/cdowithr/.

\section{Examples}

ng15(0.3,.5, 100, c("2007-10-23")) 


\section{Description}

'ng16' computes 5 tranches spreads under the C_ng16 copula.

\section{Usage}

ng16(theta1Input, theta2Input, theta3Input, M, dateInput)

\section{Arguments}

$\begin{array}{ll}\text { theta1Input } & \text { a numeric giving the Kendall's tau for dependence specification. } \\ \text { theta2Input } & \text { a numeric giving the Kendall's tau for dependence specification. } \\ \text { theta3Input } & \text { a numeric giving the Kendall's tau for dependence specification. } \\ \text { MInput } & \text { a numeric giving the Monte Carlo simulation runs. } \\ \text { dateInput } & \text { a character giving the pricing date, e.g. c("2007-10-23"). }\end{array}$

\section{Details}

Please make sure that the data sets of "defIntensity.csv" and "payday.csv" have been correctly installed in such paths: "C:/defIntensity.csv", "C:/payday.csv". The both data sets can be downloaded from "https://github.com/YafeiXu/xyfQuantlet".

\section{Value}

A vector with 5 numerics will be returned, from left to right: equity, junior mezzanine, senior mezzanine, junior senior, senior.

\section{Author(s)}

Yafei Xu<yafei.xu@hu-berlin.de>

\section{References}

The master thesis, CDO, HAME Copulas and an R Package 'CDO', can be downloaded from https://sites.google.com/site/cdowithr/.

\section{Examples}

ng16(0.3,.5,.7, 100, c("2007-10-23")) 
stut CDO Pricing with the $C \_t$ copula

\section{Description}

'stut' computes 5 tranches spreads under the C_t copula.

\section{Usage}

stut (rhoInput, dF , MInput, dateInput)

\section{Arguments}

$\begin{array}{ll}\text { rhoInput } & \text { a numeric giving the Pearson's correlation for dependence specification. } \\ \text { dF } & \text { a numeric giving degree of freedom. } \\ \text { MInput } & \text { a numeric giving the Monte Carlo simulation runs. } \\ \text { dateInput } & \text { a character giving the pricing date, e.g. c("2007-10-23"). }\end{array}$

\section{Details}

Please make sure that the data sets of "defIntensity.csv" and "payday.csv" have been correctly installed in such paths: "C:/defIntensity.csv", "C:/payday.csv". The both data sets can be downloaded from "https://github.com/YafeiXu/xyfQuantlet".

\section{Value}

A vector with 5 numerics will be returned, from left to right: equity, junior mezzanine, senior mezzanine, junior senior, senior.

\section{Author(s)}

Yafei Xu<yafei.xu@hu-berlin.de>

\section{References}

The master thesis, CDO, HAME Copulas and an R Package 'CDO', can be downloaded from https://sites.google.com/site/cdowithr/.

\section{Examples}

$\operatorname{stut}(0.3,13,100, c(" 2007-10-23 "))$ 


\section{Description}

't38' computes 5 tranches spreads under the C_t38 copula.

\section{Usage}

t38(theta1Input, theta2Input, dF, MInput, dateInput)

\section{Arguments}

$\begin{array}{ll}\text { theta1Input } & \text { a numeric giving the Pearson's correlation for dependence specification. } \\ \text { theta2Input } & \text { a numeric giving the Pearson's correlation for dependence specification. } \\ \text { dF } & \text { a numeric giving the degree of freedom. } \\ \text { MInput } & \text { a numeric giving the Monte Carlo simulation runs. } \\ \text { dateInput } & \text { a character giving the pricing date, e.g. c("2007-10-23"). }\end{array}$

\section{Details}

Please make sure that the data sets of "defIntensity.csv" and "payday.csv" have been correctly installed in such paths: "C:/defIntensity.csv", "C:/payday.csv". The both data sets can be downloaded from "https://github.com/YafeiXu/xyfQuantlet".

\section{Value}

A vector with 5 numerics will be returned, from left to right: equity, junior mezzanine, senior mezzanine, junior senior, senior.

\section{Author(s)}

Yafei Xu<yafei.xu@hu-berlin.de>

\section{References}

The master thesis, CDO, HAME Copulas and an R Package 'CDO', can be downloaded from https://sites.google.com/site/cdowithr/.

\section{Examples}

$\mathrm{t} 38(0.3,0.3,13,100, c(" 2007-10-23 "))$ 


\section{Description}

't39' computes 5 tranches spreads under the $\mathrm{C}_{-} \mathrm{t} 39$ copula.

\section{Usage}

t39(theta1 Input, dF, M, dateInput)

\section{Arguments}
theta1Input a numeric giving the Pearson's correlation for dependence specification.
$\mathrm{dF}$ a numeric giving the degree of freedom.
M a numeric giving the Monte Carlo simulation runs.
dateInput a character giving the pricing date, e.g. c("2007-10-23").

\section{Details}

Please make sure that the data sets of "defIntensity.csv" and "payday.csv" have been correctly installed in such paths: "C:/defIntensity.csv", "C:/payday.csv". The both data sets can be downloaded from "https://github.com/YafeiXu/xyfQuantlet".

\section{Value}

A vector with 5 numerics will be returned, from left to right: equity, junior mezzanine, senior mezzanine, junior senior, senior.

\section{Author(s)}

Yafei Xu <yafei.xu@hu-berlin.de>

\section{References}

The master thesis, CDO, HAME Copulas and an R Package 'CDO', can be downloaded from https://sites.google.com/site/cdowithr/.

\section{Examples}

t39(0.3, 13, 100, c("2007-10-23")) 


\section{Description}

't40' computes 5 tranches spreads under the C_t40 copula.

\section{Usage}

t40(theta1Input, theta2Input, dF, M, dateInput)

\section{Arguments}

$\begin{array}{ll}\text { theta1Input } & \text { a numeric giving the Pearson's correlation for dependence specification. } \\ \text { theta2Input } & \text { a numeric giving the Pearson's correlation for dependence specification. } \\ \text { dF } & \text { a numeric giving the degree of freedom. } \\ \text { M } & \text { a numeric giving the Monte Carlo simulation runs. } \\ \text { dateInput } & \text { a character giving the pricing date, e.g. c("2007-10-23"). }\end{array}$

\section{Details}

Please make sure that the data sets of "defIntensity.csv" and "payday.csv" have been correctly installed in such paths: "C:/defIntensity.csv", "C:/payday.csv". The both data sets can be downloaded from "https://github.com/YafeiXu/xyfQuantlet".

\section{Value}

A vector with 5 numerics will be returned, from left to right: equity, junior mezzanine, senior mezzanine, junior senior, senior.

\section{Author(s)}

Yafei Xu<yafei.xu@hu-berlin.de>

\section{References}

The master thesis, CDO, HAME Copulas and an R Package 'CDO', can be downloaded from https://sites.google.com/site/cdowithr/.

\section{Examples}

$\mathrm{t} 40(0.3,0.3,13,100, \mathrm{c}(" 2007-10-23 "))$ 


\section{Description}

't41' computes 5 tranches spreads under the C_t41 copula.

\section{Usage}

t41(theta1Input, theta2Input, dF, M, dateInput)

\section{Arguments}

$\begin{array}{ll}\text { theta1Input } & \text { a numeric giving the Pearson's correlation for dependence specification. } \\ \text { theta2Input } & \text { a numeric giving the Pearson's correlation for dependence specification. } \\ \text { dF } & \text { a numeric giving the degree of freedom. } \\ \text { M } & \text { a numeric giving the Monte Carlo simulation runs. } \\ \text { dateInput } & \text { a character giving the pricing date, e.g. c("2007-10-23"). }\end{array}$

\section{Details}

Please make sure that the data sets of "defIntensity.csv" and "payday.csv" have been correctly installed in such paths: "C:/defIntensity.csv", "C:/payday.csv". The both data sets can be downloaded from "https://github.com/YafeiXu/xyfQuantlet".

\section{Value}

A vector with 5 numerics will be returned, from left to right: equity, junior mezzanine, senior mezzanine, junior senior, senior.

\section{Author(s)}

Yafei Xu<yafei.xu@hu-berlin.de>

\section{References}

The master thesis, CDO, HAME Copulas and an R Package 'CDO', can be downloaded from https://sites.google.com/site/cdowithr/.

\section{Examples}

t41(0.3,0.3, 13, 100, c("2007-10-23")) 


\section{Description}

't42' computes 5 tranches spreads under the $\mathrm{C}_{-} \mathrm{t} 42$ copula.

\section{Usage}

t42(theta1Input, theta2Input, theta3Input, dF, M, dateInput)

\section{Arguments}

theta1 Input a numeric giving the Pearson's correlation for dependence specification.

theta2Input a numeric giving the Pearson's correlation for dependence specification.

theta3Input a numeric giving the Pearson's correlation for dependence specification.

$\mathrm{dF} \quad$ a numeric giving the degree of freedom.

M a numeric giving the Monte Carlo simulation runs.

dateInput a character giving the pricing date, e.g. c("2007-10-23").

\section{Details}

Please make sure that the data sets of "defIntensity.csv" and "payday.csv" have been correctly installed in such paths: "C:/defIntensity.csv", "C:/payday.csv". The both data sets can be downloaded from "https://github.com/YafeiXu/xyfQuantlet".

\section{Value}

A vector with 5 numerics will be returned, from left to right: equity, junior mezzanine, senior mezzanine, junior senior, senior.

\section{Author(s)}

Yafei Xu <yafei.xu@hu-berlin.de>

\section{References}

The master thesis, CDO, HAME Copulas and an R Package 'CDO', can be downloaded from https://sites.google.com/site/cdowithr/.

\section{Examples}

$\mathrm{t} 42\left(0.3,0.3,0.3,13,100, c\left(" 2007-10-23^{\prime \prime}\right)\right)$ 


\section{Description}

't43' computes 5 tranches spreads under the $\mathrm{C}_{-} \mathrm{t} 42$ copula.

\section{Usage}

t43(theta1Input, theta2Input, theta3Input, $\mathrm{dF}, \mathrm{M}$, dateInput)

\section{Arguments}

$\begin{array}{ll}\text { theta1Input } & \text { a numeric giving the Pearson's correlation for dependence specification. } \\ \text { theta2Input } & \text { a numeric giving the Pearson's correlation for dependence specification. } \\ \text { theta3Input } & \text { a numeric giving the Pearson's correlation for dependence specification. } \\ \text { dF } & \text { a numeric giving the degree of freedom. } \\ \text { M } & \text { a numeric giving the Monte Carlo simulation runs. } \\ \text { dateInput } & \text { a character giving the pricing date, e.g. c("2007-10-23"). }\end{array}$

\section{Details}

Please make sure that the data sets of "defIntensity.csv" and "payday.csv" have been correctly installed in such paths: "C:/defIntensity.csv", "C:/payday.csv". The both data sets can be downloaded from "https://github.com/YafeiXu/xyfQuantlet".

\section{Value}

A vector with 5 numerics will be returned, from left to right: equity, junior mezzanine, senior mezzanine, junior senior, senior.

\section{Author(s)}

Yafei Xu <yafei.xu@hu-berlin.de>

\section{References}

The master thesis, CDO, HAME Copulas and an R Package 'CDO', can be downloaded from https://sites.google.com/site/cdowithr/.

\section{Examples}

$\mathrm{t} 43\left(0.3,0.3,0.3,13,100, \mathrm{c}\left(" 2007-10-23^{\prime \prime}\right)\right)$ 


\section{Index}

*Topic Archimedean Copula CDO-package, 2

*Topic CDO

CDO-package, 2

*Topic Credit Risk

CDO-package, 2

$*$ Topic Elliptical Copula

CDO-package, 2

*Topic Hierarchical Archimedean

Copula

CDO-package, 2

*Topic Mixed Copula

CDO-package, 2

*Topic copulas

CDO-package, 2

*Topic iTraxx Europe

CDO-package, 2

CDO (CDO-package), 2

CDO-package, 2

clayton, 3

frank, 4

gs, 5

gs 1,6

gs 2,7

gs 3,8

gs 4,9

gs 5,10

gs6, 11

gumbel, 12

joe, 13

mcc, 14

mcg, 15

$\mathrm{mcj}, 16$

$\mathrm{mfc}, 17$

$\mathrm{mff}, 18$

$\mathrm{mfg}, 19$

$\mathrm{mf}, 20$

mgg, 21

mgj, 22

mgsc, 23 mgsf, 24

mgsg, 25

mgsgs, 26

mgsj, 27

mgst, 28

$\mathrm{mjj}, 29$

mtc, 30

$\mathrm{mtf}, 31$

$\mathrm{mtg}, 32$

$\mathrm{mtj}, 33$

$\mathrm{mtt}, 34$

ng13, 35

ng14, 36

ng15, 37

ng16, 38

stut, 39

t 38,40

t 39,41

$\mathrm{t} 40,42$

$\mathrm{t} 41,43$

$\mathrm{t} 42,44$

t43, 45 